\title{
Doping a topological quantum spin liquid: slow holes in the Kitaev honeycomb model
}

\author{
Gábor B. Halász ${ }^{1}$, J. T. Chalker ${ }^{1}$, and R. Moessner ${ }^{2}$ \\ ${ }^{1}$ Theoretical Physics, Oxford University, 1 Keble Road, Oxford OX1 3NP, United Kingdom \\ ${ }^{2}$ Max-Planck-Institut für Physik komplexer Systeme, Nöthnitzer Straße 38, D-01187 Dresden, Germany
}

\begin{abstract}
We present a controlled microscopic study of mobile holes in the spatially anisotropic (Abelian) gapped phase of the Kitaev honeycomb model. We address the properties of (i) a single hole [its internal degrees of freedom as well as its hopping properties]; (ii) a pair of holes [their (relative) particle statistics and interactions]; (iii) the collective state for a finite density of holes. We find that each hole in the doped model has an eight-dimensional internal space, characterized by three internal quantum numbers: the first two "fractional" quantum numbers describe the binding to the hole of the fractional excitations (fluxes and fermions) of the undoped model, while the third "spin" quantum number determines the local magnetization around the hole. The fractional quantum numbers also encode fundamentally distinct particle properties, topologically robust against small local perturbations: some holes are free to hop in two dimensions, while others are confined to hop in one dimension only; distinct hole types have different particle statistics, and in particular, some of them exhibit non-trivial (anyonic) relative statistics. These particle properties in turn determine the physical properties of the multi-hole ground state at finite doping, and we identify two distinct ground states with different hole types that are stable for different model parameters. The respective hopping dimensionalities manifest themselves in an electrical conductivity approximately isotropic in one ground state and extremely anisotropic in the other one. We also compare our microscopic study with related mean-field treatments, and discuss the main discrepancies between the two approaches, which in particular involve the possibility of binding fractional excitations as well as the particle statistics of the holes. On a technical level, we describe the hopping of mobile holes via a quasi-stationary approach, where effective hopping matrix elements are calculated between ground states with stationary holes at different positions. This approach relies on the fact that the model remains exactly solvable in the presence of stationary holes, and that the motion of sufficiently slow holes does not generate bulk excitations in a gapped phase. When the bare hopping amplitude is much smaller than the energy gap, many of our results, in particular those on the hopping properties and the particle statistics, are exact.
\end{abstract}

\section{INTRODUCTION}

The behavior of a Mott insulator upon doping remains one of the constitutive open questions in the physics of stronglycorrelated electrons $\frac{1,2}{}$ Historically, this is in large part due to the identification of this issue $e^{2.3}$ as being central to the understanding of high-temperature superconductors $\stackrel{4}{\underline{4} \text { Indeed, it has }}$ been recognized that Mott insulators can enter a broad range of spin states, some of which are considerably more exotic than the familiar antiferromagnetic Néel state.$^{\frac{5}{}}$ In particular, Anderson suggested ${ }^{\underline{6}}$ that the parent state of high-temperature superconductors is a resonating-valence-bond (RVB) liquid state ${ }^{\underline{7}}$ with no conventional order. This suggestion in turn provided motivation for the study of such unconventional spin states, ${ }^{2.8}$ and it has been established ${ }^{9,10}$ that the RVB liquid state belongs to the class of fractional ${ }^{11}$ topological ${ }^{12}$ states. The effective low-energy excitations above these nonsymmetry-breaking topological states are fractional in the sense that they carry only a fraction of the spin and charge quantum numbers that characterize a single electron $\stackrel{11}{1}$ The simplest example of such low-energy fractionalization is spincharge separation in the case of the RVB liquid, where the elementary excitations are neutral spinful fermions (spinons) and charged spinless bosons (holons) ${ }^{\frac{9}{9}}$ For a doped topological state, it is then natural to ask how the hopping of an extra electron or a missing electron (hole) translates into the dynamics of these fractional excitations.

In this work, we provide a controlled and microscopic analysis of mobile holes hopping in a topological quantum spin liquid containing such fractional excitations. We are primar- ily interested in the internal degrees of freedom possessed by these holes, their manifestations in the single-particle behavior such as hopping properties and particle statistics, and their consequences for the multi-particle ground state that determines the observable physical properties. Our approach is complementary to previous phenomenological works on doped topological states as we study the exactly solvable Kitaev honeycomb model $\stackrel{13}{\underline{n}}$ This two-dimensional quantum spin model has a topological spin-liquid ground state with fractional excitations, $\frac{13}{\underline{3}}$ and it also remains exactly solvable in the

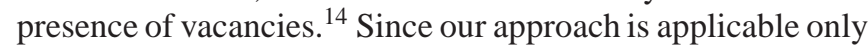
in the regime of slow hopping when the hopping amplitude is much smaller than the energy gap of the elementary excitations, we restrict our attention to the spatially anisotropic (Abelian) gapped phase of the model. For a recent numerical work on the spatially isotropic gapless phase, see the exactdiagonalization study by Trousselet et al.$\underline{15}$

There is an additional methodological interest in this work as the Kitaev honeycomb model lies at the intersection of an exact microscopic solution and a standard phenomenological treatment in terms of RVB trial wave functions $\frac{16}{6}$ that is applicable to doped Mott insulators in general. The trial wave function can optimize the magnetic interaction energy via (anti)ferromagnetic pairing, while a subsequent mean-field decomposition naturally leads to a BCS-type Hamiltonian. In the absence of doping, the constraint of single occupancy is enforced by an appropriate (numerical or approximate) projection procedure $\frac{16,17}{,}$ while in the presence of doping, this projection procedure requires softening.

In this framework, low-energy fractionalization in unconventional spin states is typically captured by a slave-particle 
(parton) construction, in which electrons are represented by combinations of fractional degrees of freedom such as spinons and holons. 18 Depending on the precise forms of the slaveparticle construction and the subsequent mean-field decomposition, several distinct slave-particle mean-field theories can be constructed for the same Hamiltonian. The possible meanfield saddle points are most efficiently classified in the framework of projective symmetry groups,,$\underline{18}$ while the fluctuations around these mean-field saddle points generally give rise to gauge theories..$^{18,19}$ Importantly, there are an extremely large number of distinct saddle points, 20 and it is hard to decide which of these saddle points are stable ${ }^{21}$ Given a Hamiltonian, it is not clear how to choose the most relevant saddle point, and therefore the construction of a slave-particle meanfield theory is not a fully controlled procedure.

The doped Kitaev honeycomb model has been studied extensively in the framework of slave-particle mean-field theories, ${ }^{22,23}$ and in particular, the mean-field construction by You et al. recovers the exact ground-state correlations in the limit of the undoped model ${ }^{23}$ Since the exact microscopic solution and the phenomenological mean-field construction coincide at this natural starting point of the investigation, the setting of the Kitaev honeycomb model provides a controlled way of clarifying the relation between the microscopic and the phenomenological approaches.

Our most important results about the properties of single mobile holes are summarized in Table \ In particular, we find that the holes in the doped model possess internal degrees of freedom because they can bind the fractional excitations of the undoped model. The holes therefore carry fractional quantum numbers, and these quantum numbers are robust against small local perturbations as they are associated with the superselection sectors of the model. Crucially, the distinct hole types with different quantum numbers have fundamentally different single-particle properties. Depending on their quantum numbers, holes can be either bosons or fermions, while holes with distinct quantum numbers can have non-trivial (anyonic) relative statistics. Furthermore, the various hole types have strikingly different hopping properties. Specifically, the hopping dimensionality is a function of the hole type: certain holes are free to hop in two dimensions, while others are confined to hop in one dimension only.

The internal degrees of freedom have a crucial effect on the physical properties of the doped model, and the fractional quantum numbers in the multi-particle ground state depend on the model parameters. This means that bare holes can be induced to bind fractional excitations in the ground state ${ }^{24}$ and that the presence of the resulting composite particles is observable in the physical properties. Importantly, our results can also be juxtaposed to those obtained from related slaveparticle mean-field theories. The most closely related meanfield treatment in Ref. 23 studies the isotropic gapless phase of the same model, and two significant observations arise from a careful comparison between the two approaches. First, the mean-field treatment unsurprisingly fails to capture the formation of composite particles consisting of bare holes and fractional excitations. Second, the particle statistics of bare holes are different in the two approaches: we find that they are fermions, while they are taken to be bosons by the slaveparticle construction of Ref. 23.

The structure of the paper is as follows. In Sec. I we provide an extended summary of our most important results. In Secs. III and IV we review the general properties of the undoped Kitaev honeycomb model and its spatially anisotropic gapped phase, respectively. In Sec. V] we introduce stationary holes into the model and specify their internal degrees of freedom. In Sec. VI we discuss the single-particle behavior of slow mobile holes, including their hopping properties and particle statistics. In Sec. VII, we describe the multi-particle ground state and the resulting physical properties of the doped model. In Sec. VIII, we qualitatively consider mobile holes beyond the regime of slow hopping. In Sec. IX we compare our exact microscopic results with the corresponding meanfield results in Ref. 23. Finally, in Sec. X] we conclude the paper with suggestions for future research.

\section{EXTENDED SUMMARY}

We now provide an extended summary of our most important results. The next two sections review the undoped Kitaev honeycomb model as background for the new results in the remaining sections. In Sec. III, we introduce the model and describe its exact solution. It is recalled that the ground state of the model has a topological degeneracy and that the elementary excitations above the ground state are fractional as they can only be created in pairs. There are two kinds of elementary excitations: fluxes, which always have a gapped energy spectrum, and fermions, which have a gapped or a gapless energy spectrum, depending on the model parameters. From Sec. IV] we restrict our attention to the gapped phase of the model, which is characterized by a gapped energy spectrum for both fluxes and fermions. We refer to a simple limiting point in this phase, the isolated dimer limit, where the model consists of infinitesimally coupled spin dimers. Furthermore, we explain the notion of superselection sectors to quantify the fractional nature of isolated excitation clusters.

In Sec. V, we introduce the formalism for describing holes, and discuss how the elementary degrees of freedom (modes) are affected by the presence of $n$ holes. The main result of this section is that each hole in the model has three localized internal modes at much smaller energies than the remaining bulk modes (fluxes and fermions). Excitations in these three internal modes are characterized by three internal quantum numbers: the flux quantum number $h=\{0,1\}$, the fermion quantum number $q=\{0,1\}$, and the plaquette quantum number $p=\{0,1\}$. The quantum numbers $h$ and $q$ specify the kinds of fractional excitations (fluxes and fermions) bound to the hole. They therefore determine its superselection sector via an equivalent excitation cluster (see Table \). The quantum number $p$ is related to the discrete spin-rotation symmetry $\sigma^{x, z} \rightarrow-\sigma^{x, z}$. It therefore acts as a spin quantum number and determines the local magnetization around the hole. Since $h$ and $q$ quantify the fractional nature of the hole, they are robust against arbitrary local perturbations of sufficiently small strength. This robustness does not extend to $p$ in general, but 


\begin{tabular}{c|c|c|c|c|c|c}
\hline \hline \multicolumn{2}{c|}{ Hole type } & Interpretation & Superselection sector & Hopping dimensionality & Absolute statistics & Relative statistics \\
\hline \multirow{2}{*}{$h=0$} & $q=0$ & Bare hole & Trivial $(1)$ & 2D (free \& isotropic) & Fermion & Trivial \\
& $q=1$ & Hole + fermion & Combined $(e \times m)$ & 2D (free \& anisotropic) & Boson & Non-trivial \\
\hline \multirow{2}{*}{$h=1$} & $q=0$ & Hole + flux & Electric $(e)$ & 1D (confined) & Fermion & Non-trivial \\
& $q=1$ & Hole + flux + fermion & Magnetic $(m)$ & 1D (confined) & Fermion & Non-trivial \\
\hline \hline
\end{tabular}

TABLE I: Summary of the most important hole properties for different combinations of the flux quantum number $h=\{0,1\}$ and the fermion quantum number $q=\{0,1\}$ : interpretations in terms of elementary excitations bound, superselection sectors of equivalent excitation clusters, generic hopping properties (see details in Fig. 8), absolute particle statistics, and relative particle statistics (see details in Table VII).

it does so in the important special case of a Heisenberg perturbation. We also consider interactions between holes and find an attractive two-hole interaction that is diagonal in $h$ and $p$ but not in $q$. To ensure that holes do not undergo pair formation or phase separation, we implicitly assume the presence of a sufficiently strong Coulomb repulsion as well.

In Sec. VI we introduce the formalism for describing hole hopping, and discuss the hopping properties of isolated holes in the model. Our approach is restricted to the regime of slow hopping, where the bulk modes are not excited as the hopping amplitude is much smaller than their energy gap. This section has two main results. First, the internal quantum numbers $h$, $q$, and $p$ are all conserved by the hopping. The various hole types with different quantum numbers can therefore be treated as distinct particles. Second, the hopping properties of a hole are unaffected by its quantum number $p$ but are strikingly affected by its quantum numbers $h$ and $q$. Since the model is spatially anisotropic in the gapped phase, the two perpendicular dimensions of the lattice are not equivalent. At a generic point of the gapped phase, $h=0$ holes are free to hop in two dimensions, while $h=1$ holes are confined to hop in one dimension only (see Table I). Restricting our attention to $h=0$ holes, the two-dimensional hopping problem of $q=0$ holes is approximately isotropic, while that of $q=1$ holes is strongly anisotropic. This difference is amplified in the isolated dimer limit, where $q=0$ holes remain free to hop in two dimensions, while $q=1$ holes become confined to hop in one dimension only. We also determine the absolute and the relative particle statistics of the various hole types (see Table ), and provide an intuitive explanation for our results by referring to the fermionic nature of the bare holes and the anyonic nature of the fractional excitations bound to them.

In Sec. VII we describe the multi-hole state representing a finite density of mobile holes, and determine the groundstate hole quantum numbers $h, q$, and $p$ that minimize the energy of such a multi-hole state. In the absence of hole interactions, there are two complementary regimes distinguished by the model parameters. In the first regime, all holes in the ground state are fermions with quantum numbers $h=0$ and $q=0$. They therefore fill two identical Fermi seas with different quantum numbers $p=\{0,1\}$. Since these holes are free to hop in two dimensions, the electrical conductivity is approximately isotropic. In the second regime, all holes in the ground state are fermions with quantum numbers $h=1$. They therefore fill four identical Fermi seas with different quantum numbers $q=\{0,1\}$ and $p=\{0,1\}$. Since these holes are confined to hop in one dimension only, the electrical conductivity is ex- tremely anisotropic. The two complementary regimes remain applicable in the presence of hole interactions as both the attractive interaction and the Coulomb repulsion are diagonal in the quantum number $h$. In the first regime, a mean-field treatment restricted to $h=0$ holes reveals that there is a critical hole density above which $q=1$ holes appear. Since these holes are bosons, their coherent condensation leads to charged superfluid behavior and a spontaneous net magnetization. In the second regime, a mean-field treatment restricted to $h=1$ holes reveals that scattering between coexisting $q=0$ holes and $q=1$ holes facilitates hopping in both dimensions of the lattice. This implies that the conductivity anisotropy becomes weaker as the hole density is increased.

In Sec.VIII, we qualitatively discuss hole hopping beyond the regime of slow hopping, where the bulk modes are excited as the hopping amplitude is larger than their energy gap. Each hole is then surrounded by a cloud of fluctuating excitations (fluxes and fermions), but the internal quantum numbers $h, q$, and $p$ are applicable as long as the hole density is sufficiently small so that the excitation clouds around different holes do not merge. However, any hole with quantum numbers other than $h=0$ and $q=0$ is unstable against a spontaneous decay into a lower-energy hole with $h=0$ and $q=0$.

In Sec. IX we compare our results from the exact description with those in Ref. 23 that are obtained from a mean-field treatment. By contrasting the respective ground states, we find two main discrepancies between the two approaches. First, the quantum numbers $h$ and $q$ that specify the kinds of fractional excitations bound to the hole are captured in the exact description but ignored in the mean-field treatment. Second, the two approaches predict different particle statistics for holes with $h=0$ and $q=0$ : they are fermions in the exact description but bosons in the mean-field treatment.

\section{KITAEV HONEYCOMB MODEL}

\section{A. Introduction of the model}

The Kitaev honeycomb model is an exactly solvable twodimensional quantum spin model 13 Each site of the underlying honeycomb lattice supports a spin one-half degree of freedom (particle), and each spin is coupled to its three neighbors by Ising interactions involving the three different spin components. The sites of the bipartite lattice can be divided into two sublattices $A$ and $B$, while the bonds can be divided into three 


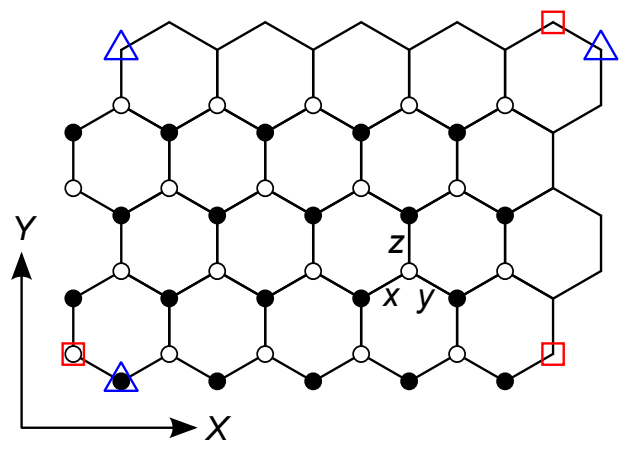

FIG. 1: (Color online) Illustration of the honeycomb lattice with dimensions $N_{X}=5$ and $N_{Y}=4$. Due to the periodic boundary conditions in the $X$ and $Y$ directions, several sites are identified with each other, such as the three sites marked by red rectangles and the three sites marked by blue triangles. Inequivalent sites in the sublattice $A(B)$ are marked by white (black) dots. Examples of the three bond types $(x, y, z)$ are also indicated.

classes $x, y$, and $z$ based on their orientations (see Fig. 1). If $\alpha_{l, l^{\prime}}=\{x, y, z\}$ gives the type of the bond connecting two neighboring sites $l$ and $l^{\prime}$, each site $l$ has three neighbors $\tilde{\alpha}(l)$ with $\tilde{\alpha}=\{x, y, z\}$ such that $\alpha_{l, \tilde{\alpha}(l)}=\tilde{\alpha}$. Using this notation, the Hamiltonian of the model reads as

$$
H_{\sigma}=-\sum_{l \in A} \sum_{\alpha=x, y, z} J_{\alpha} \sigma_{l}^{\alpha} \sigma_{\alpha(l)}^{\alpha},
$$

where $\sigma_{l}^{\alpha}$ are the physical (Pauli) spin operators, and $J_{x, y, z}$ are the Ising coupling strengths on the $x, y$, and $z$ bonds, respectively. In the following, we assume without loss of generality that $0 \leq J_{x} \leq J_{y} \leq J_{z}=1$.

We consider a lattice with periodic boundary conditions in both the horizontal $(X)$ and the vertical $(Y)$ directions. The $N_{X} \times N_{Y}$ lattice has $N \equiv N_{X} N_{Y}$ plaquettes, $2 N$ sites, and $3 N$ bonds (see Fig. 1). Based on their relative displacements in the $X$ direction, the horizontal plaquette stripes of the lattice can be divided into two classes, even and odd, such that an even (odd) stripe is neighbored only by odd (even) stripes. We assume that $N_{Y}$ is even so that periodic boundary conditions are applicable in the $Y$ direction without a stripe mismatch between the top and the bottom of the lattice. Note though that these boundary conditions are specified only for the purpose of completeness and that our main results are in fact independent of the boundary conditions.

\section{B. Flux degrees of freedom}

The Hamiltonian in Eq. (1) can be solved exactly by means of a standard procedure. 13 The first step is to notice that there is a commuting non-dynamic observable $W_{C}$ for each closed loop $C$ of the lattice. For a loop $C$ containing $L$ sites labeled $\{1,2, \ldots, L\}$, this non-dynamic observable is

$$
W_{C}=\sigma_{1}^{\alpha_{1,2}} \sigma_{2}^{\alpha_{1,2}} \sigma_{2}^{\alpha_{2,3}} \sigma_{3}^{\alpha_{2,3}} \ldots \sigma_{L}^{\alpha_{L, 1}} \sigma_{1}^{\alpha_{L, 1}}
$$

Since the lattice is bipartite, the length $L$ of the loop is always even. We also assume in the following that sites labeled with

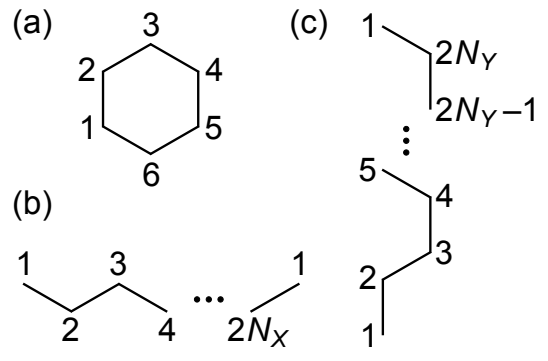

FIG. 2: Site labeling convention for the generators of the loop operator group: the plaquette operators $W_{P}$ (a) and the topological operators $W_{X}(\mathrm{~b})$ and $W_{Y}(\mathrm{c})$.

odd (even) numbers belong to the sublattice $A(B)$.

The loop operators $W_{C}$ are commuting non-dynamic observables because they commute with each other as well as the Hamiltonian $H_{\sigma}$. This means that the different flux sectors characterized by distinct eigenvalues $( \pm 1)$ of the loop operators can be considered independently. Furthermore, the group spanned by all loop operators is generated by a finite number of $\mathbb{Z}_{2}$ loop operators: those corresponding to the plaquettes $P$ and the topological strings $X$ and $Y$ going around the lattice in the $X$ and $Y$ directions. Using the site labeling convention in Fig. 2, these generating loop operators take the forms

$$
\begin{aligned}
& W_{P}=\sigma_{1}^{x} \sigma_{2}^{y} \sigma_{3}^{z} \sigma_{4}^{x} \sigma_{5}^{y} \sigma_{6}^{z} \\
& W_{X}=-\sigma_{1}^{z} \sigma_{2}^{z} \sigma_{3}^{z} \ldots \sigma_{2 N_{X}}^{z} \\
& W_{Y}=-\sigma_{1}^{x} \sigma_{2}^{y} \sigma_{3}^{y} \sigma_{4}^{x} \sigma_{5}^{x} \sigma_{6}^{y} \sigma_{7}^{y} \sigma_{8}^{x} \ldots \sigma_{2 N_{Y}-1}^{y} \sigma_{2 N_{Y}}^{x}
\end{aligned}
$$

Importantly, there are only $N-1$ independent plaquette operators due to the global constraint $\prod_{P} W_{P}=1$. This means that only $N+1$ flux degrees of freedom are found for the original $2 N$ spin degrees of freedom and that the remaining $N-1$ degrees of freedom still need to be identified. Note also that the excitation energies corresponding to the flux degrees of freedom are discussed in Secs. IIID and IV A

\section{Fermion degrees of freedom}

To solve the model exactly in each flux sector $\left\{W_{C}= \pm 1\right\}$, four Majorana fermions are introduced at each site $l$ of the lattice: $c_{l}$ and $b_{l}^{\alpha}$ with $\alpha=x, y, z \underline{13}^{13}$ The corresponding operators satisfy the standard anticommutation relations

$$
\begin{aligned}
\left\{b_{l}^{\alpha}, b_{l^{\prime}}^{\alpha^{\prime}}\right\} & =2 \delta_{l l^{\prime}} \delta_{\alpha \alpha^{\prime}}, & \left(b_{l}^{\alpha}\right)^{2}=1 \\
\left\{c_{l}, c_{l^{\prime}}\right\} & =2 \delta_{l l^{\prime}}, & c_{l}^{2}=1 \\
\left\{b_{l}^{\alpha}, c_{l^{\prime}}\right\} & =0 &
\end{aligned}
$$

The physical spin operators are then expressed in terms of the Majorana fermions as $\sigma_{l}^{\alpha}=i b_{l}^{\alpha} c_{l}$. From this expression and the relations in Eq. (4), certain properties of the spin operators can be immediately recovered: $\left[\sigma_{l}^{\alpha}, \sigma_{l^{\prime}}^{\alpha^{\prime}}\right]=0$ for $l \neq l^{\prime}$, $\left\{\sigma_{l}^{\alpha}, \sigma_{l}^{\alpha^{\prime}}\right\}=0$ for $\alpha \neq \alpha^{\prime}$, and $\left(\sigma_{l}^{\alpha}\right)^{2}=1$.

Since complex fermions are more straightforward to understand than Majorana fermions, it is useful to construct com- 
plex fermions by pairing up the Majorana fermions in an appropriate manner. Each Majorana fermion $b_{l}^{\alpha}$ belongs to an end of a bond, and the standard choice is to pair up the ones that belong to the two ends of the same bond. For each site $l \in A$, three complex bond fermions are then obtained as

$$
\chi_{l}^{\alpha}=\frac{1}{2}\left[b_{l}^{\alpha}-i b_{\alpha(l)}^{\alpha}\right], \quad\left(\chi_{l}^{\alpha}\right)^{\dagger}=\frac{1}{2}\left[b_{l}^{\alpha}+i b_{\alpha(l)}^{\alpha}\right] .
$$

Each Majorana fermion $c_{l}$ belongs to a site, and the standard choice is to pair up the ones that belong to any two sites connected by a $z$ bond. In terms of $c_{l, A} \equiv c_{l}$ and $c_{l, B} \equiv c_{z(l)}$ that are defined for each site $l \in A$, one complex matter fermion is then obtained for each pair of sites as

$$
f_{l}=\frac{1}{2}\left(c_{l, A}+i c_{l, B}\right), \quad f_{l}^{\dagger}=\frac{1}{2}\left(c_{l, A}-i c_{l, B}\right) .
$$

The state of the bond fermion $\chi_{l}^{\alpha}$ can be measured with the bond fermion operator $i b_{l}^{\alpha} b_{\alpha(l)}^{\alpha}=1-2\left(\chi_{l}^{\alpha}\right)^{\dagger} \chi_{l}^{\alpha}$, while the state of the matter fermion $f_{l}$ can be measured with the matter fermion operator $-i c_{l, A} c_{l, B}=1-2 f_{l}^{\dagger} f_{l}$. We say that a bond (matter) fermion is excited if its bond (matter) fermion operator takes an eigenvalue -1 rather than +1 .

When expressed in terms of the Majorana fermions, the Hamiltonian in Eq. (1) takes the form

$$
H_{\hat{u}}=i \sum_{l \in A} \sum_{\alpha=x, y, z} J_{\alpha} \hat{u}_{l, \alpha(l)} c_{l} c_{\alpha(l)},
$$

where the $3 N$ bond fermion operators $\hat{u}_{l, \alpha(l)} \equiv i b_{l}^{\alpha} b_{\alpha(l)}^{\alpha}$ are commuting non-dynamic observables because they commute with each other as well as the Hamiltonian $H_{\hat{u}}$. This means that the different bond fermion sectors characterized by distinct eigenvalues $( \pm 1)$ of the bond fermion operators can be considered independently. On the other hand, the Hamiltonian $H_{\hat{u}}$ is quadratic and hence exactly solvable in each bond fermion sector $\left\{u_{l, \alpha(l)} \equiv\left\langle\hat{u}_{l, \alpha(l)}\right\rangle= \pm 1\right\}$. If the Majorana fermions $c_{l}$ corresponding to the two sublattices are incorporated into two vectors $c_{A, B}$ with elements $\left(c_{A}\right)_{l}=c_{l, A}$ and $\left(c_{B}\right)_{l}=c_{l, B}$, the Hamiltonian in Eq. (7) becomes

$$
H_{u}=i c_{A}^{T} \cdot M \cdot c_{B}, \quad M_{l l^{\prime}}=u_{l, z\left(l^{\prime}\right)} J_{\alpha_{l, z\left(l^{\prime}\right)}},
$$

where $J_{\alpha_{l, z\left(l^{\prime}\right)}}=0$ if $l$ and $z\left(l^{\prime}\right)$ are not neighbors. The matrix $M$ has a singular value decomposition $M=U \cdot S \cdot V^{T}$, where $S$ is a positive-semidefinite diagonal matrix, while $U$ and $V$ are real orthogonal matrices. We assume in the following that the singular values $S_{k} \equiv S_{k k}$ are in an increasing order such that $0 \leq S_{1} \leq S_{2} \leq \ldots \leq S_{N}$. The orthogonal matrices $U$ and $V$ give a new set of Majorana fermions as

$$
\gamma_{k, A}=\sum_{l \in A} U_{l k} c_{l, A}, \quad \gamma_{k, B}=\sum_{l \in A} V_{l k} c_{l, B},
$$

and the corresponding complex matter fermions become

$$
\phi_{k}=\frac{1}{2}\left(\gamma_{k, A}+i \gamma_{k, B}\right), \phi_{k}^{\dagger}=\frac{1}{2}\left(\gamma_{k, A}-i \gamma_{k, B}\right) .
$$

In terms of these new matter fermions $\phi_{k}$, the Hamiltonian in Eq. (8) takes the free-fermion form

$$
H_{u}=\sum_{k=1}^{N} S_{k}\left(2 \phi_{k}^{\dagger} \phi_{k}-1\right) .
$$

The ground-state energy in the given bond fermion sector is then $-\sum_{k} S_{k}$, and the elementary excitations are the free matter fermions $\phi_{k}$ with excitation energies $2 S_{k}$.

It is important to understand the relation between the commuting non-dynamic observables in the physical spin picture and the Majorana fermion picture: the loop operators and the bond fermion operators, or equivalently, the flux sectors and the bond fermion sectors. When expressed in terms of the Majorana fermions, the loop operators $W_{C}$ take the form

$$
\begin{aligned}
W_{C} & =b_{1}^{\alpha_{1,2}} b_{2}^{\alpha_{1,2}} b_{2}^{\alpha_{2,3}} b_{3}^{\alpha_{2,3}} \ldots b_{L}^{\alpha_{L, 1}} b_{1}^{\alpha_{L, 1}} \\
& =\hat{u}_{1,2} \hat{u}_{3,2} \hat{u}_{3,4} \hat{u}_{5,4} \ldots \hat{u}_{L-1, L} \hat{u}_{1, L}
\end{aligned}
$$

and in particular, the plaquette operators $W_{P}$ become

$$
W_{P}=\hat{u}_{1,2} \hat{u}_{3,2} \hat{u}_{3,4} \hat{u}_{5,4} \hat{u}_{5,6} \hat{u}_{1,6} .
$$

These expressions show that the non-dynamic observables in the physical spin picture are uniquely determined by those in the Majorana fermion picture. However, the converse can not be true because there are $3 N$ bond fermion operators in the Majorana fermion picture for only $N+1$ loop operators in the physical spin picture. In fact, there is a gauge transformation $D_{l} \equiv b_{l}^{x} b_{l}^{y} b_{l}^{z} c_{l}$ for each site $l$ that flips three bond fermions but does not flip any loops. This means that the bond fermion sectors before and after the gauge transformation correspond to the same flux sector. Since $D \equiv \prod_{l} D_{l}$ does not flip any bond fermions, there are $2 N-1$ independent gauge transformations $D_{l}$, and the discrepancy between the numbers of non-dynamic observables is thus explained.

The gauge redundancy in the Majorana fermion picture follows from an enlarged Hilbert space with respect to the physical spin picture. In particular, the Hilbert space of a single site is 4 dimensional in the Majorana fermion picture and only 2 dimensional in the physical spin picture. This discrepancy is consistent with the fact that the spin identity $-i \sigma_{l}^{x} \sigma_{l}^{y} \sigma_{l}^{z} \equiv 1$ in the physical spin picture translates into the gauge constraint $D_{l}=+1$ in the Majorana fermion picture. In fact, all the states in the Majorana fermion picture that are related to each other by gauge transformations $D_{l}$ are equivalent descriptions of the same state in the physical spin picture. This physical state can be obtained from any of the gauge-related states by a projection onto the subspace with $D_{l}=+1$ for all $l$. The corresponding projection operator takes the form

$$
\mathcal{P}=\prod_{l}\left(\frac{1+D_{l}}{2}\right)=\mathcal{P}^{\prime}(1+D),
$$

where $\mathcal{P}^{\prime}$ contains all terms in $\mathcal{P}$ that flip bond fermions in inequivalent ways ${ }^{25}$ Since $D=(-1)^{N_{\chi}+N_{f}}$ when expressed in terms of the bond fermion number $N_{\chi} \equiv \sum_{\alpha} \sum_{l \in A}\left(\chi_{l}^{\alpha}\right)^{\dagger} \chi_{l}^{\alpha}$ and the matter fermion number $N_{f} \equiv \sum_{l \in A} f_{l}^{\dagger} f_{l}$, any states 
with odd total fermion number are projected to zero. There is a resulting global constraint for physical states: the total fermion number $N_{\chi}+N_{f}$ must be even. In each bond fermion sector with an even (odd) number of excited bond fermions, the number of excited matter fermions also must be even (odd). This means that only $N-1$ matter fermions can be excited independently from each other. The original $2 N$ spin degrees of freedom are then fully recovered via the identification of the $2 N$ natural degrees of freedom in the model: the $N+1$ fluxes and the $N-1$ fermions.

\section{Ground state and excitations}

The exact solution of the model provides a simple procedure for identifying its ground state 13 Each flux sector can be considered individually and represented with one of its corresponding bond fermion sectors. The ground state in the flux sector is then projected from that in the bond fermion sector (see Sec. IIIC), and the overall ground state is the lowest lying of all these individual ground states. Furthermore, it can be shown using translational invariance that the ground state is in the trivial flux sector: the one in which $W_{P}=+1$ for all plaquettes. ${ }^{26}$ The ground-state energy $\Gamma_{0}$ is then $-\sum_{k} S_{k}$ as obtained from the matrix $M$ in Eq. (8) using the trivial bond fermion sector: the one in which $u_{l, \alpha(l)}=+1$ for all bonds. Note that there are in principle four trivial flux sectors corresponding to the topological eigenvalues $W_{X, Y}= \pm 1$ and that this leads to the existence of four degenerate ground states. However, the topological degrees of freedom are impossible to excite locally. We therefore neglect them in the following by considering only the trivial topological sector with $W_{X}=W_{Y}=+1$. This means that the effective number of degrees of freedom is reduced to $2 N-2$.

It is also revealed by the exact solution that the elementary excitations above the ground state are plaquettes (fluxes) and fermions. ${ }^{13}$ We say that a plaquette $P$ is excited (carries a flux) if its plaquette operator $W_{P}$ takes an eigenvalue -1 rather than +1 . In the presence of flux excitations, the flux sector can no longer be represented with the trivial bond fermion sector, and the energy $-\sum_{k} S_{k}$ is larger than $\Gamma_{0}$. This difference translates into a finite flux excitation energy. Note that fluxes can only be excited pairwise due to the global constraint $\prod_{P} W_{P}=1$. The matter fermion excitations $\phi_{k}$ have excitation energies $E_{k} \equiv 2 S_{k}$, and by considering the distribution of these energies, two distinct phases of the model can be identified. In the gapless phase with $J_{z}<J_{x}+J_{y}$, the smallest excitation energies $E_{k}$ vanish in the thermodynamic limit. In the gapped phase with $J_{z}>J_{x}+J_{y}$, the excitation energies $E_{k}$ are all finite in the thermodynamic limit. Note that fermions can only be excited pairwise due to the global constraint that $N_{\chi}+N_{f}$ must be even.

\section{GAPPED PHASE OF THE MODEL}

In the following, we restrict our attention to the gapped phase of the Kitaev honeycomb model, where the coupling strengths satisfy $J_{x}+J_{y}<J_{z}$. Since all fluxes and fermions have finite excitation energies, the ground state in this phase is separated from the excited states by a finite energy gap. We measure all energies in units of the largest coupling strength $J_{z}=1$ and choose the two smaller coupling strengths $J_{x, y}$ to be equal. The model is then parameterized by the dimensionless coupling strength $J \equiv J_{x}=J_{y}<1 / 2$.

\section{A. Isolated dimer limit}

When considering the gapped phase, it is useful to start any discussion in the isolated dimer limit of $J=0$. In this limit, the model separates into $N$ isolated (non-interacting) spin dimers along $z$ bonds ${ }^{27}$ Since the two spins in any dimer are coupled by a ferromagnetic Ising term $-\sigma_{l}^{z} \sigma_{z(l)}^{z}$, they must be either both up or both down in the ground state. However, there is still an exponentially large ground-state degeneracy as each dimer can choose from two configurations. This degeneracy can then be lifted by applying a perturbation theory in the dimensionless coupling strength $J \ll 1.14$ At fourth order in $J$, the projection of the Hamiltonian in Eq. (1) onto the degenerate ground-state subspace is

$$
\tilde{H}_{\sigma}=-N-\tilde{C}(N, J)-\frac{J^{4}}{16} \sum_{P} W_{P} .
$$

The first term is the ground-state energy at $J=0$ and the remaining terms are the perturbative corrections: the constant term $\tilde{C}(N, J)$ shifts the energy of the entire subspace, while the last term lifts the ground-state degeneracy by specifying the flux sector. In accordance with Sec. IIID the actual ground state has $W_{P}=+1$ for all plaquettes.

It is instructive to write this ground state $|\Omega\rangle$ in terms of both the physical spins and the Majorana fermions. In the physical spin picture, it can be obtained by a projection from any state with $\sigma_{l}^{z} \sigma_{z(l)}^{z}=+1$ for all dimers onto the subspace with $W_{P}=+1$ for all plaquettes. For example, by projecting from the all-spins-up state $|\Uparrow\rangle$, the ground state becomes

$$
|\Omega\rangle=\prod_{P}\left(\frac{1+W_{P}}{2}\right)|\Uparrow\rangle .
$$

In the Majorana fermion picture, the trivial flux sector is represented with the trivial bond fermion sector, and the matrix $M$ in Eq. (8) is the unit matrix. Since the free matter fermions $\phi_{k}$ in Eq. (10) are then identical to the original matter fermions $f_{l}$ in Eq. (6), the ground state is the vacuum of the bond fermions and the original matter fermions. Formally, this vacuum state $|0\rangle$ is defined by $\chi_{l}^{\alpha}|0\rangle=0$ and $f_{l}|0\rangle=0$ for all $l$ and $\alpha$. The physical ground state in Eq. 16 is then $|\Omega\rangle=\mathcal{P}|0\rangle$.

The excitations above the ground state can be discussed in a similar manner. The flux excitations are obtained by projecting onto a subspace with excited plaquettes $W_{P}=-1$ in the physical spin picture and by exciting an appropriate set of bond fermions in the Majorana fermion picture. Due to the presence of the gauge transformations $D_{l}$, it is possible to represent any flux sector with a bond fermion sector in 
which only $x$ and $y$ bond fermions are excited. Since the matrix $M$ in Eq. (8) does not depend on these bond fermions for $J=0$, we recover the result in Eq. (15) that the flux excitation energies $E_{P} \sim J^{4}$ vanish when $J \rightarrow 0$. The fermion excitations are obtained by projecting from a state with broken dimers $\sigma_{l}^{z} \sigma_{z(l)}^{z}=-1$ in the physical spin picture and by exciting the corresponding matter fermions in the Majorana fermion picture. Since $u_{l, z(l)}=+1$ for all dimers when only $x$ and $y$ bond fermions are excited, the relation $\sigma_{l}^{z} \sigma_{z(l)}^{z}=\hat{u}_{l, z(l)}\left(1-2 f_{l}^{\dagger} f_{l}\right)$ shows that excited matter fermions indeed correspond to broken dimers. Furthermore, it follows from both pictures that these fermion excitations all have exactly the same energy $E_{f}=2$.

It is a conceptual problem that we require $J>0$ for a finite plaquette excitation energy but $J=0$ for the presence of the isolated dimers. In fact, since the localized matter fermions at $J=0$ all have the same excitation energy, even an infinitesimally small perturbation $J \ll 1$ is enough to delocalize them across the entire lattice and have them form a band of a small width $\Delta E_{f} \sim J$. This implies that the free (delocalized) matter fermions $\phi_{k}$ and the original (localized) matter fermions $f_{l}$ are entirely different for any $J>0$. To obtain the ground state at $J>0$, the vacuum state $|0\rangle$ is then projected onto the subspace where no free matter fermions $\phi_{k}$ are excited. Using this method, the physical ground state takes the form

$$
|\Omega\rangle=\mathcal{P} \prod_{k=1}^{N}\left(\phi_{k} \phi_{k}^{\dagger}\right)|0\rangle .
$$

Although the perturbation mixes the various creation operators, and consequently, the various annihilation operators together, it does not significantly mix the creation operators with the annihilation operators. This implies that the $J>0$ ground state in Eq. (17) is close to the $J=0$ ground state $\mathcal{P}|0\rangle$ and can be described faithfully in terms of the localized matter fermions. In the following, we therefore often simultaneously assume a finite plaquette excitation energy and localized matter fermions, always mentioning when the perturbative interactions between the matter fermions are important.

\section{B. Global constraints and superselection sectors}

The numbers of independent flux and fermion excitations are limited by two essential global constraints. In the physical spin picture, these two constraints can be obtained by noticing that the product of all plaquette operators $W_{P}$ corresponding to plaquettes in even $(\eta)$ stripes, or alternatively, plaquettes in odd $(\mu)$ stripes is equivalent to the product of all dimer operators $\lambda_{l} \equiv \sigma_{l}^{z} \sigma_{z(l)}^{z}$. Mathematically, these two relations are

$$
\prod_{P \in \eta} W_{P}=\prod_{P \in \mu} W_{P}=\prod_{l \in A} \lambda_{l} .
$$

Since the $W_{P}$ and the $\lambda_{l}$ are all $\mathbb{Z}_{2}$ variables, the first equality recovers the global constraint $\prod_{P} W_{P}=1$, while the second equality becomes $\prod_{l \in A} \lambda_{l} \prod_{P \in \mu} W_{P}=1$. In the Majorana fermion picture, the first equality is automatically satisfied because $\hat{u}_{l, \alpha(l)}^{2}=1$ for all bonds. The second equality can be understood by noticing that an excited $z$ bond fermion corresponds to two excited plaquettes that are either both in an even stripe or both in an odd stripe while an excited $x$ or $y$ bond fermion corresponds to one excited plaquette in an even stripe and one excited plaquette in an odd stripe. Since this property translates into $\prod_{P \in \mu} W_{P}=(-1)^{N_{\chi^{x}}+N_{\chi^{y}}}$ and the relation $\lambda_{l}=\hat{u}_{l, z(l)}\left(1-2 f_{l}^{\dagger} f_{l}\right)$ implies $\prod_{l \in A} \lambda_{l}=(-1)^{N_{\chi^{z}}+N_{f}}$, the second equality recovers the global constraint that $N_{\chi}+N_{f}$ must be even for physical states.

There is an alternative formulation of the global constraints given in Eq. (18) where one electric (magnetic) charge $e(m)$ is assigned to each excited plaquette in an even (odd) stripe and one from both charges $e$ and $m$ is assigned to each broken dimer. The global constraints in this formulation are that the total numbers of electric charges $\left(N_{e}\right)$ and magnetic charges $\left(N_{m}\right)$ both must be even. $\frac{13}{}$ In particular, if there are isolated clusters of excitations in the lattice, each of them can be classified into four superselection sectors based on the types of unpaired charges it contains: trivial (1), electric $(e)$, magnetic $(m)$, and combined $(\varepsilon \equiv e \times m)$. When different clusters are combined, the superselection sector of the combined cluster is given by the fusion rules in Table [I Using this language, the global constraints mean that the combination of all clusters belongs to the trivial superselection sector 1 .

\begin{tabular}{c|cccc}
\hline \hline & 1 & $e$ & $m$ & $\varepsilon$ \\
\hline 1 & 1 & $e$ & $m$ & $\varepsilon$ \\
$e$ & $e$ & 1 & $\varepsilon$ & $m$ \\
$m$ & $m$ & $\varepsilon$ & 1 & $e$ \\
$\varepsilon$ & $\varepsilon$ & $m$ & $e$ & 1 \\
\hline \hline
\end{tabular}

TABLE II: Fusion rules governing the combination of superselection sectors when different excitation clusters are combined.

The most important property of the superselection sectors is that they are robust against arbitrary local perturbations. Since a local perturbation acts only within one excitation cluster, it could only change the superselection sector of the cluster by also violating at least one global constraint. The superselection sector of an excitation cluster can then only be changed by a non-local perturbation that also changes the superselection sector of a different cluster or creates an additional cluster with a non-trivial superselection sector.

\section{STATIONARY HOLES}

\section{A. Description of holes}

We introduce $n$ holes into the Kitaev honeycomb model by removing the spin one-half particles from $n$ sites of the honeycomb lattice. For the model with $n>0$ holes, the exact solution in Sec. [III is still applicable, but it needs to be performed in a different way because there are no Majorana fermions

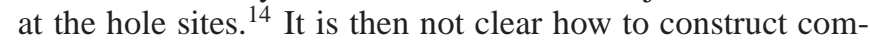


plex fermions from the remaining Majorana fermions, and the bond fermion operators, or equivalently, the plaquette operators acting on the hole sites become ill-defined.

To fix this problem, we use an alternative description: the spin one-half particles are not actually removed from the hole sites, but only their Ising interactions with their neighbors are switched off. This way, we obtain $2^{n}$ copies of the original model that correspond to the different configurations of the $n$ non-interacting hole spins. Since there are still Majorana fermions at all sites, the bond fermion operators and the plaquette operators remain well-defined. This means that the exact solution can be performed in exactly the same way as in Sec. III However, there is an additional $2^{n}$-fold degeneracy due to the presence of the non-interacting hole spins, which is unphysical and hence must be discarded.

Formally, we can demand all hole spins to be in the spinup state: $\sigma_{l}^{z}=+1$ for all sites $l \in \Delta$, where $\Delta$ is the set of hole sites. To obtain a physical state, we then need to use the appropriate projection operator, which takes the form

$$
\mathcal{Q}_{\Delta}=\prod_{l \in \Delta}\left(\frac{1+\sigma_{l}^{z}}{2}\right)=\prod_{l \in \Delta}\left(\frac{1+i b_{l}^{z} c_{l}}{2}\right) .
$$

Note that the treatment of the unphysical hole spins is completely analogous to the treatment of the unphysical Majorana fermions. In the Majorana fermion picture, different states corresponding to the same state in the physical spin picture are related by gauge transformations $D_{l}$. We can work in different gauges and then use the projector $\mathcal{P}$ to enforce the constraint $D_{l}=+1$ at all sites. In the hole spin picture, different states corresponding to the same state in the actual hole picture are related by gauge transformations $\sigma_{l \in \Delta}^{z}$. We can work in different gauges and then use the projector $\mathcal{Q}_{\Delta}$ to enforce the constraint $\sigma_{l}^{z}=+1$ at all hole sites.

\section{B. Internal degrees of freedom}

We now investigate how the excitations above the ground state as discussed in Sec. IIID are affected by the introduction of $n>0$ holes into the model. Since each hole corresponds to one fewer spin degree of freedom and the topological degrees of freedom are neglected, the total number of $\mathbb{Z}_{2}$ degrees of freedom (modes) is $2 N-n-2$. We restrict our attention to the thermodynamic limit of $N_{X, Y} \rightarrow \infty$ and assume that the holes in the model are isolated such that the smallest distance between any two holes is $R \gg 1$.

In the presence of $n>0$ holes, we distinguish two types of plaquettes: hole plaquettes that contain one hole site each and bulk plaquettes that contain no hole sites. Each hole site $l \in \Delta$ is contained by three plaquettes $P_{l}^{x, y, z}$ whose corresponding plaquette operators $W_{P_{l}^{x, y, z}}$ act on the hole site with $\sigma_{l}^{x, y, z}$, respectively. The number of hole plaquettes is therefore $3 n$ and the number of bulk plaquettes is $N-3 n$. From a perturbation theory in $J \ll 1$, there is a finite excitation energy $E_{P} \sim+J^{4}$ for bulk plaquettes and no excitation energy for hole plaquettes. However, at each hole site $l \in \Delta$, there is a finite excitation energy $E_{Q} \sim-J^{8}$ for the hole loop

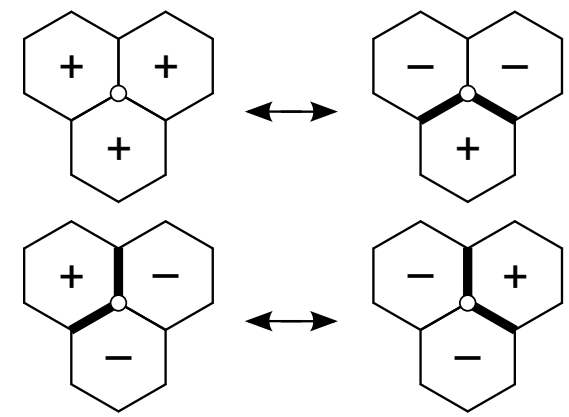

FIG. 3: Simultaneous gauge transformations $D_{l}$ and $\sigma_{l}^{z}$ relating the bond fermion sectors around a hole site $l \in \Delta$ (white dot) when there is no flux bound to the hole. Each bond fermion sector is labeled with the excited bond fermions (thick lines) and the corresponding plaquette operator eigenvalues $( \pm 1)$. Our convention is to consider only the two bond fermion sectors on the left.

$Q_{l}$ surrounding all three hole plaquettes. The negative excitation energy $E_{Q}<0$ means that the hole loop operators $W_{Q_{l}}$ preferentially take eigenvalues -1 in the ground state $\stackrel{14}{\stackrel{14}{ } \text { More }}$ precisely, since the global constraint $\prod_{P} W_{P}=1$ translates into $\prod_{l \in \Delta} W_{Q_{l}}=1$ when no bulk plaquettes are excited, the hole loop operator $W_{Q_{l}}$ is -1 for all hole sites when $n$ is even and for all but one hole sites when $n$ is odd.

Since the hole loop operator is $W_{Q_{l}}=W_{P_{l}^{x}} W_{P_{l}^{y}} W_{P_{l}^{z}}$ in terms of the individual hole plaquette operators, we say that the hole at site $l$ has a flux bound to it if its hole loop operator $W_{Q_{l}}$ takes an eigenvalue -1 rather than +1 . This relation also suggests that each hole has a hole flux mode $Q_{l}$ with a finite excitation energy and two independent hole plaquette modes $P_{l}^{x, z}$ with zero excitation energies. In fact, there is one fewer hole plaquette mode due to the presence of the unphysical hole spin: the four plaquette sectors corresponding to $W_{P_{l}^{x, z}}= \pm 1$ in the hole spin picture are pairwise related by the gauge transformation $\sigma_{l}^{z}$, and the corresponding bond fermion sectors in the Majorana fermion picture are pairwise related by the gauge transformations $D_{l}$ and $\sigma_{l}^{z}$. These gauge transformations are illustrated in Fig. 3. In the following, we use the convention in which the two remaining bond fermion sectors are related to each other by the operator $i b_{l}^{x} b_{l}^{z}$ flipping the $x$ and the $z$ bond fermions around the hole site $l$. When there is no flux bound to the hole, this means that the two remaining plaquette sectors with $W_{P_{l}^{y}}=+1$ are distinguished by $W_{P_{l}^{x}}=W_{P_{l}^{z}}= \pm 1$. In conclusion, if the model contains $n>0$ holes, there are $N-3 n$ bulk plaquette (flux) modes with excitation energies $E_{P} \sim J^{4}$, there are $n-1$ hole flux modes with excitation energies $E_{Q} \sim J^{8}$, and there are $n$ hole plaquette modes with zero excitation energies. Note that the number of independent hole flux modes is reduced by 1 due to the global constraint $\prod_{P} W_{P}=1$.

In the presence of $n>0$ holes, we distinguish two types of fermions: hole fermions and bulk fermions. When $J=0$, hole fermions are localized at dimers that contain one hole site each, while bulk fermions are localized at dimers that contain no hole sites. Since the bulk dimers have Ising interactions $-\sigma_{l}^{z} \sigma_{z(l)}^{z}$, there is a finite excitation energy $E_{f}=2$ for the 
bulk fermions. However, since the Ising interactions of the hole dimers are switched off, there is no excitation energy for the hole fermions. When $J>0$, the bulk fermions delocalize across the entire lattice (see Sec. IVA), but the hole fermions remain localized at their holes. More precisely, each hole fermion wave function forms a wedge of opening angle $\pi / 3$ around its hole and its amplitude decays exponentially with distance. ${ }^{14}$ Since there is one hole fermion for each hole, there are $N-n$ bulk fermion modes with excitation energies $E_{f} \sim 1$, and there are $n-1$ hole fermion modes with zero excitation energies. Note that the number of independent hole fermion modes is reduced by 1 due to the global constraint that $N_{\chi}+N_{f}$ must be even.

The independent $\mathbb{Z}_{2}$ modes of the model with $n>0$ holes are summarized in Table III We distinguish two classes of modes depending on their excitation energies and the scaling of their numbers with $N$ and $n$. The bulk fluxes and the bulk fermions are external (bulk) modes: they have large excitation energies $E \gtrsim J^{4}$ and their numbers scale with the system size $N$. These modes are extremely hard to treat in the thermodynamic limit. Conversely, the hole fluxes, the hole fermions, and the hole plaquettes are internal modes: they have small excitation energies $E \lesssim J^{8}$ and their numbers scale with the hole number $n$. Since these modes are associated with individual holes, it is straightforward to treat them in the limit when the holes are isolated. Due to the different energy scales of the two classes of modes, we can self-consistently neglect the excitations in the high-energy bulk modes, and concentrate only on the low-energy internal modes.

\begin{tabular}{c|c|c|c|c}
\hline \hline Mode type & $\begin{array}{c}\text { Excitation } \\
\text { energy }\end{array}$ & $\begin{array}{c}\text { Number } \\
\text { of modes }\end{array}$ & $\begin{array}{c}\text { Quantum } \\
\text { number }\end{array}$ & $\begin{array}{c}\text { Global } \\
\text { constraint }\end{array}$ \\
\hline Bulk fermion & $\sim 1$ & $N-n$ & & \\
Bulk flux & $\sim J^{4}$ & $N-3 n$ & & \\
Hole flux & $\sim J^{8}$ & $n-1$ & $h=\{0,1\}$ & $\sum_{j} h_{j}=$ even \\
Hole fermion & 0 & $n-1$ & $q=\{0,1\}$ & $\sum_{j} q_{j}=$ even \\
Hole plaquette & 0 & $n$ & $p=\{0,1\}$ & \\
\hline \hline
\end{tabular}

TABLE III: Energy hierarchy of independent $\mathbb{Z}_{2}$ modes in the model with $n>0$ holes. For the internal modes, the corresponding quantum numbers are also specified along with any global constraints on them. The total number of modes is $2 N-n-2$ as expected.

Each hole in the model has three internal modes, and we characterize these three internal modes with three $\mathbb{Z}_{2}$ quantum numbers $h, q$, and $p$. The flux quantum number is $h=1$ if the hole has a flux bound to it and $h=0$ otherwise. The fermion quantum number is $q=1$ if the corresponding hole fermion is excited and $q=0$ otherwise. The meaning of the plaquette quantum number $p$ depends on the flux quantum number: if $h=0$, then $p=0$ means no hole plaquette excitations and $p=1$ means two hole plaquette excitations in two neighboring stripes, while if $h=1$, then $p=0$ means one hole plaquette excitation in an even stripe and $p=1$ means one hole plaquette excitation in an odd stripe. The corresponding bond fermion sectors are shown in Fig. 4. Importantly, the distinction between even and odd stripes ensures that $N_{\chi}$ is always
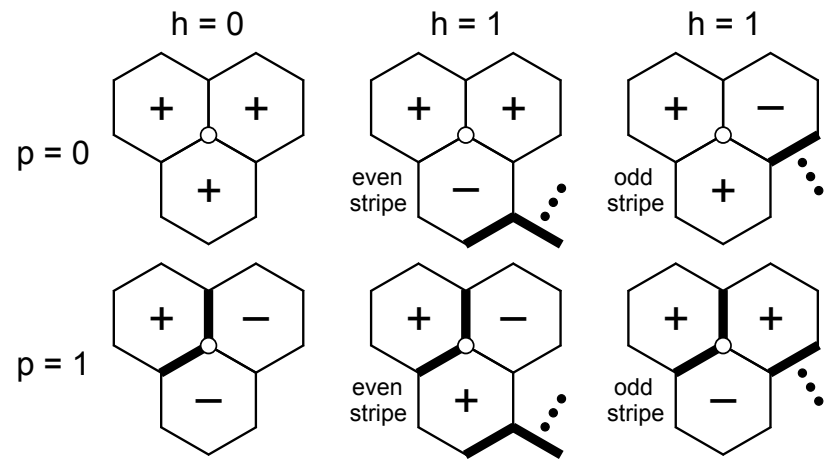

FIG. 4: Bond fermion sectors around a hole site $l \in \Delta$ (white dot) for different combinations of the flux quantum number $h=\{0,1\}$ and the plaquette quantum number $p=\{0,1\}$. Each bond fermion sector is labeled with the excited bond fermions (thick lines) and the corresponding plaquette operator eigenvalues $( \pm 1)$. For $h=1$, there are two cases depending on whether $P_{l}^{z}$ is in an even stripe or in an odd stripe. The triple dots indicate a string of excited bond fermions connecting two holes with $h=1$.

even. In the case of $n$ holes labeled $j=\{1,2, \ldots, n\}$, there are $3 n$ internal modes characterized by $3 n$ quantum numbers $h_{j}, q_{j}$, and $p_{j}$. Since fluxes and fermions can only be excited pairwise, the quantum numbers $h_{j}$ and $q_{j}$ are not fully independent from each other. In particular, the global constraint $\prod_{P} W_{P}=1$ translates into $\sum_{j} h_{j}=$ even, while the global constraint that $N_{\chi}+N_{f}$ is even, or equivalently, that $N_{f}$ is even translates into $\sum_{j} q_{j}=$ even. The various formulations of these two global constraints are presented in Table IV

\begin{tabular}{c|c|c}
\hline \hline & Flux constraint & Fermion constraint \\
\hline Physical spins & $\prod_{P} W_{P}=1$ & $\prod_{l \in A} \lambda_{l} \prod_{P \in \mu} W_{P}=1$ \\
Majorana fermions & Automatically satisfied & $N_{\chi}+N_{f}=$ even \\
E / M charges & $N_{e}+N_{m}=$ even & $N_{m}=$ even \\
Quantum numbers & $\sum_{j} h_{j}=$ even & $\sum_{j} q_{j}=$ even \\
\hline \hline
\end{tabular}

TABLE IV: Formulations of the two essential global constraints in terms of the physical spins, the Majorana fermions, the electric / magnetic charges, and the internal quantum numbers.

We are now ready to write down the ground states $\left|\Omega_{h, q, p}^{\Delta}\right\rangle$ that correspond to the different values of the internal quantum quantum numbers. Using the method of Sec. IV A each ground state is obtained from the vacuum state $|0\rangle$ by a projection onto an appropriate subspace. Formally, the physical ground state for $n>0$ holes at sites $\Delta=\left\{l_{j}\right\}$ with quantum numbers $h \equiv\left\{h_{j}\right\}, q \equiv\left\{q_{j}\right\}$, and $p \equiv\left\{p_{j}\right\}$ reads as

$$
\left|\Omega_{h, q, p}^{\Delta}\right\rangle=\mathcal{Q}_{\Delta} \mathcal{P} \mathcal{F}_{q ; h} \mathcal{B}_{p} \mathcal{X}_{h}|0\rangle \text {. }
$$

Before enforcing the gauge constraints with the projection operators $\mathcal{P}$ and $\mathcal{Q}_{\Delta}$, the vacuum state $|0\rangle$ is acted upon by several operators setting the bond fermion and the matter fermion sectors. The first operator $\mathcal{X}_{h}$ is responsible for binding fluxes to all holes with $h_{j}=1$. Mathematically, $\mathcal{X}_{h}$ is an appropriate product of $\left(\chi_{l}^{\alpha}\right)^{\dagger}$ operators along a set of strings connecting the holes with $h_{j}=1$ pairwise. Note that the global 
constraint $\sum_{j} h_{j}=$ even ensures that the holes with $h_{j}=1$ can always be paired up. Importantly, we choose $\mathcal{X}_{h}$ such that it does not excite any $z$ bond fermions and creates the excited plaquette in an even stripe for each hole (see Fig. 4). In this case, $\mathcal{X}_{h}$ is a product of an even number of $\left(\chi_{l}^{\alpha}\right)^{\dagger}$ operators, and therefore it excites an even number of bond fermions. The remaining two operators in Eq. (20) are given by

$$
\begin{aligned}
\mathcal{B}_{p} & =\prod_{j=1}^{n}\left(i b_{l_{j}}^{x} b_{l_{j}}^{z}\right)^{p_{j}} \\
\mathcal{F}_{q ; h} & =\prod_{k=n+1}^{N}\left(\phi_{k} \phi_{k}^{\dagger}\right) \prod_{j=1}^{n}\left(\phi_{j}^{1-q_{j}} \phi_{j}^{\dagger} \phi_{j}^{q_{j}}\right) \prod_{j=1}^{n}\left[f_{\tilde{z}\left(l_{j}\right)}^{\dagger}\right]^{q_{j}},
\end{aligned}
$$

where $\tilde{z}(l)=l$ if $l \in A$ and $\tilde{z}(l)=z(l)$ if $l \in B$. The operator $\mathcal{B}_{p}$ sets the bond fermion sector by flipping an even number of bond fermions around the hole sites, while the operator $\mathcal{F}_{q ; h}$ projects onto one of the ground states in the given bond fermion sector. The original matter fermions $f_{\tilde{z}\left(l_{j}\right)}^{\dagger}$ are required only to ensure that $\mathcal{F}_{q ; h}$ does not project to zero in the isolated dimer limit. The free matter fermions $\phi_{k}$ are obtained from the matrix $M$ in Eq. [8]: there are $n$ hole fermions $\phi_{k}$ with $1 \leq k \leq n$ that have zero energies and $N-n$ bulk fermions $\phi_{k}$ with $n+1 \leq k \leq N$ that have finite energies $E_{f} \sim 1$. We label the hole fermions consistently such that the hole fermion $\phi_{j}$ is localized around the hole site $l_{j}$. Note that the matrix $M$ is in general a function of the bond fermions excited by $\mathcal{X}_{h}$, and therefore $\mathcal{F}_{q ; h}$ depends on the flux quantum numbers $h_{j}$ via the free matter fermions $\phi_{k}$. On the other hand, the bond fermions flipped by $\mathcal{B}_{p}$ correspond to bonds with switched-off interactions, and therefore $\mathcal{F}_{q ; h}$ does not depend on the plaquette quantum numbers $p_{j}$.

\begin{tabular}{c|c|c}
\hline \hline \multicolumn{2}{c|}{ Hole type } & Superselection sector \\
\hline \multirow{2}{*}{$h=0$} & $q=0$ & Trivial $(1)$ \\
& $q=1$ & Combined $(e \times m)$ \\
\hline \multirow{2}{*}{$h=1$} & $q=0$ & Electric $(e)$ \\
& $q=1$ & Magnetic $(m)$ \\
\hline \hline
\end{tabular}

TABLE V: Superselection sectors of holes with flux quantum numbers $h=\{0,1\}$ and fermion quantum numbers $q=\{0,1\}$.

It is useful to interpret the internal quantum numbers in the isolated dimer limit. In this limit, the free matter fermions $\phi_{k}$ are identical to the original matter fermions $f_{l}$, and therefore the second operator in Eq. (21) takes the simplified form $\mathcal{F}_{q} \equiv \mathcal{F}_{q ; h}=\prod_{j=1}^{n}\left[f_{\tilde{z}\left(l_{j}\right)}^{\dagger}\right]^{q_{j}}$. Note that the matrix $M$ is no longer a function of the $x$ and $y$ bond fermions excited by $\mathcal{X}_{h}$, and therefore $\mathcal{F}_{q}$ becomes independent of the flux quantum numbers $h_{j}$. For a single isolated hole at site $l$ with quantum numbers $h, q$, and $p$, the hole dimer operator is then $\lambda_{l}=\sigma_{l}^{z} \sigma_{z(l)}^{z}=(-1)^{q+p}$. Since the product of the hole plaquette operators is $\prod_{P_{l} \in \eta} W_{P}=(-1)^{h+p}$ in even stripes and $\prod_{P_{l} \in \mu} W_{P}=(-1)^{p}$ in odd stripes, we conclude that the different combinations of the quantum numbers $h$ and $q$ are in one-to-one correspondence with the different superse- lection sectors that the hole can belong to. This correspondence is presented in Table $\mathrm{V}$ Note that if the bulk modes are not excited, isolated holes can indeed be thought of as isolated excitation clusters with well-defined superselection sectors. Furthermore, since the projection operator $\mathcal{Q}_{\Delta}$ enforces $\sigma_{l}^{z}=+1$ at the hole site $l$, there is a finite local magnetization $\sigma_{z(l)}^{z}=(-1)^{q+p}$ at the neighboring site $z(l) !^{14}$ This magnetization can be reversed by applying the transformation $\sigma^{x, z} \rightarrow-\sigma^{x, z}$ to all spins except the hole spin. On the other hand, such a discrete spin rotation is also a symmetry of the model: it flips the hole plaquettes $P_{l}^{x, z}$ and changes the sign of the hole dimer operator $\lambda_{l}$. It therefore corresponds to a switch in the plaquette quantum number $p$ only. To summarize, the flux and the fermion quantum numbers determine the superselection sector, while the plaquette quantum number determines the local magnetization around the hole. Importantly, these results are also valid in the case of $J>0$ when $(-1)^{q+p}$ is equal to the product of dimer operators taken over a sufficiently large region around the hole site $l$.

\section{Interactions and bound states}

We now discuss the interactions between two holes at a finite distance $R$ away from each other. In general, the groundstate energy is given by $\Gamma_{0}=-\sum_{k} S_{k}$, where the $N$ singular values $S_{k}$ are obtained from the matrix $M$ in Eq. (8). In the limit of $R \rightarrow \infty$, there are two vanishing singular values $S_{1}=S_{2}=0$ corresponding to the two hole fermions, and the ground-state energy $\Gamma_{0}(\infty)$ is determined by the sum of the remaining $N-2$ non-vanishing singular values. When $R$ is finite, the interaction energy between the two holes is defined as the change in the ground-state energy with respect to that in the $R \rightarrow \infty$ limit: $\Delta \Gamma_{0} \equiv \Gamma_{0}(R)-\Gamma_{0}(\infty)$.

The interaction energy $\Delta \Gamma_{0}$ has two contributions arising from two distinct interaction mechanisms. First, the sum of the $N-2$ non-vanishing singular values is changed by perturbative terms similar to those in Eq. 15. Second, the singular value $S_{2}$ also becomes non-vanishing due to a hybridization between the two hole fermions. ${ }^{14}$ The first contribution $\Delta \Gamma_{0}^{(1)}$ is non-zero for both sublattices and all directions, while the second contribution $\Delta \Gamma_{0}^{(2)}$ is non-zero only if the two holes are in opposite sublattices and their relative direction lies in the wedge of opening angle $\pi / 3$ such that each hole fermion wave function has a finite amplitude at the hole site of the other hole (see Fig. 57. Importantly, the wedges for the two holes in the opposite sublattices point in opposite directions, and therefore this condition for the relative direction is identical from the point of view of both holes.

Since the two contributions decay as $\Delta \Gamma_{0}^{(1)} \sim J^{2 R}$ and $\Delta \Gamma_{0}^{(2)} \sim J^{R}$ with the distance $R$, the second contribution is the dominant one at large distances. From the lowest-order perturbation theory in $J \ll 1$ around the isolated dimer limit, this contribution takes the general form

$$
\Delta \Gamma_{0}^{(2)}=-\frac{R !}{R_{x} ! R_{y} !} J^{R}
$$


(a)

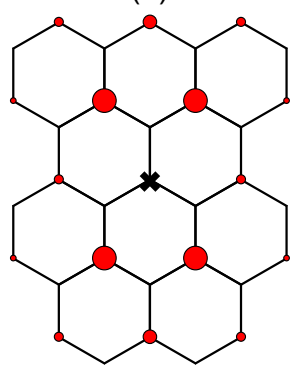

(b)

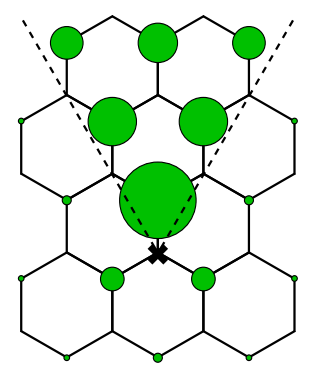

FIG. 5: (Color online) Absolute interaction energy $\left|\Delta \Gamma_{0}\right|$ between two holes as a function of their relative position when $J_{z}=1.0$ and $J \equiv J_{x}=J_{y}=0.2$. One hole is fixed (black cross) and the other one is moved around (red and green dots). Each dot has an area proportional to $\sqrt{\left|\Delta \Gamma_{0}\right|}$. The interaction is either repulsive with $\Delta \Gamma_{0}>0$ (a) or attractive with $\Delta \Gamma_{0}<0$ (b). The wedge of opening angle $\pi / 3$ is marked by a dashed line.

where the string of shortest length $R \equiv R_{x}+R_{y}$ connecting the two holes contains $R_{x}$ bonds of $x$ type and $R_{y}$ bonds of $y$ type 14 The first contribution can also be calculated from a perturbation theory in $J \ll 1$, but its general form is more complicated. In particular, $\Delta \Gamma_{0}^{(1)}$ can take both signs: the largest negative result $\Delta \Gamma_{0}^{(1)}=-J^{2} / 4$ is found when the two holes are at nearest-neighbor sites connected by an $x$ or a $y$ bond, while the largest positive result $\Delta \Gamma_{0}^{(1)}=J^{2} / 4$ is found when the two holes are at next-nearest-neighbor sites connected by a $z$ bond and an $x$ or a $y$ bond. The interaction energy $\Delta \Gamma_{0}=\Delta \Gamma_{0}^{(1)}+\Delta \Gamma_{0}^{(2)}$ is always positive when the two holes are in the same sublattice and always negative when the two holes are in opposite sublattices. The absolute values of the interaction energies are plotted in Fig. 5

Importantly, the first interaction mechanism corresponding to $\Delta \Gamma_{0}^{(1)}$ is diagonal in the quantum numbers $h, q$, and $p$, while the second interaction mechanism corresponding to $\Delta \Gamma_{0}^{(2)}$ is diagonal only in $h$ and $p$ but not in $q$. In particular, if we set $h_{1,2}=p_{1,2}=0$ for simplicity and label the remaining four ground states $\left|\Omega_{h, q, p}^{\Delta}\right\rangle$ with the fermion quantum numbers as $\left|q_{1}, q_{2}\right\rangle \equiv\left|\Omega_{q_{1}, q_{2}}\right\rangle$, the second interaction has identical matrix elements $\sim J^{R}$ between the states $|0,0\rangle$ and $|1,1\rangle$, and between the states $|0,1\rangle$ and $|1,0\rangle$. This implies that the eigenstates are in fact $(|0,0\rangle \pm|1,1\rangle) / \sqrt{2}$ and $(|0,1\rangle \pm|1,0\rangle) / \sqrt{2}$. In the strict sense, the fermion quantum numbers $q$ are then no longer valid quantum numbers in the presence of hole interactions. However, since the interaction is exponentially small when the holes are far apart, they are still practically valid quantum numbers as they are conserved within an exponentially large timescale $\sim J^{-R}$.

Since the attractive interaction between holes in opposite sublattices is stronger than the repulsive interaction between holes in the same sublattice, the overall interaction between two holes is attractive. The most negative interaction energy $\Delta \Gamma_{0}=-1$ is found when the two holes are at nearestneighbor sites connected by a $z$ bond. In the absence of other interactions, this attraction leads to pair formation, where the holes in the model form bound pairs along $z$ bonds. It is then useful to investigate how these hole pairs interact with each other. The interaction energy $\Delta \Gamma_{0}^{\prime} \equiv \Gamma_{0}^{\prime}(R)-\Gamma_{0}^{\prime}(\infty)$ between two hole pairs is completely analogous to that between two single holes. In this case, there are two vanishing singular values for all distances $R$, and the only contribution to the interaction energy comes from the change in the remaining $N-2$ non-vanishing singular values. From a perturbation theory around the isolated dimer limit, we obtain that the interaction energy between two hole pairs is always negative and decays as $\Delta \Gamma_{0}^{\prime} \sim J^{2 R}$ with the distance $R$. The most negative interaction energy $\Delta \Gamma_{0}^{\prime}=-J^{2} / 4$ is found when two holes from the respective hole pairs are at nearest-neighbor sites connected by an $x$ or a $y$ bond.

In the absence of other interactions, the attraction between hole pairs leads to phase separation, where the holes are all bound together to form a large cluster. However, both single holes and hole pairs are positively charged, and therefore they are also subject to a Coulomb repulsion. Since the attraction between single holes is stronger than that between hole pairs, we can distinguish three complementary regimes in the behavior of the model. If the Coulomb repulsion is weaker than the attraction between hole pairs, the model phase separates. If the Coulomb repulsion is stronger than the attraction between hole pairs but weaker than that between single holes, the elementary particles of the model are hole pairs. If the Coulomb repulsion is stronger than the attraction between single holes, the elementary particles of the model are single holes. In the following, we restrict our attention to single holes and implicitly assume a sufficiently strong Coulomb repulsion such that the model is in the appropriate regime.

\section{Robustness against local perturbations}

It is useful to discuss the applicability of the internal quantum numbers when a local perturbation is applied to the model with $n>0$ holes. We first notice that two arguments are apparently in conflict with each other. On one hand, the quantum numbers $q$ and $h$ are expected to be robust against local perturbations as they are related to the superselection sectors of the model (see Secs. IVB and VB. On the other hand, the quantum numbers $q$ are not strictly conserved in the presence of hole interactions (see Sec. VC). Note that the dimensionless coupling strength $J \ll 1$ is a local perturbation in the language of the isolated dimer limit.

The resolution of this apparent conflict is that local perturbations assemble into non-local strings at higher orders of perturbation theory. If two holes (excitation clusters) are connected by such a string, only the combined superselection sector is conserved, while the individual superselection sectors can change. However, when the two holes are at a distance $R$ away from each other, such a string can be assembled only at $R$-th order of perturbation theory. For a local perturbation of strength $\delta E$ that creates excitations with energies $E_{0}$, the perturbative term responsible for changing the superselection sector is then $\sim\left(\delta E / E_{0}\right)^{R}$. This means that the superselection sector is conserved within a timescale $\sim\left(E_{0} / \delta E\right)^{R}$ that 
(a)<smiles>C1CC2CCC3CCC4CCC5CCC(C1)C45C23</smiles><smiles>[194In]</smiles>

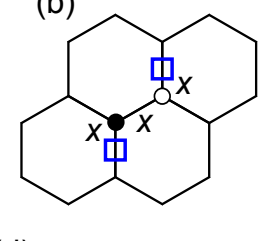

(e)
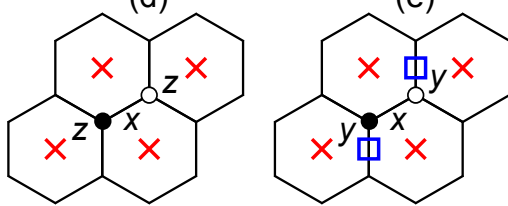

FIG. 6: (Color online) Effects of the Heisenberg terms $\sigma_{l}^{z} \sigma_{l^{\prime}=z(l)}^{z}$ (a), $\sigma_{l}^{x} \sigma_{l^{\prime}=x(l)}^{x}$ (b), $\sigma_{l}^{x} \sigma_{l^{\prime}=z(l)}^{x}(\mathrm{c}), \sigma_{l}^{z} \sigma_{l^{\prime}=x(l)}^{z}$ (d), and $\sigma_{l}^{y} \sigma_{l^{\prime}=x(l)}^{y}$ (e) on the plaquettes and the dimers (fermions) around two neighboring sites $l$ (white dot) and $l^{\prime}$ (black dot). Flipped plaquettes are marked by red crosses and flipped dimers are marked by blue rectangles.

is exponentially large when $\delta E \ll E_{0}$ and $R \gg 1$. Note that the interaction term $\sim J^{R}$ in Sec. $\mathrm{VC}$ is recovered as a special case with $\delta E \sim J$ and $E_{0} \sim 1$. Since local perturbations excite bulk fluxes with energies $E_{P} \sim J^{4}$ and bulk fermions with energies $E_{f} \sim 1$ in general, we conclude that the quantum numbers $h$ and $q$ are robust against arbitrary local perturbations of strength $\delta E \ll J^{4}$ as long as the holes are sufficiently far away from each other.

It is instructive to examine an explicit example for the conservation of the internal quantum numbers in the presence of a local perturbation. To this end, we perturb the Kitaev honeycomb model with Heisenberg interactions. The contribution of this perturbation to the Hamiltonian reads as

$$
\delta H=\delta E \sum_{\left\langle l, l^{\prime}\right\rangle}\left(\sigma_{l}^{x} \sigma_{l^{\prime}}^{x}+\sigma_{l}^{y} \sigma_{l^{\prime}}^{y}+\sigma_{l}^{z} \sigma_{l^{\prime}}^{z}\right),
$$

where $\left\langle l, l^{\prime}\right\rangle$ indicates a summation over bonds, or equivalently, over pairs of neighboring sites. Based on the type of the bond and the spin components coupled, there are nine types of terms in $\delta H$, and these types can be divided into four distinct classes in the isolated dimer limit. The terms $\sigma_{l}^{z} \sigma_{z(l)}^{z}$ only renormalize the coupling strength $J_{z}$ on the $z$ bonds, and therefore do not flip any plaquettes or dimers (fermions). The terms $\sigma_{l}^{x} \sigma_{x(l)}^{x}$ and $\sigma_{l}^{y} \sigma_{y(l)}^{y}$ correspond to the usual couplings with strengths $J_{x, y}$ on the $x$ and $y$ bonds, and therefore flip no plaquettes but two dimers each. The terms $\sigma_{l}^{x} \sigma_{z(l)}^{x}, \sigma_{l}^{y} \sigma_{z(l)}^{y}$, $\sigma_{l}^{z} \sigma_{x(l)}^{z}$, and $\sigma_{l}^{z} \sigma_{y(l)}^{z}$ flip no dimers and four plaquettes each, while the terms $\sigma_{l}^{y} \sigma_{x(l)}^{y}$ and $\sigma_{l}^{x} \sigma_{y(l)}^{x}$ flip two dimers and four plaquettes each. The effects of these types of terms are illustrated in Fig. 6. Since the perturbative terms flip either zero or two dimers, the number of broken dimers has a conserved parity, and therefore the parity of $q+p$ does not change either (see Sec. VB. Since they either flip zero plaquettes or they flip two plaquettes in even stripes and two plaquettes in odd stripes, the numbers of excited plaquettes in even and in odd stripes both have conserved parities, and therefore the parities of $h+p$ and $p$ do not change either. We conclude that the quantum numbers $h, q$, and $p$ are all conserved in the presence of a Heisenberg perturbation if its strength satisfies $\delta E \ll J^{4}$ and the holes are sufficiently far apart.

\section{ISOLATED MOBILE HOLES}

\section{A. Hopping formalism}

We consider a hole hopping model in which the holes introduced into the Kitaev honeycomb model can propagate via nearest-neighbor hopping. Formally, a spin one-half particle at a site $l^{\prime}$ neighboring an empty hole site $l$ can hop from $l^{\prime}$ to $l$ with an amplitude $-t$. We assume that the spin state of the particle is not affected by the hopping. In the hole spin picture, the hopping then exchanges the hole spin at $l$ with the actual spin at $l^{\prime}$, and this process can be represented by an exchange operator that takes the form

$$
\begin{aligned}
\mathcal{E}_{l, l^{\prime}} & =\frac{1}{2}\left(1+\sigma_{l}^{x} \sigma_{l^{\prime}}^{x}+\sigma_{l}^{y} \sigma_{l^{\prime}}^{y}+\sigma_{l}^{z} \sigma_{l^{\prime}}^{z}\right) \\
& =\frac{1}{2}\left(1+b_{l}^{y} b_{l^{\prime}}^{y} b_{l}^{z} b_{l^{\prime}}^{z}+b_{l}^{z} b_{l^{\prime}}^{z} b_{l}^{x} b_{l^{\prime}}^{x}+b_{l}^{x} b_{l^{\prime}}^{x} b_{l}^{y} b_{l^{\prime}}^{y}\right) .
\end{aligned}
$$

In the following, we restrict our attention to the regime of slow hopping, where the hopping amplitude is much smaller than the excitation energies of the bulk modes. We can then neglect the excitations in the bulk modes and consider only the ground states $\left|\Omega_{h, q, p}^{\Delta}\right\rangle$ corresponding to the internal modes. Since the bulk modes are bulk fluxes with energies $E_{P} \sim J^{4}$ and bulk fermions with energies $E_{f} \sim 1$ in general, the condition of slow hopping becomes $t \ll J^{4}$.

For simplicity, we restrict our attention to only $n=2$ isolated holes at sites $l_{1,2}$. However, more holes are assumed to be present in the background so that the quantum numbers $h_{1,2}$ and $q_{1,2}$ can be chosen independently without violating the global constraints. We consider the hopping process in which the hole at site $l_{1}$ hops to a neighboring site $l_{1}^{\prime}$. The set of hole sites is $\Delta=\left\{l_{1}, l_{2}\right\}$ before the hopping and $\Delta^{\prime}=\left\{l_{1}^{\prime}, l_{2}^{\prime} \equiv l_{2}\right\}$ after the hopping. The ground states corresponding to the hole positions $\Delta$ and $\Delta^{\prime}$ take the forms

$$
\begin{aligned}
\left|\Omega_{h, q, p}\right\rangle & \equiv\left|\Omega_{h, q, p}^{\Delta}\right\rangle=\mathcal{Q}_{\Delta} \mathcal{P} \mathcal{F}_{q ; h} \mathcal{B}_{p} \mathcal{X}_{h}|0\rangle, \\
\left|\bar{\Omega}_{h^{\prime}, q^{\prime}, p^{\prime}}\right\rangle & \equiv\left|\Omega_{h^{\prime}, q^{\prime}, p^{\prime}}^{\Delta}\right\rangle=\mathcal{Q}_{\Delta^{\prime}} \mathcal{P} \overline{\mathcal{F}}_{q^{\prime} ; h^{\prime}} \overline{\mathcal{B}}_{p^{\prime}} \overline{\mathcal{X}}_{h^{\prime}}|0\rangle,
\end{aligned}
$$

where the operators $\overline{\mathcal{X}}_{h^{\prime}}, \overline{\mathcal{B}}_{p^{\prime}}$, and $\overline{\mathcal{F}}_{q^{\prime} ; h^{\prime}}$ are completely analogous to $\mathcal{X}_{h}, \mathcal{B}_{p}$, and $\mathcal{F}_{q ; h}$ as defined in Sec. $\mathrm{VB}$. Since different bonds have switched-off interactions before and after the hopping, the operators $\mathcal{F}_{q ; h}$ and $\overline{\mathcal{F}}_{q^{\prime} ; h^{\prime}}$ contain different free matter fermions $\phi_{k}$ and $\phi_{k}$. By considering the hopping between the respective ground states $\left|\Omega_{h, q, p}\right\rangle$ and $\left|\bar{\Omega}_{h^{\prime}, q^{\prime}, p^{\prime}}\right\rangle$, the effective hopping amplitude becomes a finite-dimensional matrix. The elements of this matrix are given by

$$
T_{h, q, p}^{h^{\prime}, q^{\prime}, p^{\prime}}=-\frac{t\left\langle\bar{\Omega}_{h^{\prime}, q^{\prime}, p^{\prime}}\left|\mathcal{E}_{l_{1}, l_{1}^{\prime}}\right| \Omega_{h, q, p}\right\rangle}{\sqrt{\left\langle\bar{\Omega}_{h^{\prime}, q^{\prime}, p^{\prime}} \mid \bar{\Omega}_{h^{\prime}, q^{\prime}, p^{\prime}}\right\rangle\left\langle\Omega_{h, q, p} \mid \Omega_{h, q, p}\right\rangle}},
$$

where the ground-state norms in the denominator are required because the ground states $\left|\Omega_{h, q, p}\right\rangle$ and $\left|\bar{\Omega}_{h^{\prime}, q^{\prime}, p^{\prime}}\right\rangle$ are not properly normalized in general. 


\section{B. General hopping properties}

We notice that the non-trivial terms in the exchange operator $\mathcal{E}_{l_{1}, l_{1}^{\prime}}$ are the Heisenberg terms in Eq. 23). It is therefore directly implied by the results in Sec. VD that the quantum numbers $h, q$, and $p$ are all conserved by the hopping. Note that the exchange operator $\mathcal{E}_{l_{1}, l_{1}^{\prime}}$ can in principle change the plaquette (matter fermion) sector in at most two inequivalent ways (see Fig. 6). The plaquette (matter fermion) sector after the hopping is then uniquely determined by that before the hopping via the ground-state constraint that no excited plaquettes (matter fermions) are allowed to be left behind. Mathematically, the conservation of the quantum numbers means that the effective hopping matrix is diagonal.

Furthermore, the diagonal hopping matrix elements that differ only in their plaquette quantum numbers $p$ are all identical to each other. Physically, this property follows from the discrete spin-rotation symmetry discussed in Sec. VB and the fact that the corresponding transformation switches $p$. However, it can also be shown explicitly by noticing that $\mathcal{B}_{p}^{\dagger} \mathcal{B}_{p}=1$ and $\overline{\mathcal{B}}_{p}^{\dagger} \mathcal{E}_{l_{1}, l_{1}^{\prime}} \mathcal{B}_{p}=\mathcal{E}_{l_{1}, l_{1}^{\prime}}$ for all $p$. We therefore conclude that the effective hopping matrix elements are independent of the plaquette quantum numbers $p$ and take the general form

$$
\begin{gathered}
T_{h, q, p}^{h^{\prime}, q^{\prime}, p^{\prime}}=\delta_{h^{\prime}, h} \delta_{q^{\prime}, q} \delta_{p^{\prime}, p} T_{h, q}, \\
T_{h, q}=-\frac{t\left\langle\bar{\Omega}_{h, q}\left|\mathcal{E}_{l_{1}, l_{1}^{\prime}}\right| \Omega_{h, q}\right\rangle}{\sqrt{\left\langle\bar{\Omega}_{h, q} \mid \bar{\Omega}_{h, q}\right\rangle\left\langle\Omega_{h, q} \mid \Omega_{h, q}\right\rangle}},
\end{gathered}
$$

where $\left|\Omega_{h, q}\right\rangle \equiv\left|\Omega_{h, q, p=0}\right\rangle$ and $\left|\bar{\Omega}_{h, q}\right\rangle \equiv\left|\bar{\Omega}_{h, q, p=0}\right\rangle$. In the following, we simplify our calculations by considering only these ground states with $p_{1}=p_{2}=0$.

Now we derive a formula for the effective hopping matrix element $\tilde{T}_{h, q}$ in the important case when the bond fermion sector (plaquette sector) is conserved by the hopping. This condition is equivalent to $\mathcal{X}_{h}=\overline{\mathcal{X}}_{h}$, and it is always satisfied in the case of trivial flux quantum numbers $h_{1}=h_{2}=0$ when $\mathcal{X}_{h=0}=\overline{\mathcal{X}}_{h=0}=1$. Since $\left\langle 0\left|\mathcal{X}_{h}^{\dagger} \mathcal{X}_{h}\right| 0\right\rangle=1$ in general, the ground-state norms in the denominator of Eq. (28) become

$$
\begin{aligned}
\left\langle\Omega_{h, q} \mid \Omega_{h, q}\right\rangle & =\left\langle 0\left|\mathcal{X}_{h}^{\dagger} \mathcal{F}_{q ; h}^{\dagger} \mathcal{P} \mathcal{Q}_{\Delta} \mathcal{F}_{q ; h} \mathcal{X}_{h}\right| 0\right\rangle=\frac{1}{2^{2 N+2}}\left\langle 0\left|\mathcal{F}_{q ; h}^{\dagger} \mathcal{F}_{q ; h}\right| 0\right\rangle \\
\left\langle\bar{\Omega}_{h, q} \mid \bar{\Omega}_{h, q}\right\rangle & =\left\langle 0\left|\mathcal{X}_{h}^{\dagger} \overline{\mathcal{F}}_{q ; h}^{\dagger} \mathcal{P} \mathcal{Q}_{\Delta^{\prime}} \overline{\mathcal{F}}_{q ; h} \mathcal{X}_{h}\right| 0\right\rangle=\frac{1}{2^{2 N+2}}\left\langle 0\left|\overline{\mathcal{F}}_{q ; h}^{\dagger} \overline{\mathcal{F}}_{q ; h}\right| 0\right\rangle
\end{aligned}
$$

By assuming $l_{1} \in A$ without loss of generality, using the property $\sigma_{l_{1}^{\prime}}^{z} \mathcal{E}_{l_{1}, l_{1}^{\prime}} \sigma_{l_{1}}^{z}=\mathcal{E}_{l_{1}, l_{1}^{\prime}}$, and keeping only the terms in $\mathcal{E}_{l_{1}, l_{1}^{\prime}}$ that do not change the plaquette sector when $\alpha \equiv \alpha_{l_{1}, l_{1}^{\prime}}=\{x, y, z\}$, the ground-state overlap in the numerator of Eq. (28) becomes

$$
\begin{aligned}
\left\langle\bar{\Omega}_{h, q}\left|\mathcal{E}_{l_{1}, l_{1}^{\prime}}\right| \Omega_{h, q}\right\rangle & =\left\langle 0\left|\mathcal{X}_{h}^{\dagger} \overline{\mathcal{F}}_{q ; h}^{\dagger} \mathcal{P} \mathcal{Q}_{\Delta^{\prime}} \mathcal{E}_{l_{1}, l_{1}^{\prime}} \mathcal{Q}_{\Delta} \mathcal{F}_{q ; h} \mathcal{X}_{h}\right| 0\right\rangle=\frac{1}{8}\left\langle 0\left|\mathcal{X}_{h}^{\dagger} \overline{\mathcal{F}}_{q ; h}^{\dagger} \mathcal{P}\left(1+b_{l_{1}}^{\alpha} b_{l_{1}^{\prime}}^{\alpha} c_{l_{1}} c_{l_{1}^{\prime}}\right) \mathcal{F}_{q ; h} \mathcal{X}_{h}\right| 0\right\rangle \\
& =\frac{1}{2^{2 N+3}}\left\langle 0\left|\mathcal{X}_{h}^{\dagger} \overline{\mathcal{F}}_{q ; h}^{\dagger}\left(1+b_{l_{1}}^{\alpha} b_{l_{1}^{\prime}}^{\alpha} c_{l_{1}} c_{l_{1}^{\prime}}\right) \mathcal{F}_{q ; h} \mathcal{X}_{h}\right| 0\right\rangle=\frac{1}{2^{2 N+3}}\left\langle 0\left|\overline{\mathcal{F}}_{q ; h}^{\dagger}\left(1-i u_{l_{1}, l_{1}^{\prime}} c_{l_{1}} c_{l_{1}^{\prime}}\right) \mathcal{F}_{q ; h}\right| 0\right\rangle
\end{aligned}
$$

Note that $u_{l_{1}, l_{1}^{\prime}} \equiv\left\langle 0\left|\mathcal{X}_{h}^{\dagger} \hat{u}_{l_{1}, l_{1}^{\prime}} \mathcal{X}_{h}\right| 0\right\rangle$ is determined by the bond fermion sector. Finally, the effective hopping matrix element in the case of a conserved bond fermion sector (plaquette sector) takes the form

$$
\tilde{T}_{h, q}=-\frac{t\left\langle 0\left|\overline{\mathcal{F}}_{q ; h}^{\dagger}\left(1-i u_{l_{1}, l_{1}^{\prime}} c_{l_{1}} c_{l_{1}^{\prime}}\right) \mathcal{F}_{q ; h}\right| 0\right\rangle}{2 \sqrt{\left\langle 0\left|\overline{\mathcal{F}}_{q ; h}^{\dagger} \overline{\mathcal{F}}_{q ; h}\right| 0\right\rangle\left\langle 0\left|\mathcal{F}_{q ; h}^{\dagger} \mathcal{F}_{q ; h}\right| 0\right\rangle}}
$$

Since the operators $\mathcal{F}_{q ; h}^{(\dagger)}$ and $\overline{\mathcal{F}}_{q ; h}^{(\dagger)}$ are all simple products of matter fermion operators, the vacuum expectation values in Eq. 31 can be evaluated using Wick's theorem. The state $|0\rangle$ is the vacuum of the original matter fermions $f_{l}$, and the orthogonal matrices $U$ and $V$ are therefore used to express the free matter fermions $\phi_{k}$ and $\bar{\phi}_{k}$ in terms of $f_{l}$.

\section{Hopping in the isolated dimer limit}

We now consider the isolated dimer limit $(J=0)$ and evaluate the effective hopping matrix elements explicitly. In this limit, the operators $\mathcal{F}_{q} \equiv \mathcal{F}_{q ; h}$ and $\overline{\mathcal{F}}_{q} \equiv \overline{\mathcal{F}}_{q ; h}$ no longer depend on $h$ and take the simplified forms $\mathcal{F}_{q}=\left[f_{\tilde{z}\left(l_{1}\right)}^{\dagger}\right]^{q_{1}}\left[f_{\tilde{z}\left(l_{2}\right)}^{\dagger}\right]^{q_{2}}$ and 
$\overline{\mathcal{F}}_{q}=\left[f_{\tilde{z}\left(l_{1}^{\prime}\right)}^{\dagger}\right]^{q_{1}}\left[f_{\tilde{z}\left(l_{2}\right)}^{\dagger}\right]^{q_{2}}$ (see Sec. $\left.\mathrm{VB}\right)$. The vacuum expectation values in Eq. (31) thus become

$$
\begin{aligned}
\left\langle 0\left|\mathcal{F}_{q}^{\dagger} \mathcal{F}_{q}\right| 0\right\rangle & =\left\langle 0\left|\overline{\mathcal{F}}_{q}^{\dagger} \overline{\mathcal{F}}_{q}\right| 0\right\rangle=1, \\
\left\langle 0\left|\overline{\mathcal{F}}_{q_{1}=0}^{\dagger}\left(1-i u_{l_{1}, l_{1}^{\prime}} c_{l_{1}} c_{l_{1}^{\prime}}\right) \mathcal{F}_{q_{1}=0}\right| 0\right\rangle & =\left\langle 0\left|\left[1-u_{l_{1}, l_{1}^{\prime}}\left[f_{l_{1}}+f_{l_{1}}^{\dagger}\right]\left[f_{z\left(l_{1}^{\prime}\right)}-f_{z\left(l_{1}^{\prime}\right)}^{\dagger}\right]\right]\right| 0\right\rangle= \begin{cases}2 & \left(\alpha_{l_{1}, l_{1}^{\prime}}=z\right) \\
1 & \left(\alpha_{l_{1}, l_{1}^{\prime}}=x, y\right),\end{cases} \\
\left\langle 0\left|\overline{\mathcal{F}}_{q_{1}=1}^{\dagger}\left(1-i u_{l_{1}, l_{1}^{\prime}} c_{l_{1}} c_{l_{1}^{\prime}}\right) \mathcal{F}_{q_{1}=1}\right| 0\right\rangle & =\left\langle 0\left|f_{z\left(l_{1}^{\prime}\right)}\left[1-u_{l_{1}, l_{1}^{\prime}}\left[f_{l_{1}}+f_{l_{1}}^{\dagger}\right]\left[f_{z\left(l_{1}^{\prime}\right)}-f_{z\left(l_{1}^{\prime}\right)}^{\dagger}\right]\right] f_{l_{1}}^{\dagger}\right| 0\right\rangle= \begin{cases}0 & \left(\alpha_{l_{1}, l_{1}^{\prime}}=z\right) \\
-u_{l_{1}, l_{1}^{\prime}} & \left(\alpha_{l_{1}, l_{1}^{\prime}}=x, y\right),\end{cases}
\end{aligned}
$$

and the corresponding hopping matrix elements take the form

$$
\begin{aligned}
& \tilde{T}_{h, q_{1}=0}= \begin{cases}-t & \left(\alpha_{l_{1}, l_{1}^{\prime}}=z\right) \\
-t / 2 & \left(\alpha_{l_{1}, l_{1}^{\prime}}=x, y\right),\end{cases} \\
& \tilde{T}_{h, q_{1}=1}= \begin{cases}0 & \left(\alpha_{l_{1}, l_{1}^{\prime}}=z\right) \\
u_{l_{1}, l_{1}^{\prime}} t / 2 & \left(\alpha_{l_{1}, l_{1}^{\prime}}=x, y\right) .\end{cases}
\end{aligned}
$$

Note that $u_{l_{1}, l_{1}^{\prime}}=+1$ for $\alpha_{l_{1}, l_{1}^{\prime}}=z$ because $\mathcal{X}_{h}$ excites only $x$ and $y$ bond fermions. Furthermore, the matrix elements in Eq. (33) are independent of the quantum number $q_{2}$. Since the two holes are isolated, the hole hopping between $l_{1}$ and $l_{1}^{\prime}$ is not affected by the other hole at $l_{2}$.

It is crucial to emphasize that the matrix elements in Eq. (33) are valid only if the bond fermion sector is the same before and after the hopping. However, we demonstrate in the following that the hopping problem for a single isolated hole with quantum numbers $h=\{0,1\}$ and $q=\{0,1\}$ can be constructed by referring to these matrix elements only. The most important steps of the construction are illustrated in Fig. 7 , while the resulting hopping problems for the different quantum numbers are summarized in Fig. 8

For a hole with no flux bound to it $(h=0)$, the bond fermion sector is always trivial, and the matrix elements in Eq. (33) are therefore directly applicable. This means that hopping along $x$ and $y$ bonds is allowed for both values of the quantum number $q$, while hopping along $z$ bonds is allowed for $q=0$ but not for $q=1$. In other words, $q=0$ holes can hop in both the $X$ and the $Y$ directions, while $q=1$ holes can hop only in the $X$ direction. Since $u_{l, \alpha(l)}=+1$ for all bonds around an $h=0$ hole, the hopping problem in the $X$ direction is in fact the same for $q=0$ and $q=1$. Note that the opposite sign in the matrix element $\tilde{T}_{h, q_{1}=1}$ is irrelevant because the honeycomb lattice is bipartite.

For a hole with a flux bound to it $(h=1)$, the hopping problem is more complicated because the bond fermion sector depends on the hole position. However, if the hole hops only around the excited plaquette, the bond fermion sector can be chosen to remain the same, and the matrix elements in Eq. (33) are therefore applicable. Remember that the excited plaquette is in an even stripe for a $p=0$ hole (see Fig. (4). Furthermore, the excited plaquette can be shifted along its stripe by applying two simultaneous gauge transformations $D_{l}$ and $\sigma_{l}^{z}$ at the hole site $l$. After these transformations illustrated in Fig. 7 ( a), the bond fermion and the matter fermion sectors around the hole site look the same from the point of view of the new plaquette as they did before from the point of view of the old plaquette. This implies that the hopping problem for $h=1$ is identical to that for $h=0$ as long as the hole hops only around the plaquettes of one particular even stripe. On the other hand, the excited plaquette can only be shifted into a neighboring odd stripe by applying the gauge transformation $\sigma_{l}^{z}$ along with the transformations $c_{\tilde{z}(l)}$ and $i b_{l}^{x} b_{l}^{z}$ that switch the quantum numbers $q$ and $p$. After these transformations illustrated in Fig.7 b), the bond fermion and the matter fermion sectors around the hole site $l$ look the same from the point of view of the new stripe as they did before from the point of view of the old stripe. Since the hopping is independent of $p$, this implies that the hopping problem for $q=1$ around the plaquettes of odd (even) stripes is identical to that for $q=0$ around the plaquettes of even (odd) stripes. Unlike in the case of $h=0$, holes with different values of $q$ do not have fundamentally different hopping problems in the case of $h=1$ : they can both hop along $x$ and $y$ bonds in the $X$ direction, while hopping along $z$ bonds in the $Y$ direction is allowed for $q=0$ in even stripes and for $q=1$ in odd stripes.

\section{Hopping in the gapped phase}

We are now ready to discuss the hopping problem for a single isolated hole at a generic point of the gapped phase away from the isolated dimer limit $(J>0)$. From a perturbation theory in $J \ll 1$, there are possible corrections to the matrix elements in Eq. (33), and the importance of these corrections depends on whether the original matrix element is zero or nonzero. If there is a finite matrix element at $J=0$, the perturbative corrections can be neglected as they only renormalize the matrix element. However, if the matrix element vanishes at $J=0$, these corrections are extremely important as they determine the matrix element in the lowest order.

According to Eq. (33), the only vanishing matrix elements in the isolated dimer limit are $\tilde{T}_{h, q_{1}=1}$ along $z$ bonds. Any such matrix element is zero because the matter fermion corresponding to the two sites $l_{1}$ and $l_{1}^{\prime}$ connected by the $z$ bond is excited: $-i c_{l_{1}} c_{l_{1}^{\prime}}=-1$. To obtain a non-zero correction for the matrix element, we need to find corrections with a nonzero overlap for the ground states before and after the hopping such that $-i c_{l_{1}} c_{l_{1}^{\prime}}=+1$ for both corrections. In general, these two corrections belong to two complementary sections 
(a)

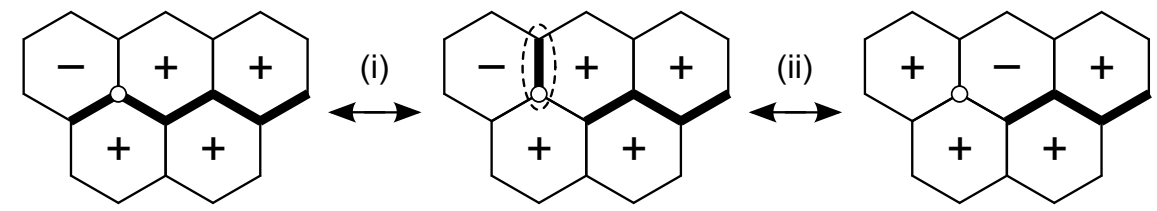

(b)

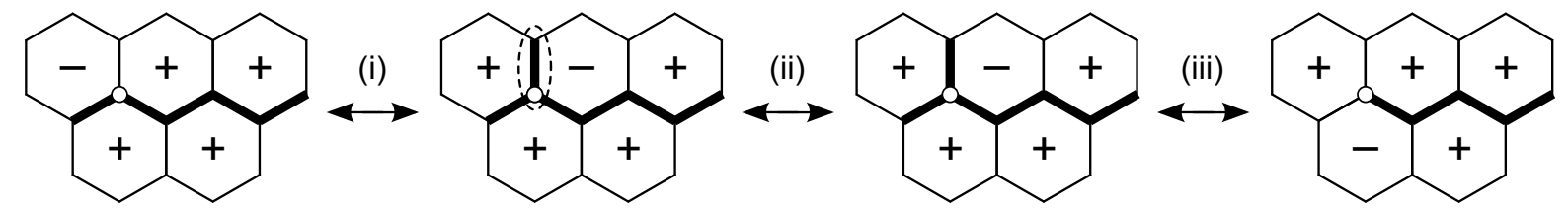

FIG. 7: Different types of transformations relating bond fermion and matter fermion sectors around a hole site $l \in \Delta$ (white dot) when there is a flux bound to the hole. Each bond fermion sector is labeled with the excited bond fermions (thick lines) and the corresponding plaquette operator eigenvalues $( \pm 1)$, while each matter fermion sector is labeled with the excited matter fermions (dashed ellipses). (a) Gauge transformations $D_{l}$ (i) and $\sigma_{l}^{z}$ (ii) for shifting the excited plaquette within a stripe. (b) Transformations $\sigma_{l}^{z}$ (i) [gauge], $c_{\tilde{z}(l)}$ (ii) [q-switch], and $i b_{l}^{x} b_{l}^{z}$ (iii) [ $p$-switch] for shifting the excited plaquette between neighboring stripes.

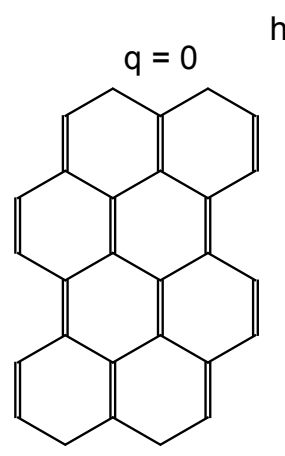

$\mathrm{h}=0$

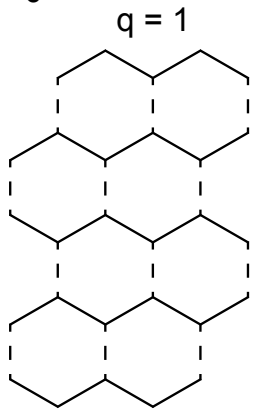

$h=1$

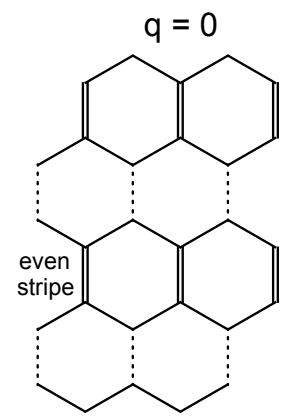

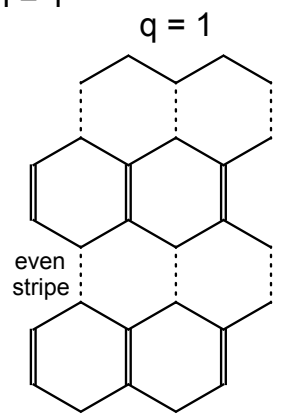

FIG. 8: Hole hopping problems in the isolated dimer limit for different combinations of the flux quantum number $h=\{0,1\}$ and the fermion quantum number $q=\{0,1\}$. Each bond is labeled according to the effective hopping amplitude $T$ along it: double solid lines indicate $T=-t$, single solid lines indicate $T=-t / 2$, while dashed and dotted lines indicate $T=0$. For dashed lines, the effective hopping amplitude vanishes only in the isolated dimer limit, while for dotted lines, it vanishes in the entire gapped phase.

of an open string connecting the sites $l_{1}$ and $l_{1}^{\prime}$. For example, if we use the site labeling convention in Fig. 9 around the sites $l_{1}$ and $l_{1}^{\prime}$, one such pair of corrections is

$$
\begin{aligned}
& \mathcal{F}_{q_{1}=1}|0\rangle=\frac{J}{6}\left(b_{5}^{x} b_{6}^{x} c_{5} c_{6}\right) \frac{J}{4}\left(b_{3}^{x} b_{2}^{x} c_{3} c_{2}\right) f_{1}^{\dagger}|0\rangle, \\
& \overline{\mathcal{F}}_{q_{1}=1}|0\rangle=\frac{J}{6}\left(b_{1}^{y} b_{2}^{y} c_{1} c_{2}\right) \frac{J}{4}\left(b_{5}^{y} b_{4}^{y} c_{5} c_{4}\right) f_{1}^{\dagger}|0\rangle,
\end{aligned}
$$

and the resulting correction to the ground-state overlap is

$$
\begin{aligned}
& \left\langle 0\left|\overline{\mathcal{F}}_{q_{1}=1}^{\dagger}\left(1-i c_{1} c_{6}\right) \mathcal{F}_{q_{1}=1}\right| 0\right\rangle=J^{4} u_{1,2} u_{3,2} u_{5,4} u_{5,6} Z_{P} \\
& =J^{4} W_{P} Z_{P}, \\
& Z_{P}=\frac{1}{576}\left\langle 0\left|f_{1} c_{5} c_{4} c_{1} c_{2}\left(1-i c_{1} c_{6}\right) c_{5} c_{6} c_{3} c_{2} f_{1}^{\dagger}\right| 0\right\rangle \\
& =\frac{1}{288}\left\langle 0\left|f_{1} c_{5} c_{4} c_{1} c_{2} c_{5} c_{6} c_{3} c_{2} f_{1}^{\dagger}\right| 0\right\rangle=\frac{1}{288} \text {. }
\end{aligned}
$$

Note that $u_{1,6}=u_{3,4}=+1$ because $\mathcal{X}_{h}$ is defined such that it excites only $x$ and $y$ bond fermions.

For a generic open string connecting the sites 1 and $1^{\prime}$, we define a closed loop $C$ consisting of the open string and the

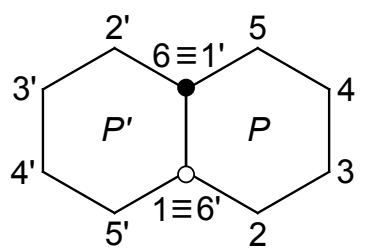

FIG. 9: Site and plaquette labeling conventions around the sites $l_{1}$ (white dot) and $l_{1}^{\prime}$ (black dot) when considering the hopping along the $z$ bond between $l_{1}$ and $l_{1}^{\prime}$.

$z$ bond between 1 and $1^{\prime}$. Any correction to the ground-state overlap due to the open string is then proportional to $W_{C} Z_{C}$, where $W_{C}$ is the corresponding loop operator eigenvalue and $Z_{C}$ is an expectation value similar to that in Eq. (36). By means of a reflection across the middle of the $z$ bond, we also define a dual loop $C^{\prime}$ with a loop operator eigenvalue $W_{C^{\prime}}$ and a dual correction with an expectation value $Z_{C^{\prime}}$. Note that the dual correction strictly corresponds to a backward hopping because the reflection exchanges the sites 1 and $1^{\prime}$. On the other hand, $Z_{C^{\prime}} \in \mathbb{R}$ means that there is an equivalent dual correction for the forward hopping as well. If we identify the 
site labels of the loop $C$ with the dual site labels of the loop $C^{\prime}$, the explicit forms of the expectation values $Z_{C}$ and $Z_{C^{\prime}}$ are identical, and thus $Z_{C}=Z_{C^{\prime}}$. Since this equality is true for all corrections, we conclude that the total corrections due to the loops $C$ and $C^{\prime}$ have equal magnitudes, while their relative signs are determined by the loop eigenvalues $W_{C, C^{\prime}}$.

This result has already strong implications for holes with a flux bound to them $(h=1)$. When the bond fermion sector is conserved by the hopping, the flux is necessarily bound to either of the plaquettes $P$ or $P^{\prime}$. If we then choose any two dual loops $C$ and $C^{\prime}$ that do not enclose any other holes, one of them contains one excited plaquette and the other one contains no excited plaquettes. This implies $W_{C}+W_{C^{\prime}}=0$, and therefore the corrections due to all of the paired-up dual loops vanish because $\left(W_{C}+W_{C^{\prime}}\right) Z_{C}=0$. The only non-zero corrections are then due to loops that are large enough such that they enclose at least one other hole with a flux bound to it. If the smallest distance between any two holes is $R$, the length of such a loop is at least $2 R$, and therefore the lowest- order corrections to the matrix element are $\sim J^{2 R}$. Since this quantity is exponentially small in the $R \gg 1$ limit, holes with $h=1$ can hop only along their respective stripes as long as we are in the gapped phase with $J<1 / 2$.

For holes with no flux bound to them $(h=0)$, there are no excited plaquettes, and all loops have $W_{C}=+1$. This means that the lowest-order corrections to the ground-state overlap are due to the plaquettes $P$ and $P^{\prime}$. These two corrections are identical because $W_{P}=W_{P^{\prime}}=+1$. The total expectation value $Z_{P}$ is obtained by considering all possible ways of dividing the open string between the sites 1 and $1^{\prime}$ into two complementary sections and all possible ways of ordering the $x$ and $y$ bonds within the resulting two sections. Note that the choice of the complementary sections is limited by the fact that some bonds have switched-off interactions: the bonds around the site 1 can only be used after the hopping, while the bonds around the site $1^{\prime}$ can only be used before the hopping. Exploiting symmetry to reduce the number of inequivalent terms, the total expectation value becomes

$$
\begin{aligned}
Z_{P}= & \frac{2}{16}\left\langle 0\left|f_{1} c_{1} c_{2} \epsilon_{1,6} c_{3} c_{2} c_{5} c_{4} c_{5} c_{6} f_{1}^{\dagger}\right| 0\right\rangle+\frac{2}{48}\left\langle 0\left|f_{1} c_{1} c_{2} \epsilon_{1,6} c_{5} c_{4} c_{3} c_{2} c_{5} c_{6} f_{1}^{\dagger}\right| 0\right\rangle+\frac{2}{32}\left\langle 0\left|f_{1} c_{1} c_{2} \epsilon_{1,6} c_{3} c_{2} c_{5} c_{6} c_{5} c_{4} f_{1}^{\dagger}\right| 0\right\rangle \\
& +\frac{2}{64}\left\langle 0\left|f_{1} c_{1} c_{2} \epsilon_{1,6} c_{5} c_{6} c_{3} c_{2} c_{5} c_{4} f_{1}^{\dagger}\right| 0\right\rangle+\frac{2}{96}\left\langle 0\left|f_{1} c_{1} c_{2} \epsilon_{1,6} c_{5} c_{4} c_{5} c_{6} c_{3} c_{2} f_{1}^{\dagger}\right| 0\right\rangle+\frac{2}{64}\left\langle 0\left|f_{1} c_{1} c_{2} \epsilon_{1,6} c_{5} c_{6} c_{5} c_{4} c_{3} c_{2} f_{1}^{\dagger}\right| 0\right\rangle \\
& +\frac{1}{16}\left\langle 0\left|f_{1} c_{1} c_{2} c_{3} c_{2} \epsilon_{1,6} c_{5} c_{4} c_{5} c_{6} f_{1}^{\dagger}\right| 0\right\rangle+\frac{2}{32}\left\langle 0\left|f_{1} c_{1} c_{2} c_{3} c_{2} \epsilon_{1,6} c_{5} c_{6} c_{5} c_{4} f_{1}^{\dagger}\right| 0\right\rangle+\frac{1}{64}\left\langle 0\left|f_{1} c_{3} c_{2} c_{1} c_{2} \epsilon_{1,6} c_{5} c_{6} c_{5} c_{4} f_{1}^{\dagger}\right| 0\right\rangle \\
& +\frac{1}{144}\left\langle 0\left|f_{1} c_{1} c_{2} c_{5} c_{4} \epsilon_{1,6} c_{3} c_{2} c_{5} c_{6} f_{1}^{\dagger}\right| 0\right\rangle+\frac{2}{288}\left\langle 0\left|f_{1} c_{1} c_{2} c_{5} c_{4} \epsilon_{1,6} c_{5} c_{6} c_{3} c_{2} f_{1}^{\dagger}\right| 0\right\rangle+\frac{1}{576}\left\langle 0\left|f_{1} c_{5} c_{4} c_{1} c_{2} \epsilon_{1,6} c_{5} c_{6} c_{3} c_{2} f_{1}^{\dagger}\right| 0\right\rangle \\
= & \frac{1}{4}+\frac{1}{12}-\frac{1}{8}-\frac{1}{16}+\frac{1}{24}-\frac{1}{16}+\frac{1}{8}-\frac{1}{8}+\frac{1}{32}+\frac{1}{72}+\frac{1}{72}+\frac{1}{288}=\frac{3}{16},
\end{aligned}
$$

where $\epsilon_{1,6} \equiv 1-i c_{1} c_{6}=2$ in all the terms above. Since $W_{P}=W_{P^{\prime}}=+1$ and $Z_{P}=Z_{P^{\prime}}=3 / 16$, the corresponding lowest-order correction to the ground-state overlap is $\left\langle 0\left|\overline{\mathcal{F}}_{q_{1}=1}^{\dagger} \epsilon_{1,6} \mathcal{F}_{q_{1}=1}\right| 0\right\rangle=3 J^{4} / 8$. On the other hand, the ground-state norms $\left\langle 0\left|\mathcal{F}_{q}^{\dagger} \mathcal{F}_{q}\right| 0\right\rangle$ and $\left\langle 0\left|\overline{\mathcal{F}}_{q}^{\dagger} \overline{\mathcal{F}}_{q}\right| 0\right\rangle$ are still approximately 1 , and therefore the lowest-order correction to the hopping matrix element takes the form

$$
\tilde{T}_{h_{1}=0, q_{1}=1}=-\frac{3}{16} J^{4} t \quad\left(\alpha_{l_{1}, l_{1}^{\prime}}=z\right) .
$$

This result shows that holes with $h=0$ and $q=1$ are only confined to hop in the $X$ direction in the limit of $J \rightarrow 0$. At a generic point of the gapped phase, holes with $h=0$ are free to hop in both the $X$ and the $Y$ directions.

It is instructive to investigate the hole hopping problems across the entire gapped phase with $0<J<1 / 2$. Since the perturbation theory in $J \ll 1$ is not applicable in general, we need to evaluate the hopping matrix elements in Eq. (31) numerically. The resulting hopping matrix elements for quantum numbers $h=0$ and $q=\{0,1\}$ are plotted across the gapped phase in Fig. 10 In the limit of $J \rightarrow 0$, when the perturba- tion theory is valid, the hopping matrix elements in Eqs. 33. and (38) are accurately recovered. In the opposite limit of $J \rightarrow 1 / 2$, when the phase transition to the gapless phase is close, the hopping matrix elements for all quantum numbers $h=\{0,1\}$ and $q=\{0,1\}$ become strongly dependent on the system size and exhibit a sudden drop towards zero. These results are both explained by the vanishing energy gap of the bulk fermion excitations: finite-size effects become important due to the divergent correlation length, while the hopping matrix elements vanish due to the hybridization between the hole fermions and the lowest-energy bulk fermions.

Note that the condition of slow hopping breaks down in the limit of $J \rightarrow 1 / 2$ as the lowest-energy bulk fermions no longer have finite excitation energies. The hopping process in this limit involves not only the respective ground states as in Sec. VIA but also the excited states in which some of the lowest-energy bulk fermions are excited. On the other hand, this means that the hopping matrix elements in Eq. 31) underestimate the actual hopping amplitudes, and therefore the vanishing hopping matrix elements at $J \rightarrow 1 / 2$ do not imply that the holes become stationary at the phase transition point. 

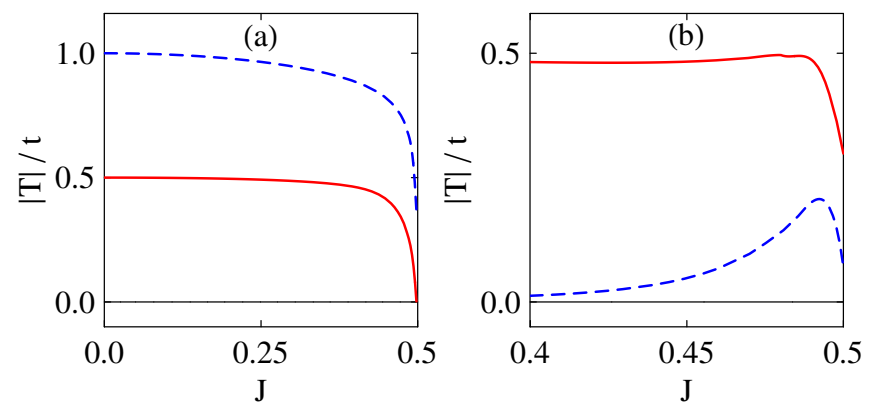

FIG. 10: (Color online) Effective hopping amplitude as a function of the $x$ and $y$ bond coupling strengths within the gapped phase for a hole with $h=0$ and either $q=0$ (a) or $q=1$ (b) along $x$ and $y$ bonds (solid lines) and along $z$ bonds (dashed lines). The lattice dimensions are $N_{X}=N_{Y}=20$ in all cases.

\section{E. Particle statistics}

Since the quantum numbers $h, q$, and $p$ are conserved by the hopping process, we can treat holes with different quantum numbers as distinct particles and determine their respective particle statistics. To this end, we consider an exchange process in which two isolated identical holes at sites 0 and $\ell$ are exchanged along a closed loop $C$ that contains $L$ sites labeled $\{1,2, \ldots, \ell-1, \ell, \ell+1, \ldots, L \equiv 0\}$. If the exchange process is adiabatically slow, the final state is identical to the initial state up to a complex phase factor $\exp (i \varphi)$. The corresponding phase $\varphi$ has two contributions: a dynamic phase from the time integral of the governing Hamiltonian that depends on the details of the exchange process, and a geometric phase $\theta_{2}$ that depends only on the loop $C$. To determine the particle statistics, we first need to obtain the phase $\theta_{2}$.

The adiabatic exchange process along the loop $C$ starts from the initial ground state $\left|\Omega_{h, q, p}^{\{0, \ell\}}\right\rangle$, ends at the exchanged ground state $\left|\Omega_{h, q, p}^{\{\ell, 0\}}\right\rangle$, and happens via subsequent nearestneighbor hopping processes through intermediate ground states $\left|\Omega_{h, q, p}^{\left\{l, l^{\prime}\right\}}\right\rangle$, where $0 \leq l \leq \ell$ and $\ell \leq l^{\prime} \leq L$. These hopping processes are illustrated in Fig. 11 a). The geometric phase $\theta_{2}$ arises from the geometric connections between the intermediate ground states $\left|\Omega_{h, q, p}^{\left\{l, l^{\prime}\right\}}\right\rangle$. On the other hand, it can be argued theoretically and verified numerically that these geometric connections are given by the hopping matrix elements in Eq. 287. Since there is exactly one intermediate hopping process for each section of the loop, this suggests that the phase $\theta_{2}$ is the phase of the product of all the hopping matrix elements around the loop $C$. In fact, we need to consider two additional phase factors due to the two holes being exchanged. First, the exchanged ground state $\left|\Omega_{h, q, p}^{\{\ell, 0\}}\right\rangle$ can contain a non-trivial phase factor with respect to the initial ground state $\left|\Omega_{h, q, p}^{\{0, \ell\}}\right\rangle$. Second, our hopping formalism in the hole spin picture ignores the inherent fermionic nature of the holes. Since the two hole spins are removed from both ground states $\left|\Omega_{h, q, p}^{\{0, \ell\}}\right\rangle$ and $\left|\Omega_{h, q, p}^{\{\ell, 0\}}\right\rangle$ by fermionic annihilation operators, the exchange between the two holes corresponds to a non-trivial phase factor -1 in the actual hole picture. The (a)

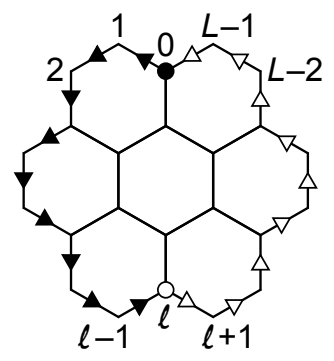

(b)

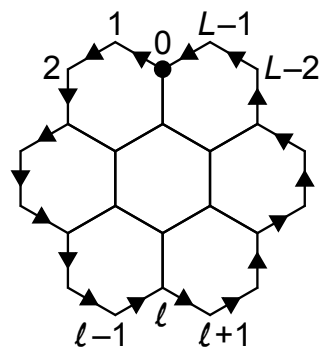

FIG. 11: Illustrations of the two processes that are used to evaluate the statistical angle $\vartheta=\theta_{2}-\theta_{1}$. (a) Exchange process for obtaining $\theta_{2}$. Two identical holes with the given quantum numbers at sites 0 and $\ell=9$ (black and white dots) are exchanged along a closed loop of length $L=18$. The subsequent hopping processes for the respective holes are marked by black and white arrows. (b) Looping process for obtaining $\theta_{1}$. One hole with the same quantum numbers at site 0 (black dot) is moved around the same closed loop. The subsequent hopping processes are marked by black arrows.

geometric phase $\theta_{2}$ thus takes the form

$$
\begin{gathered}
\theta_{2}=\arg \left[-\left\langle\Omega_{h, q, p}^{\{0, \ell\}} \mid \Omega_{h, q, p}^{\{\ell, 0\}}\right\rangle \prod_{l=0}^{L-1} \mathrm{~T}_{l, l+1}^{(2)}\right], \\
\mathrm{T}_{l, l+1}^{(2)}=-\frac{t\left\langle\Omega_{h, q, p}^{\left\{l+1, l^{\prime}\right\}}\left|\mathcal{E}_{l, l+1}\right| \Omega_{h, q, p}^{\left\{l, l^{\prime}\right\}}\right\rangle}{\sqrt{\left\langle\Omega_{h, q, p}^{\left\{l+1, l^{\prime}\right\}} \mid \Omega_{h, q, p}^{\left\{l+1, l^{\prime}\right\}}\right\rangle\left\langle\Omega_{h, q, p}^{\left\{l, l^{\prime}\right\}} \mid \Omega_{h, q, p}^{\left\{l, l^{\prime}\right\}}\right\rangle}} .
\end{gathered}
$$

Note that the matrix element $\mathrm{T}_{l, l+1}^{(2)}$ does not depend on the site $l^{\prime}$ of the other hole as the two holes are assumed to be isolated at each step of the exchange process.

The geometric phase of the exchange process can be written as a sum of two terms: $\theta_{2}=\vartheta+\theta_{1}$. The first term $\vartheta$ is the actual statistical phase that specifies the particle statistics, while the second term $\theta_{1}$ is the geometric (Berry) phase of a looping process in which a single hole at site 0 with the same quantum numbers $h, q$, and $p$ is moved adiabatically slowly around the same closed loop $C$. Since the statistical angle is given by $\vartheta=\theta_{2}-\theta_{1}$ in terms of the two geometric phases, we also need to obtain the second phase $\theta_{1}$.

The adiabatic looping process around the loop $C$ starts from the initial ground state $\left|\Omega_{h, q, p}^{\{0\}}\right\rangle$, ends at the final ground state $\left|\Omega_{h, q, p}^{\{L\}}\right\rangle \equiv\left|\Omega_{h, q, p}^{\{0\}}\right\rangle$, and happens via subsequent nearest-neighbor hopping processes through intermediate ground states $\left|\Omega_{h, q, p}^{\{l\}}\right\rangle$, where $0<l<L$. These hopping processes are illustrated in Fig. 11 b). As in the case of the exchange process, the geometric phase $\theta_{1}$ of the looping process arises from the geometric connections between the intermediate ground states, and is therefore related to the product of the hopping matrix elements around the loop $C$. However, the two additional phase factors are absent because no holes are being exchanged. The geometric phase $\theta_{1}$ thus takes the form

$$
\theta_{1}=\arg \left[\prod_{l=0}^{L-1} \mathrm{~T}_{l, l+1}^{(1)}\right]
$$




$$
\mathrm{T}_{l, l+1}^{(1)}=-\frac{t\left\langle\Omega_{h, q, p}^{\{l+1\}}\left|\mathcal{E}_{l, l+1}\right| \Omega_{h, q, p}^{\{l\}}\right\rangle}{\sqrt{\left\langle\Omega_{h, q, p}^{\{l+1\}} \mid \Omega_{h, q, p}^{\{l+1\}}\right\rangle\left\langle\Omega_{h, q, p}^{\{l\}} \mid \Omega_{h, q, p}^{\{l\}}\right\rangle}} .
$$

Importantly, the matrix element $\mathrm{T}_{l, l+1}^{(1)}$ is in most cases identical to the matrix element $\mathrm{T}_{l, l+1}^{(2)}$ because the presence of the other isolated hole is irrelevant. The only exception is the case of $h=1$ and $q=1$ when there is a string of excited bond fermions connected to the hole and the hopping is sensitive to excited bond fermions [see Eq. [33)]. It is then relevant for at least one section of the loop $C$ whether the other end of the string is at the other hole moving around the same loop or at a stationary hole in the background.

We are now ready to determine the particle statistics of the various hole types. From a direct comparison between Eqs. (39) and 41, the statistical phase becomes

$$
\vartheta=\arg \left[-\left\langle\Omega_{h, q, p}^{\{0, \ell\}} \mid \Omega_{h, q, p}^{\{\ell, 0\}}\right\rangle \prod_{l=0}^{L-1}\left(\frac{\mathrm{T}_{l, l+1}^{(2)}}{\mathrm{T}_{l, l+1}^{(1)}}\right)\right] .
$$

Furthermore, if the holes have quantum numbers other than $h=1$ and $q=1$, the matrix elements $\mathrm{T}_{l, l+1}^{(1)}$ and $\mathrm{T}_{l, l+1}^{(2)}$ are identical, and therefore Eq. (43) reduces to

$$
\vartheta=\arg \left[-\left\langle\Omega_{h, q, p}^{\{0, \ell\}} \mid \Omega_{h, q, p}^{\{\ell, 0\}}\right\rangle\right] .
$$

By evaluating $\vartheta$ for all hole types, we can then directly obtain their particle statistics: $\vartheta=0$ is indicative of bosons, while $\vartheta=\pi$ is indicative of fermions.

For holes with $h=0$ and $q=0$, the initial ground state $\left|\Omega_{0,0, p}^{\{0, \ell\}}\right\rangle$ and the final ground state $\left|\Omega_{0,0, p}^{\{\ell, 0\}}\right\rangle$ are identical by construction. In the isolated dimer limit, the two ground states for $p=0$ holes are $\left|\Omega_{0,0,0}^{\{0, \ell\}}\right\rangle=\left|\Omega_{0,0,0}^{\{\ell, 0\}}\right\rangle=\mathcal{Q}_{\{0, \ell\}} \mathcal{P}|0\rangle$. In the general case, there are additional operators $B_{p} \neq 1$ and $\mathcal{F}_{0 ; 0} \neq 1$ that set the bond fermion and the matter fermion sectors. On the other hand, these operators are the same for both ground states, and therefore the relation $\left|\Omega_{0,0, p}^{\{0, \ell\}}\right\rangle=\left|\Omega_{0,0, p}^{\{\ell, 0\}}\right\rangle$ remains true. Since applying Eq. (44) then gives $\vartheta=\pi$, we conclude that holes with $h=0$ and $q=0$ are fermions.

For holes with $h=0$ and $q=1$, the initial ground state $\left|\Omega_{0,1, p}^{\{0, \ell\}}\right\rangle$ and the final ground state $\left|\Omega_{0,1, p}^{\{\ell, 0\}}\right\rangle$ are only identical up to a minus sign as the two ground states have the two hole fermions at sites 0 and $\ell$ excited in an opposite order. In the isolated dimer limit, the two ground states for $p=0$ holes are $\left|\Omega_{0,1,0}^{\{0, \ell\}}\right\rangle=-\left|\Omega_{0,1,0}^{\{\ell, 0\}}\right\rangle=\mathcal{Q}_{\{0, \ell\}} \mathcal{P} f_{\tilde{z}(0)}^{\dagger} f_{\tilde{z}(\ell)}^{\dagger}|0\rangle$. In the general case, there are additional operators $B_{p} \neq 1$ and $\mathcal{F}_{1 ; 0} \neq 1$ that set the bond fermion and the matter fermion sectors. On the other hand, these operators are the same for both ground states, and therefore the relation $\left|\Omega_{0,1, p}^{\{0, \ell\}}\right\rangle=-\left|\Omega_{0,1, p}^{\{\ell, 0\}}\right\rangle$ remains true. Since applying Eq. (44) then gives $\vartheta=0$, we conclude that holes with $h=0$ and $q=1$ are bosons.

It is crucial that holes with $h=1$ can move only around the plaquettes of particular stripes: even stripes for $q=0$ and odd stripes for $q=1$. Furthermore, it is shown by Fig. 7 that $q=0$ holes in even stripes are equivalent to $q=1$ holes in odd stripes. This implies that these two hole types have the same particle statistics, and therefore it is enough to consider one of them. We choose to consider holes with $h=1$ and $q=0$ because Eq. (44) is then applicable. For these holes, the only difference in the ground state with respect to holes with $h=0$ and $q=0$ is the presence of an additional flux-binding operator $\mathcal{X}_{1} \neq 1$. On the other hand, this operator is the same for the initial and the final ground states, and therefore the two ground states are identical: $\left|\Omega_{1,0, p}^{\{0, \ell\}}\right\rangle=\left|\Omega_{1,0, p}^{\{\ell, 0\}}\right\rangle$. Since applying Eq. (44) then gives $\vartheta=\pi$, we conclude that holes with $h=1$ and $q=\{0,1\}$ are fermions.

To supplement the above derivations, we also provide an intuitive explanation for the particle statistics found. The main principle is that the holes in the model can bind the elementary excitations of the model: fluxes and fermions. The various hole types with different quantum numbers $h$ and $q$ are then distinguished only by the kinds of elementary excitations that are bound to them. In particular, a hole with a non-trivial flux quantum number $h=1$ has a bound flux, while a hole with a non-trivial fermion quantum number $q=1$ has a bound fermion. Holes with $h=0$ and $q=0$ are interpreted as bare holes with no elementary excitations bound to them. Since bare holes are missing spin one-half fermions, it is natural that they are fermions themselves. Conversely, the remaining three types of holes are interpreted as composite holes made out of bare holes and elementary excitations. Due to the presence of the bound excitations, their statistics can be different from that of bare holes. For holes with $h=0$ and $q=1$, the binding of a fermion leads to a statistical transmutation, and therefore these holes are bosons. For holes with $h=1$ and $q=0$, the binding of a flux has no effect on the statistics, and therefore these holes are fermions. We might then naively expect that holes with $h=1$ and $q=1$ should be bosons because there is a statistical transmutation due to the binding of a fermion. However, the bound flux and the bound fermion have semionic relative statistics. Since this corresponds to an additional transmutation for the composite hole, these holes are in fact fermions. The particle statistics of the various hole types along with their interpretations in terms of the bound excitations are summarized in Table VI

\begin{tabular}{c|c|c|c}
\hline \hline \multicolumn{2}{c|}{ Hole type } & Statistics & Interpretation \\
\hline \multirow{2}{*}{$h=0$} & $q=0$ & Fermion & Bare hole \\
& $q=1$ & Boson & Hole + fermion \\
\hline \multirow{2}{*}{$h=1$} & $q=0$ & Fermion & Hole + flux \\
& $q=1$ & Fermion & Hole + flux + fermion \\
\hline \hline
\end{tabular}

TABLE VI: Absolute statistics of holes with flux quantum numbers $h=\{0,1\}$ and fermion quantum numbers $q=\{0,1\}$ from a process when two identical holes are exchanged. Interpretations are given in terms of elementary excitations bound.

It is also useful to investigate the relative statistics between the various hole types. To this end, we consider two looping processes in which a hole with quantum numbers $h$ and $q$ is moved around a closed loop $C$. In the first case, there is no hole enclosed by the loop, and Eq. 411 gives a geometric phase $\theta_{1}$. In the second case, there is one stationary hole with quantum numbers $h^{\prime}$ and $q^{\prime}$ enclosed by the loop, and 
Eq. (41) gives a geometric phase $\theta_{1}^{\prime}$. The relative statistics between holes with quantum numbers $h$ and $q$ and holes with quantum numbers $h^{\prime}$ and $q^{\prime}$ is then specified by the relative statistical phase $\vartheta^{\prime}=\theta_{1}^{\prime}-\theta_{1}$. For $\vartheta^{\prime}=0$, the two hole types have trivial relative statistics, while for $\vartheta^{\prime} \neq 0$, the two hole types have anyonic relative statistics. Importantly, the relative statistical phase $\vartheta^{\prime}$ is symmetric in the two hole types: it does not depend on which one is kept stationary and which one is moved around the loop.

We first notice that the two looping processes giving the phases $\theta_{1}$ and $\theta_{1}^{\prime}$ are not both possible for all combinations of the quantum numbers. For mobile holes with $h=1$, the exchange process and the looping process with no hole enclosed are barely possible, but the looping process with a stationary hole enclosed is impossible. Since these holes can move only around the plaquettes of particular stripes, there is no space for a stationary hole inside any loop they can possibly move around. This means that the mobile hole must have a trivial flux quantum number $h=0$. On the other hand, the stationary hole can then only influence the hopping of the mobile hole if the stationary hole is connected to a string of excited bond fermions and the hopping of the mobile hole is sensitive to excited bond fermions. This corresponds to quantum numbers $h^{\prime}=1$ and $q^{\prime}=\{0,1\}$ for the stationary hole and quantum numbers $h=0$ and $q=1$ for the mobile hole. In these cases, one hopping matrix element picks up a minus sign at the intersection point of the loop and the string of excited bond fermions [see Eq. [33)]. This implies that the relative statistical phase is $\vartheta^{\prime}=\pi$, and therefore the two hole types have semionic relative statistics. In all other possible cases, the hopping of the mobile hole is not influenced by the stationary hole. This implies that the relative statistical phase is $\vartheta^{\prime}=0$, and therefore the two hole types have trivial relative statistics. The results for the relative statistics between the various hole types are summarized in Table VII

\begin{tabular}{c|c|cc|cc}
\hline \hline \multicolumn{2}{c|}{ Hole type } & \multicolumn{2}{c|}{$h^{\prime}=0$} & \multicolumn{2}{c}{$h^{\prime}=1$} \\
\cline { 3 - 6 } \multicolumn{2}{c}{} & $q^{\prime}=0$ & $q^{\prime}=1$ & $q^{\prime}=0$ & $q^{\prime}=1$ \\
\hline \multirow{2}{*}{$h=0$} & $q=0$ & 0 & 0 & 0 & 0 \\
& $q=1$ & 0 & 0 & $\pi$ & $\pi$ \\
\hline \multirow{2}{*}{$h=1$} & $q=0$ & - & - & - & - \\
& $q=1$ & - & - & - & - \\
\hline \hline
\end{tabular}

TABLE VII: Relative statistics between holes with quantum numbers $h$ and $q$ and holes with quantum numbers $h^{\prime}$ and $q^{\prime}$ from a process when the former hole type is moved around the latter hole type: $\vartheta^{\prime}=0$ indicates trivial statistics, $\vartheta^{\prime}=\pi$ indicates semionic statistics, while there is no value if the process is impossible.

We can also interpret the relative statistics in terms of the elementary excitations bound to the holes. First, two bare holes or two identical elementary excitations have trivial relative statistics. Second, the relative statistics between a bare hole and an elementary excitation is trivial, while that between a flux and a fermion is semionic. As a result of these properties, the relative statistics between two identical holes and that between a bare hole and a composite hole is trivial, while that between two distinct composite holes is semionic. The entries of Table VII can then be obtained, even the ones that correspond to impossible processes: the diagonal entries and the entries of the first row or the first column are $\vartheta^{\prime}=0$, while the remaining entries are $\vartheta^{\prime}=\pi$. We finally remark that all of our results for the absolute and the relative particle statistics are consistent with the correspondence between the various hole types and the superselection sectors (see Table $\mathrm{V}$.

\section{FINITE DENSITY OF MOBILE HOLES}

\section{A. Non-interacting treatment}

We now consider the Kitaev honeycomb model with a finite density of mobile holes. The hole density $\rho=n / 2 N$ gives the fraction of sites $l$ that are hole sites $l \in \Delta$. For simplicity, we assume a small hole density $\rho \ll 1$ and neglect any hole interactions. The ground state of the model is then a multi-hole state of $n$ non-interacting holes: depending on their particle statistics, these holes either form a Bose condensate or fill up a Fermi sea. By evaluating the multi-hole energy for all combinations of $h=\{0,1\}$ and $q=\{0,1\}$, we can determine the ground-state quantum numbers.

The most straightforward way to represent the multi-hole state is to use appropriate single-hole creation and annihilation operators. If the operator $a_{h, q, p}^{(\dagger)}\left(\mathbf{R}_{l}\right)$ annihilates (creates) a hole at site $l$ with quantum numbers $h, q$, and $p$, the multi-hole state of $n$ stationary holes at sites $\Delta=\left\{l_{j}\right\}$ with quantum numbers $\left\{h_{j}\right\},\left\{q_{j}\right\}$, and $\left\{p_{j}\right\}$ reads as

$$
\left|\Omega_{h, q, p}^{\Delta}\right\rangle=\prod_{j=1}^{n} a_{h_{j}, q_{j}, p_{j}}^{\dagger}\left(\mathbf{R}_{l_{j}}\right)|\Omega\rangle
$$

where $|\Omega\rangle$ is the ground state of the model with no holes, and the lattice position $\mathbf{R}_{l}=\left(X_{l}, Y_{l}\right)$ of the site $l$ is measured in units of the lattice constant. We now assume and later verify that holes with distinct flux quantum numbers $h=\{0,1\}$ are not simultaneously present in this multi-hole state. Since Table VII shows that no anyonic relative statistics manifests itself between holes with identical flux quantum numbers, the single-hole operators $a_{h, q, p}^{(\dagger)}\left(\mathbf{R}_{l}\right)$ can then be treated as standard bosonic and fermionic operators. In particular, they satisfy bosonic commutation relations in the case of $h=0$ and $q=1$, and fermionic anticommutation relations in all other cases, except for an overall hard-core constraint that there can be at most one hole of any type at each site. However, if the hole density $\rho$ is sufficiently small, this hard-core constraint is practically irrelevant. We can then write an effective Hamiltonian for the model with $n$ mobile holes in terms of the standard bosonic and fermionic operators $a_{h, q, p}^{(\dagger)}\left(\mathbf{R}_{l}\right)$. In the absence of hole interactions, this Hamiltonian is quadratic: it contains an onsite potential term corresponding to the flux-binding energy discussed in Sec. $\mathrm{VB}$ and several hopping terms corresponding to the hopping problems in Fig. 8 Taking the isolated dimer limit and keeping only the lowest-order terms in $J \ll 1$, the effective Hamiltonian takes the form 


$$
\begin{aligned}
H_{a}= & \Gamma_{0}-\frac{9 J^{8}}{1024} \sum_{l} \sum_{q, p} \hat{n}_{1, q, p}\left(\mathbf{R}_{l}\right)-\frac{t}{2} \sum_{l \in A} \sum_{\alpha=x, y} \sum_{h, q, p}\left[a_{h, q, p}^{\dagger}\left(\mathbf{R}_{l}\right) a_{h, q, p}\left(\mathbf{R}_{\alpha(l)}\right)+\text { H.c. }\right] \\
& -t \sum_{l \in A} \sum_{p}\left[a_{0,0, p}^{\dagger}\left(\mathbf{R}_{l}\right) a_{0,0, p}\left(\mathbf{R}_{z(l)}\right)+\text { H.c. }\right]-\frac{3 J^{4} t}{16} \sum_{l \in A} \sum_{p}\left[a_{0,1, p}^{\dagger}\left(\mathbf{R}_{l}\right) a_{0,1, p}\left(\mathbf{R}_{z(l)}\right)+\text { H.c. }\right] \\
& -t \sum_{l \in A^{\prime}} \sum_{p}\left[a_{1,0, p}^{\dagger}\left(\mathbf{R}_{l}\right) a_{1,0, p}\left(\mathbf{R}_{z(l)}\right)+\text { H.c. }\right]-t \sum_{l \in A^{\prime \prime}} \sum_{p}\left[a_{1,1, p}^{\dagger}\left(\mathbf{R}_{l}\right) a_{1,1, p}\left(\mathbf{R}_{z(l)}\right)+\text { H.c. }\right],
\end{aligned}
$$

where $\Gamma_{0}$ is the ground-state energy of the model with $n$ stationary $h=0$ holes, and $\hat{n}_{h, q, p}\left(\mathbf{R}_{l}\right) \equiv a_{h, q, p}^{\dagger}\left(\mathbf{R}_{l}\right) a_{h, q, p}\left(\mathbf{R}_{l}\right)$ is the number operator. The fixed total number of holes is enforced by the constraint $n=\sum_{l} \sum_{h, q, p}\left\langle\hat{n}_{h, q, p}\left(\mathbf{R}_{l}\right)\right\rangle$. Note that the coefficients of the hopping terms in Eq. (46) are the hopping amplitudes in Fig. 8, those along the bonds marked by dashed lines are given by Eq. (38), while those along the bonds marked by dotted lines are exactly zero. Since the hopping problems for $h=1$ holes break the translational symmetry of the lattice, it is necessary to divide each sublattice $A$ and $B$ into two further sublattices: $A=A^{\prime} \cup A^{\prime \prime}$ and $B=B^{\prime} \cup B^{\prime \prime}$, where sites in the sublattices $A^{\prime}$ and $B^{\prime}$ are pairwise connected by $z$ bonds in even stripes, and sites in the sublattices $A^{\prime \prime}$ and $B^{\prime \prime}$ are pairwise connected by $z$ bonds in odd stripes.

The Hamiltonian in Eq. (46) is quadratic, and therefore it becomes diagonal after an appropriate transformation of the singlehole operators $a_{h, q, p}^{(\dagger)}\left(\mathbf{R}_{l}\right)$. Due to the translational symmetry of the hopping problems, the new single-hole operators $\tilde{a}_{h, q, p}^{(\dagger)}(\mathbf{k}, \nu)$ are labeled with the lattice momentum $\mathbf{k}=\left(k_{X}, k_{Y}\right)$ conjugate to the lattice position $\mathbf{R}=(X, Y)$. In terms of the original real-space operators, these new momentum-space operators are given by

$$
\begin{aligned}
\tilde{a}_{0, q, p}(\mathbf{k}, \nu)= & \frac{1}{\sqrt{N}} \sum_{l \in A}\left[\beta_{0, q}^{A}(\mathbf{k}, \nu) a_{0, q, p}\left(\mathbf{R}_{l}\right) e^{-i \mathbf{k} \cdot \mathbf{R}_{l}}+\beta_{0, q}^{B}(\mathbf{k}, \nu) a_{0, q, p}\left(\mathbf{R}_{z(l)}\right) e^{-i \mathbf{k} \cdot \mathbf{R}_{z(l)}}\right] \\
\tilde{a}_{1, q, p}(\mathbf{k}, \nu)= & \sqrt{\frac{2}{N}} \sum_{l \in A^{\prime}}\left[\beta_{1, q}^{A^{\prime}}(\mathbf{k}, \nu) a_{1, q, p}\left(\mathbf{R}_{l}\right) e^{-i \mathbf{k} \cdot \mathbf{R}_{l}}+\beta_{1, q}^{B^{\prime}}(\mathbf{k}, \nu) a_{1, q, p}\left(\mathbf{R}_{z(l)}\right) e^{-i \mathbf{k} \cdot \mathbf{R}_{z(l)}}\right. \\
& \left.+\beta_{1, q}^{A^{\prime \prime}}(\mathbf{k}, \nu) a_{1, q, p}\left(\mathbf{R}_{x(z(l))}\right) e^{-i \mathbf{k} \cdot \mathbf{R}_{x(z(l))}}+\beta_{1, q}^{B^{\prime \prime}}(\mathbf{k}, \nu) a_{1, q, p}\left(\mathbf{R}_{y(l)}\right) e^{-i \mathbf{k} \cdot \mathbf{R}_{y(l)}}\right]
\end{aligned}
$$

where the coefficients $\beta_{h, q}(\mathbf{k}, \nu) \sim 1$ for the different sublattices distinguish two bands $\nu=\{1,2\}$ in the case of $h=0$ and four bands $\nu=\{1,2,3,4\}$ in the case of $h=1$. In terms of the momentum-space operators $\tilde{a}_{h, q, p}^{(\dagger)}(\mathbf{k}, \nu)$, the Hamiltonian in Eq. (46) takes the free-particle form

$$
H_{a}=\Gamma_{0}+\sum_{h, q, p} \sum_{\mathbf{k}, \nu} \Lambda_{h, q}(\mathbf{k}, \nu) \tilde{n}_{h, q, p}(\mathbf{k}, \nu)
$$

where $\tilde{n}_{h, q, p}(\mathbf{k}, \nu) \equiv \tilde{a}_{h, q, p}^{\dagger}(\mathbf{k}, \nu) \tilde{a}_{h, q, p}(\mathbf{k}, \nu)$ is the number operator in momentum space. The constraint on the total number of holes is then $n=\sum_{h, q, p} \sum_{\mathbf{k}, \nu}\left\langle\tilde{n}_{h, q, p}(\mathbf{k}, \nu)\right\rangle$.

To evaluate the multi-hole energy as the expectation value of the Hamiltonian in Eq. 48, we need to know the energies $\Lambda_{h, q}(\mathbf{k}, \nu)$ and the occupation numbers $\left\langle\tilde{n}_{h, q, p}(\mathbf{k}, \nu)\right\rangle$ of the single-hole states. In the ground state of the model, holes occupy only the lowest-energy single-hole states, and it is therefore enough to determine the single-hole energy $\Lambda_{h, q}(\mathbf{k}, \nu)$ around its overall minimum. On the other hand, the overall minimum of $\Lambda_{h, q}(\mathbf{k}, \nu)$ in the lowest band $\nu=1$ is at zero momentum because the hopping amplitudes in Fig. 8 are all non-positive. Expanding $\Lambda_{h, q}(\mathbf{k}, 1)$ up to quadratic order in the momentum around $\mathbf{k}=\mathbf{0}$, and keeping the lowest-order terms in $J \ll 1$, the single-hole dispersion relations for the different quantum numbers are given by

$$
\begin{aligned}
& \Lambda_{0,0}(\mathbf{k}, 1)=\left[-2+\frac{3 k_{X}^{2}}{8}+\frac{9 k_{Y}^{2}}{16}\right] t \\
& \Lambda_{0,1}(\mathbf{k}, 1)=\left[-1+\frac{3 k_{X}^{2}}{8}+\frac{27 J^{4} k_{Y}^{2}}{128}\right] t, \\
& \Lambda_{1, q}(\mathbf{k}, 1)=-\frac{9 J^{8}}{1024}+\left[-\frac{1+\sqrt{5}}{2}+\frac{3 k_{X}^{2}}{4 \sqrt{5}}\right] t .
\end{aligned}
$$

Since $h=1$ holes are not allowed to hop at all between their stripes, their dispersion relation is independent of the component $k_{Y}$ at all orders of the momentum.

When turning our attention to the corresponding occupation numbers $\left\langle\tilde{n}_{h, q, p}(\mathbf{k}, 1)\right\rangle$ around zero momentum, we assume that all holes in the multi-hole state have identical quantum numbers $h$ and $q$. It is then crucial to notice that certain holes are bosons, while others are fermions. If the holes are bosons, they all occupy the zero-momentum state. For holes with $h=0$ and $q=1$, the average single-hole energy in the multi-hole state is then $\left\langle\Lambda_{0,1}(\mathbf{k}, \nu)\right\rangle=-t$. If the holes are fermions, they fill up a Fermi sea around the zero-momentum state: each state inside the Fermi surface is occupied by two holes with different quantum numbers $p=\{0,1\}$, while the states outside the Fermi surface are unoccupied. For holes 


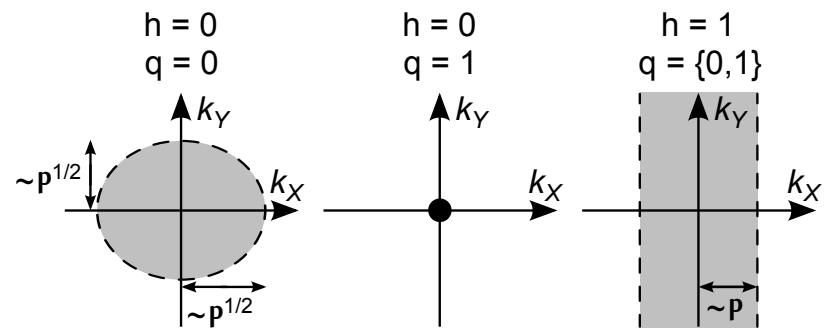

FIG. 12: Occupations of the single-hole states for different combinations of the flux quantum number $h=\{0,1\}$ and the fermion quantum number $q=\{0,1\}$. In the bosonic case, the lowest-energy state of macroscopic occupation is marked by a black dot. In the fermionic cases, the Fermi sea states of constant occupation are marked by gray shading, while the Fermi surface separating occupied and unoccupied states is marked by a dashed line.

with $h=0$ and $q=0$, the equipotential curves are ellipses of similar half-axes. The Fermi sea is therefore an ellipse of halfaxes $\Delta k_{X} \sim \Delta k_{Y} \sim \sqrt{\rho}$, and the average single-hole energy is $\left\langle\Lambda_{0,0}(\mathbf{k}, \nu)\right\rangle=-2 t+\kappa_{1} t \rho$, where $\kappa_{1} \sim 1$. For holes with $h=1$ and $q=\{0,1\}$, the equipotential curves are lines parallel to the $k_{Y}$ direction. The Fermi sea is therefore a strip of half-width $\Delta k_{X} \sim \rho$, and the average single-hole energy is $\left\langle\Lambda_{1, q}(\mathbf{k}, \nu)\right\rangle=-9 J^{8} / 1024-(1+\sqrt{5}) t / 2+\kappa_{2} t \rho^{2}$, where $\kappa_{2} \sim 1$. The occupation numbers of the single-hole states for the different quantum numbers $h$ and $q$ are illustrated in Fig.12, while the resulting average single-hole energies in the multi-hole state are summarized in Table VIII.

\begin{tabular}{c|c|c}
\hline \hline \multicolumn{2}{c|}{ Hole type } & Average single-hole energy \\
\hline \multirow{2}{*}{$h=0$} & $q=0$ & $-2 t+\kappa_{1} t \rho$ \\
& $q=1$ & $-t$ \\
\hline \multirow{2}{*}{$h=1$} & $q=0$ \\
& $q=1$ & $-9 J^{8} / 1024-(1+\sqrt{5}) t / 2+\kappa_{2} t \rho^{2}$ \\
\hline \hline
\end{tabular}

TABLE VIII: Average single-hole energy $\left\langle\Lambda_{h, q}(\mathbf{k}, \nu)\right\rangle$ in the model with a density $\rho \ll 1$ of mobile holes with flux quantum numbers $h=\{0,1\}$ and fermion quantum numbers $q=\{0,1\}$.

We are now ready to identify the ground-state quantum numbers of the model. Since the total number of holes is fixed, the average single-hole energies $\left\langle\Lambda_{h, q}(\mathbf{k}, \nu)\right\rangle$ for the different quantum numbers can be compared directly. Furthermore, the assumption of small hole density $\rho \ll 1$ means that the energies $\sim t \rho$ and $\sim t \rho^{2}$ are negligible compared to the energies $\sim t$. The results in TableVIII then indicate two complementary regimes in the behavior of the model. In the first regime with $J^{8} \ll t \ll J^{4}$, holes with $h=0$ and $q=0$ have the lowest average energy. This means that all holes in the ground state have quantum numbers $h=0$ and $q=0$. At each momentum $\mathbf{k}$ within the Fermi ellipse of Fig. 12, there are two holes with quantum numbers $p=\{0,1\}$. In the second regime with $t \ll J^{8}$, holes with $h=1$ and $q=\{0,1\}$ have the lowest average energy. This means that all holes in the ground state have quantum numbers $h=1$. At each momentum $\mathbf{k}$ within the Fermi strip of Fig. 12 there are four holes with quantum numbers $q=\{0,1\}$ and $p=\{0,1\}$. Note that our original assumption of no anyonic relative statistics is self-consistent as all holes in the ground state are $h=0$ holes in the first regime and $h=1$ holes in the second regime.

Due to the distinct ground states, the model also has different physical properties in the two regimes. We consider the net magnetization and the electrical conductivities in the $X$ and $Y$ directions. The net magnetization is the sum of the local hole magnetizations $(-1)^{q+p}$ and is zero in both regimes because each hole with quantum numbers $h, q$, and $p$ has a pair with quantum numbers $h, q$, and $1-p$. In terms of the partial densities $\rho_{h, q}=\sum_{p} \sum_{\mathbf{k}}\left\langle\tilde{n}_{h, q, p}(\mathbf{k}, 1)\right\rangle / 2 N$ of the various hole types, the conductivities in the two directions are

$$
\sigma_{X, Y}^{*}=e_{*}^{2} \tau \sum_{h, q} \rho_{h, q}\left[\frac{\partial^{2} \Lambda_{h, q}(\mathbf{k}, 1)}{\partial k_{X, Y}^{2}}\right]_{\mathbf{k}=\mathbf{0}},
$$

where $e_{*}$ is the hole charge, and $\tau$ is the elastic scattering time. In the first regime with $J^{8} \ll t \ll J^{4}$, the partial hole densities are $\rho_{0,0}=\rho$ and $\rho_{1, q}=\rho_{h, 1}=0$. Since the effective masses $\left[\partial^{2} \Lambda_{0,0}(\mathbf{k}, 1) / \partial k_{X, Y}^{2}\right]^{-1}$ are similar in the $X$ and $Y$ directions, the conductivity is approximately isotropic: $\sigma_{X}^{*} \sim \sigma_{Y}^{*} \sim t \rho e_{*}^{2} \tau$. In the second regime with $t \ll J^{8}$, the partial hole densities are $\rho_{1, q}=\rho / 2$ and $\rho_{0, q}=0$. Since the effective masses $\left[\partial^{2} \Lambda_{1, q}(\mathbf{k}, 1) / \partial k_{X, Y}^{2}\right]^{-1}$ are finite in the $X$ direction and infinite in the $Y$ direction, the conductivity is extremely anisotropic: $\sigma_{X}^{*} \sim t \rho e_{*}^{2} \tau$ and $\sigma_{Y}^{*}=0$.

\section{B. Mean-field treatment of interactions}

We now consider hole interactions in the model with a small density $\rho \ll 1$ of mobile holes. To represent an interaction of strength $\Delta \Gamma_{0}$ between two holes at a relative lattice position $\mathbf{R}=\mathbf{R}_{2}-\mathbf{R}_{1}$, we need to add an appropriate quartic term to the Hamiltonian in Eq. (46). Restricting our attention to the Coulomb repulsion and the two attraction mechanisms discussed in Sec.VC this quartic term takes the general form

$$
\begin{aligned}
\Delta H_{a}= & \Delta \Gamma_{0} \sum_{\mathbf{R}_{1}} a_{h_{1}, q_{1}^{\prime}, p_{1}}^{\dagger}\left(\mathbf{R}_{1}\right) a_{h_{2}, q_{2}^{\prime}, p_{2}}^{\dagger}\left(\mathbf{R}_{1}+\mathbf{R}\right) \\
& \times a_{h_{2}, q_{2}, p_{2}}\left(\mathbf{R}_{1}+\mathbf{R}\right) a_{h_{1}, q_{1}, p_{1}}\left(\mathbf{R}_{1}\right) .
\end{aligned}
$$

The flux quantum numbers $h_{1,2}$ and the plaquette quantum numbers $p_{1,2}$ are conserved by such a general hole interaction, while the fermion quantum numbers $q_{1,2} \neq q_{1,2}^{\prime}$ satisfy the relation $q_{1}^{\prime}+q_{2}^{\prime}=q_{1}+q_{2}$ modulo 2 so that the global constraint $\sum_{j} q_{j}=$ even is not violated.

Since the flux quantum numbers are conserved, the two complementary regimes found in Sec. VII A remain applicable in the presence of hole interactions: all holes in the ground state have quantum numbers $h=0$ in the first regime with $J^{8} \ll t \ll J^{4}$, while they all have quantum numbers $h=1$ in the second regime with $t \ll J^{8}$. We can then consider the two regimes independently from each other with only $h \equiv h_{1,2}=0$ holes in the first regime and only $h \equiv h_{1,2}=1$ holes in the second regime. On the other hand, this means that 
our assumption of no anyonic relative statistics remains selfconsistent in the presence of hole interactions. Expressing the real-space operators $a_{h, q, p}^{(\dagger)}\left(\mathbf{R}_{l}\right)$ in terms of the momentumspace operators $\tilde{a}_{h, q, p}^{(\dagger)}(\mathbf{k}, \nu)$, and considering only the lowest band $\nu=1$, the quartic term in Eq. (51) becomes

$$
\begin{aligned}
\Delta H_{a}= & \frac{\Delta \Gamma_{0}}{N} \sum_{\mathbf{k}_{1}, \mathbf{k}_{2}, \mathbf{k}^{\prime}} \Upsilon_{h, \hat{q}}(\mathbf{k}) e^{i \mathbf{k}^{\prime} \cdot \mathbf{R}} \tilde{a}_{h, q_{1}^{\prime}, p_{1}}^{\dagger}\left(\mathbf{k}_{1}+\mathbf{k}^{\prime}\right) \\
& \times \tilde{a}_{h, q_{2}^{\prime}, p_{2}}^{\dagger}\left(\mathbf{k}_{2}-\mathbf{k}^{\prime}\right) \tilde{a}_{h, q_{2}, p_{2}}\left(\mathbf{k}_{2}\right) \tilde{a}_{h, q_{1}, p_{1}}\left(\mathbf{k}_{1}\right)
\end{aligned}
$$

where $\tilde{a}_{h, q, p}^{(\dagger)}(\mathbf{k}) \equiv \tilde{a}_{h, q, p}^{(\dagger)}(\mathbf{k}, 1)$, and the quantity $\Upsilon_{h, \hat{q}}(\mathbf{k}) \sim 1$ with $\hat{q} \equiv\left\{q_{1}, q_{2}, q_{1}^{\prime}, q_{2}^{\prime}\right\}$ depends on the various coefficients $\beta_{h, q}(\mathbf{k}) \equiv \beta_{h, q}(\mathbf{k}, 1) \sim 1$ for the different sublattices.

The behavior of the model is influenced by hole interactions in several ways. We aim to specify the extent of applicability of the results in Sec.VII A for the ground-state quantum numbers and the corresponding physical properties. To this end, we investigate how hole interactions renormalize the average single-hole energies in Table VIII as a function of the partial hole densities $\rho_{h, q}$. In practice, we apply a standard meanfield decomposition to the Hamiltonian: each quartic term in Eq. 52) is decomposed into two constituent quadratic terms, and each quadratic term is coupled to the expectation value of the other one. The single-hole energies $\Lambda_{h, q}(\mathbf{k}) \equiv \Lambda_{h, q}(\mathbf{k}, 1)$ in Eq. (48) are then renormalized by the mean-field decomposition of any quartic term that is a product of two number operators $\tilde{n}_{h, q, p}(\mathbf{k}) \equiv \tilde{n}_{h, q, p}(\mathbf{k}, 1)$. In general, if we keep all such quartic terms in Eq. (52), and include all the equivalent quartic terms that differ only in their conserved plaquette quantum numbers $p_{1,2}$, the resulting mean-field decomposition takes the approximate form

$$
\begin{aligned}
\Delta \tilde{H}_{a} \sim & \frac{\Delta \Gamma_{0}}{N} \sum_{\mathbf{k}_{1}, \mathbf{k}_{2}}\left[\left\langle\tilde{n}_{h, q_{1}}\left(\mathbf{k}_{1}\right)\right\rangle \tilde{n}_{h, q_{2}}\left(\mathbf{k}_{2}\right)\right. \\
& \left.+\left\langle\tilde{n}_{h, q_{2}}\left(\mathbf{k}_{2}\right)\right\rangle \tilde{n}_{h, q_{1}}\left(\mathbf{k}_{1}\right)\right]
\end{aligned}
$$

where $\tilde{n}_{h, q}(\mathbf{k}) \equiv \sum_{p} \tilde{n}_{h, q, p}(\mathbf{k})$. Since $\rho=\sum_{h, q} \rho_{h, q}$ and $\sum_{\mathbf{k}}\left\langle\tilde{n}_{h, q}(\mathbf{k})\right\rangle=2 N \rho_{h, q}$, the single-hole energies in Eq. (48) are renormalized by $\Delta \Lambda_{h, q}(\mathbf{k}) \sim \Delta \Gamma_{0} \rho$.

\section{First regime: $J^{8} \ll t \ll J^{4}$}

In the first regime with $J^{8} \ll t \ll J^{4}$, all holes in the ground state have flux quantum numbers $h=0$, and therefore all quartic terms in Eq. 51) have $h \equiv h_{1,2}=0$. In the region around zero momentum occupied by holes, the coefficients $\beta_{0, q}(\mathbf{k})$ for the two sublattices $A$ and $B$ are

$$
\begin{array}{ll}
\beta_{0,0}^{A}(\mathbf{k})=\frac{1}{\sqrt{2}}, & \beta_{0,0}^{B}(\mathbf{k})=\frac{1}{\sqrt{2}} e^{i k_{Y} / 4} \\
\beta_{0,1}^{A}(\mathbf{k})=\frac{1}{\sqrt{2}}, & \beta_{0,1}^{B}(\mathbf{k})=\frac{1}{\sqrt{2}} e^{-i k_{Y} / 2} .
\end{array}
$$

Furthermore, the total hole density is $\rho=\rho_{0,0}+\rho_{0,1}$ in terms of the partial hole densities $\rho_{h, q}$. (a)

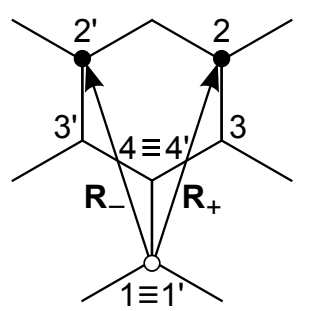

(b)

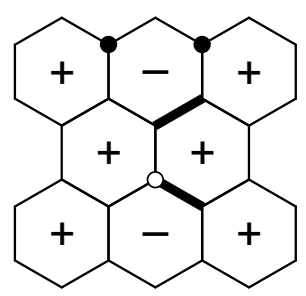

FIG. 13: (a) Site labeling convention around two interacting holes (white and black dots) at the relative lattice positions $\mathbf{R}_{ \pm}$. (b) Bond fermion sector around the same two holes when fluxes are bound to them: it is labeled with the excited bond fermions (thick lines) and the corresponding plaquette operator eigenvalues $( \pm 1)$.

It is instructive to first consider hole interactions that conserve the fermion quantum numbers $q_{1,2}=q_{1,2}^{\prime}$ and are also independent of them. Hole interactions of this type include the Coulomb repulsion and the first attraction mechanism of Sec. VC In this case, each quartic term in Eq. (52) with $\mathbf{k}^{\prime}=\mathbf{0}$ is a product of two number operators. Keeping only the terms with $\mathbf{k}^{\prime}=\mathbf{0}$, using that $\Upsilon_{0, \hat{q}}(\mathbf{k})=1 / 4$ for all such terms, and summing over the quantum numbers $q_{1,2}$ and $p_{1,2}$, the mean-field decomposition becomes

$$
\begin{aligned}
\Delta \tilde{H}_{a}= & \frac{\Delta \Gamma_{0}}{4 N} \sum_{q_{1}, q_{2}} \sum_{\mathbf{k}_{1}, \mathbf{k}_{2}}\left[\left\langle\tilde{n}_{0, q_{1}}\left(\mathbf{k}_{1}\right)\right\rangle \tilde{n}_{0, q_{2}}\left(\mathbf{k}_{2}\right)\right. \\
& \left.+\left\langle\tilde{n}_{0, q_{2}}\left(\mathbf{k}_{2}\right)\right\rangle \tilde{n}_{0, q_{1}}\left(\mathbf{k}_{1}\right)\right] .
\end{aligned}
$$

Since $\sum_{q} \sum_{\mathbf{k}}\left\langle\tilde{n}_{0, q}(\mathbf{k})\right\rangle=2 N \rho$ for both equivalent terms inside its square brackets, Eq. (55) reduces to

$$
\Delta \tilde{H}_{a}=\Delta \Gamma_{0} \rho \sum_{q} \sum_{\mathbf{k}} \tilde{n}_{0, q}(\mathbf{k}) .
$$

From a comparison between Eqs. (48) and (56), we conclude that the single-hole energies for $h=0$ and $q=\{0,1\}$ are renormalized by $\Delta \Lambda_{0, q}(\mathbf{k})=\Delta \Gamma_{0} \rho$. Since this energy depends only on the total hole density $\rho$, it corresponds to a constant shift for all the single-hole energies. This means that the results for the ground state in Sec. VIIA are not affected by hole interactions of this type.

Importantly, the second attraction mechanism of Sec. VC switches the fermion quantum numbers $q_{1,2}=1-q_{1,2}^{\prime}$. It is therefore represented by quartic terms in Eq. (52) where either $q_{1}=q_{2}$ and $q_{1}^{\prime}=q_{2}^{\prime}$ or $q_{1}=q_{2}^{\prime}$ and $q_{1}^{\prime}=q_{2}$. In the first case, the quartic term is never a product of two number operators, while in the second case, it is a product of two number operators when $\mathbf{k}^{\prime}=\mathbf{k}_{2}-\mathbf{k}_{1}$. According to the discussion in Sec. VC this interaction has the largest strength $\left|\Delta \Gamma_{0}\right|=1$ when the two holes are at neighboring sites connected by a $z$ bond, or equivalently, at a relative lattice position $\mathbf{R}_{z}=(0,1)$. However, the two holes in this case have a mutual hole fermion, and therefore their fermion quantum numbers become ill-defined. If we require the fermion quantum numbers to be well-defined, the interaction has the largest 
strength $\left|\Delta \Gamma_{0}\right|=J$ when the two holes are at the relative lattice positions $\mathbf{R}_{ \pm}=( \pm \sqrt{3} / 2,5 / 2)$ shown in Fig. [13, Setting $q_{1}=q_{2}^{\prime}=\tilde{q}$ and $q_{1}^{\prime}=q_{2}=1-\tilde{q}$, keeping only the terms with $\mathbf{k}^{\prime}=\mathbf{k}_{2}-\mathbf{k}_{1}$ for both relative positions $\mathbf{R}_{ \pm}$, and summing over $\tilde{q}$ and $p_{1,2}$, the mean-field decomposition becomes

$$
\begin{aligned}
& \Delta \tilde{H}_{a}= \frac{1}{N} \sum_{\tilde{q}} \sum_{\mathbf{k}_{1}, \mathbf{k}_{2}} \sum_{ \pm} \Delta \Gamma_{0}(\tilde{q}, \pm) \Upsilon_{0, \hat{q}}(\mathbf{k}) e^{i\left(\mathbf{k}_{2}-\mathbf{k}_{1}\right) \cdot \mathbf{R}_{ \pm}} \\
& \times\left[\left\langle\tilde{n}_{0, \tilde{q}}\left(\mathbf{k}_{1}\right)\right\rangle \tilde{n}_{0,1-\tilde{q}}\left(\mathbf{k}_{2}\right)+\left\langle\tilde{n}_{0,1-\tilde{q}}\left(\mathbf{k}_{2}\right)\right\rangle \tilde{n}_{0, \tilde{q}}\left(\mathbf{k}_{1}\right)\right] \\
& \Upsilon_{0, \hat{q}}(\mathbf{k})= \begin{cases}\frac{1}{4} e^{i\left(\mathbf{k}_{1}+2 \mathbf{k}_{2}\right) \cdot \mathbf{R}_{z} / 4} & (\tilde{q}=0) \\
\frac{1}{4} e^{-i\left(2 \mathbf{k}_{1}+\mathbf{k}_{2}\right) \cdot \mathbf{R}_{z} / 4} & (\tilde{q}=1)\end{cases}
\end{aligned}
$$

The four interaction strengths $\Delta \Gamma_{0}(\tilde{q}, \pm)$ can be obtained by treating the Ising interactions $-J \sigma_{l}^{x} \sigma_{x(l)}^{x}$ and $-J \sigma_{l}^{y} \sigma_{y(l)}^{y}$ as perturbations around the isolated dimer limit. Using the site labeling convention in Fig. 13, the interaction strength in the case of $\tilde{q}=0$ for the relative position $\mathbf{R}_{+}$is

$$
\begin{aligned}
\Delta \Gamma_{0}(0,+) & =+J\left\langle 0\left|f_{1}\left(i b_{3}^{x} c_{3}\right)\left(i b_{4}^{x} c_{4}\right) f_{3}^{\dagger}\right| 0\right\rangle \\
& =-i J\left\langle 0\left|\hat{u}_{3,4} f_{1} c_{3} c_{4} f_{3}^{\dagger}\right| 0\right\rangle=-J u_{3,4}=-J,
\end{aligned}
$$

where an additional minus sign arises because the corresponding quartic term in Eq. (51) does not only transfer the bound matter fermion from $\mathbf{R}_{2}$ to $\mathbf{R}_{1}$ but also exchanges the two bare holes at $\mathbf{R}_{1,2}$. Note that $u_{l, \alpha(l)}=+1$ for all bonds because no bond fermions are excited. The interaction strength in the other case $\tilde{q}=1$ is the Hermitian conjugate of Eq. [58, while that for the other relative position $\mathbf{R}_{-}$is equivalent to it via site relabeling, and thus $\Delta \Gamma_{0}(\tilde{q}, \pm)=-J$ in both cases and for both relative positions. On the other hand, this implies that the mean-field decomposition in Eq. (57) becomes

$$
\begin{aligned}
\Delta \tilde{H}_{a}= & -\frac{J}{N} \sum_{\mathbf{k}_{1}, \mathbf{k}_{2}} \cos \left[\frac{\sqrt{3}}{2} \tilde{k}_{X}\right] \cos \left[\frac{9}{4} k_{1, Y}-3 k_{2, Y}\right] \\
& \times\left[\left\langle\tilde{n}_{0,0}\left(\mathbf{k}_{1}\right)\right\rangle \tilde{n}_{0,1}\left(\mathbf{k}_{2}\right)+\left\langle\tilde{n}_{0,1}\left(\mathbf{k}_{2}\right)\right\rangle \tilde{n}_{0,0}\left(\mathbf{k}_{1}\right)\right],
\end{aligned}
$$

where $\tilde{\mathbf{k}}=\left(\tilde{k}_{X}, \tilde{k}_{Y}\right) \equiv \mathbf{k}_{1}-\mathbf{k}_{2}$ is the relative lattice momentum. Since the original single-hole energies $\Lambda_{0, q}(\mathbf{k})$ in Eq. (49) and their renormalizations $\Delta \Lambda_{0, q}(\mathbf{k})$ resulting from Eq. (59) are both minimal for $\mathbf{k}_{1,2}=\mathbf{0}$, the smallest renormalized single-hole energies $\Lambda_{0, q}^{\prime}(\mathbf{k}) \equiv \Lambda_{0, q}(\mathbf{k})+\Delta \Lambda_{0, q}(\mathbf{k})$ are obtained if holes occupy the single-hole states around zero momentum. By approximating $\sum_{\mathbf{k}} \psi(\mathbf{k})\left\langle\tilde{n}_{0, q}(\mathbf{k})\right\rangle$ with $\psi(\langle\mathbf{k}\rangle) \sum_{\mathbf{k}}\left\langle\tilde{n}_{0, q}(\mathbf{k})\right\rangle$ for any function $\psi(\mathbf{k})$ in terms of the respective central momenta $\left\langle\mathbf{k}_{1,2}\right\rangle=\mathbf{0}$, and making use of $\sum_{\mathbf{k}}\left\langle\tilde{n}_{0, q}(\mathbf{k})\right\rangle=2 N \rho_{0, q}$, Eq. (59) reduces to

$$
\begin{aligned}
\Delta \tilde{H}_{a}= & -2 J \rho_{0,1} \sum_{\mathbf{k}} \cos \left[\frac{\sqrt{3}}{2} k_{X}\right] \cos \left[\frac{9}{4} k_{Y}\right] \tilde{n}_{0,0}(\mathbf{k}) \\
& -2 J \rho_{0,0} \sum_{\mathbf{k}} \cos \left[\frac{\sqrt{3}}{2} k_{X}\right] \cos \left[3 k_{Y}\right] \tilde{n}_{0,1}(\mathbf{k}) .
\end{aligned}
$$

From a comparison between Eqs. (48) and (60), we conclude that the single-hole energies for $h=0$ and $q=\{0,1\}$ are approximately renormalized by $\Delta \Lambda_{0, q}(\mathbf{0})=-2 J \rho_{0,1-q}$ in the region around zero momentum. If we keep only the leadingorder terms in $\rho \ll 1$, the average single-hole energies in Table VIII are then given by $\left\langle\Lambda_{0,0}^{\prime}(\mathbf{k})\right\rangle=-2 t-2 J \rho_{0,1}$ and $\left\langle\Lambda_{0,1}^{\prime}(\mathbf{k})\right\rangle=-t-2 J \rho_{0,0}$.

Assuming that the results for the ground state in Sec.VIIA remain applicable so that $\rho_{0,0}=\rho$ and $\rho_{0,1}=0$, the two average single-hole energies $\left\langle\Lambda_{0, q}^{\prime}(\mathbf{k})\right\rangle$ become equal at the critical hole density $\rho=\rho_{C}=t / 2 J$. At subcritical densities $\rho<\rho_{C}$, we find that $\left\langle\Lambda_{0,0}^{\prime}(\mathbf{k})\right\rangle<\left\langle\Lambda_{0,1}^{\prime}(\mathbf{k})\right\rangle$ for all possible values of the partial densities $\rho_{0, q}$. This means that the ground-state values are $\rho_{0,0}=\rho$ and $\rho_{0,1}=0$, and that the results in Sec. VIIA indeed remain applicable. At supercritical densities $\rho>\rho_{C}$, there are equilibrium values of the partial densities $\rho_{0, q}$ at which $\left\langle\Lambda_{0,0}^{\prime}(\mathbf{k})\right\rangle=\left\langle\Lambda_{0,1}^{\prime}(\mathbf{k})\right\rangle$. By solving $\left\langle\Lambda_{0,0}^{\prime}(\mathbf{k})\right\rangle=\left\langle\Lambda_{0,1}^{\prime}(\mathbf{k})\right\rangle$ and $\rho=\rho_{0,0}+\rho_{0,1}$ for the two unknowns $\rho_{0, q}$, the ground-state values are

$$
\rho_{0,0}=\frac{1}{2}\left(\rho+\rho_{C}\right), \quad \rho_{0,1}=\frac{1}{2}\left(\rho-\rho_{C}\right) .
$$

To summarize, only holes with $h=0$ and $q=0$ are present in the low-density limit $\rho \rightarrow 0$, while holes with $h=0$ and $q=1$ appear above the critical density $\rho=\rho_{C}$. Note that $\rho_{C}=t / 2 J$ is small due to $t \ll J^{4}$.

The subcritical and the supercritical regimes are also distinct in terms of their physical properties. At subcritical densities, the physical properties of the model are as discussed in Sec. VIIA except for a renormalization of the effective masses and hence the electrical conductivities. At supercritical densities, the physical properties are changed in an essential way by the presence of holes with $h=0$ and $q=1$. Since these holes are bosons, they all condense into the lowestenergy single-hole state at zero momentum. This condensation then leads to charged superfluid behavior in the presence of the Coulomb repulsion. Furthermore, due to the coherent condensation of both $p=0$ holes and $p=1$ holes, the model spontaneously develops a net magnetization.

\section{Second regime: $t \ll J^{8}$}

In the second regime with $t \ll J^{8}$, all holes in the ground state have flux quantum numbers $h=1$, and therefore all quartic terms in Eq. (51) have $h \equiv h_{1,2}=1$. The coefficients $\beta_{1, q}(\mathbf{k})$ for the four sublattices $A^{\prime}, B^{\prime}, A^{\prime \prime}$, and $B^{\prime \prime}$ are

$$
\begin{aligned}
& \beta_{1,0}^{A^{\prime}}(\mathbf{k})=\beta_{1,1}^{A^{\prime \prime}}(\mathbf{k})=\xi_{1}\left(k_{X}\right), \\
& \beta_{1,0}^{B^{\prime}}(\mathbf{k})=\beta_{1,1}^{B^{\prime \prime}}(\mathbf{k})=\xi_{1}\left(k_{X}\right) e^{i k_{Y}}, \\
& \beta_{1,0}^{A^{\prime \prime}}(\mathbf{k})=\beta_{1,1}^{A^{\prime}}(\mathbf{k})=\xi_{2}\left(k_{X}\right) e^{3 i k_{Y} / 2}, \\
& \beta_{1,0}^{B^{\prime \prime}}(\mathbf{k})=\beta_{1,1}^{B^{\prime}}(\mathbf{k})=\xi_{2}\left(k_{X}\right) e^{-i k_{Y} / 2},
\end{aligned}
$$

where $\xi_{1,2}\left(k_{X}\right) \in \mathbb{R}$, and $\xi_{1}^{2}\left(k_{X}\right)+\xi_{2}^{2}\left(k_{X}\right)=1 / 2$. Furthermore, the total hole density is $\rho=\rho_{1,0}+\rho_{1,1}$ in terms of the partial hole densities $\rho_{h, q}$. 

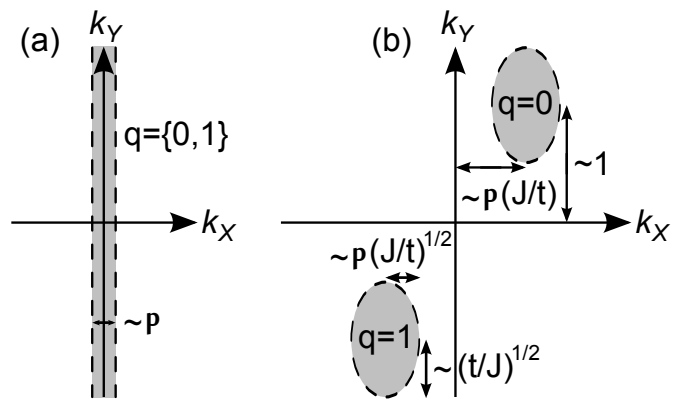

FIG. 14: Single-hole states occupied by holes with quantum numbers $h=1$ and $q=\{0,1\}$ in the non-interacting treatment (a) and in the interacting treatment (b). Fermi sea states of constant occupation are marked by gray shading, while the Fermi surface separating occupied and unoccupied states is marked by a dashed line.

We first notice that the mean-field decomposition of a hole interaction that is independent of the conserved fermion quantum numbers $q_{1,2}$ no longer takes the form of Eq. (56). Since $\Upsilon_{1, \hat{q}}(\mathbf{k})$ is not $1 / 4$ for all quartic terms with $\mathbf{k}^{\prime}=\mathbf{0}$ in Eq. (52), the renormalizations $\Delta \Lambda_{1, q}(\mathbf{k})$ of the single-hole energies become dependent on the individual partial hole densities $\rho_{1, q}$. If we consider the Coulomb repulsion and the first attraction mechanism of Sec. $\mathrm{VC}$ for all relative lattice positions $\mathbf{R}$, the single-hole energies in Eq. (48) are renormalized by $\Delta \Lambda_{1, q}(\mathbf{k})=\Delta \Gamma_{0}^{\prime} \rho_{1, q}+\Delta \Gamma_{0}^{\prime \prime} \rho_{1,1-q}$, where the exact values of $\Delta \Gamma_{0}^{\prime}$ and $\Delta \Gamma_{0}^{\prime \prime}$ depend on the detailed form of the Coulomb repulsion. In the case of $\Delta \Gamma_{0}^{\prime}>\Delta \Gamma_{0}^{\prime \prime}$, the partial hole densities remain $\rho_{1,0}=\rho_{1,1}=\rho / 2$, while in the case of $\Delta \Gamma_{0}^{\prime}<\Delta \Gamma_{0}^{\prime \prime}$, the partial hole densities become either $\rho_{1,0}=\rho$ and $\rho_{1,1}=0$ or $\rho_{1,0}=0$ and $\rho_{1,1}=\rho$. We assume the first case in the following so that there are equal densities of $q=0$ holes and $q=1$ holes in the ground state.

For the second attraction mechanism of Sec.VClat the relative lattice positions $\mathbf{R}_{ \pm}$, the mean-field decomposition takes the form of Eq. (57) with $\Upsilon_{1, \hat{q}}(\mathbf{k})=\frac{1}{4} \Xi\left(k_{X}\right) e^{i\left(\mathbf{k}_{2}-\mathbf{k}_{1}\right) \cdot \mathbf{R}_{z} / 2}$ and $\Xi\left(k_{X}\right)=16 \xi_{1}\left(k_{1, X}\right) \xi_{2}\left(k_{1, X}\right) \xi_{1}\left(k_{2, X}\right) \xi_{2}\left(k_{2, X}\right)$. Since Fig. 13 shows that $u_{3,4}=-1$ and $u_{3^{\prime}, 4^{\prime}}=+1$, the interaction strengths are $\Delta \Gamma_{0}(\tilde{q}, \pm)= \pm J$. On the other hand, this implies that mean-field decomposition becomes

$$
\begin{aligned}
\Delta \tilde{H}_{a}= & -\frac{J}{N} \sum_{\mathbf{k}_{1}, \mathbf{k}_{2}} \Xi\left(k_{X}\right) \sin \left[\frac{\sqrt{3}}{2} \tilde{k}_{X}\right] \sin \left[3 \tilde{k}_{Y}\right] \\
& \times\left[\left\langle\tilde{n}_{1,0}\left(\mathbf{k}_{1}\right)\right\rangle \tilde{n}_{1,1}\left(\mathbf{k}_{2}\right)+\left\langle\tilde{n}_{1,1}\left(\mathbf{k}_{2}\right)\right\rangle \tilde{n}_{1,0}\left(\mathbf{k}_{1}\right)\right]
\end{aligned}
$$

Unlike the original single-hole energies $\Lambda_{1, q}(\mathbf{k})$ in Eq. (49), their renormalizations $\Delta \Lambda_{1, q}(\mathbf{k})$ resulting from Eq. 63) are not minimal for $\mathbf{k}_{1}=\mathbf{k}_{2}=\mathbf{0}$. We therefore need to determine the ground-state occupations of the single-hole states that correspond to the smallest renormalized single-hole energies $\Lambda_{1, q}^{\prime}(\mathbf{k}) \equiv \Lambda_{1, q}(\mathbf{k})+\Delta \Lambda_{1, q}(\mathbf{k})$. Exploiting the equivalence between $q=0$ holes at momenta $\mathbf{k}_{1}$ and $q=1$ holes at momenta $\mathbf{k}_{2}$, and noticing that both sine factors in Eq. (63) depend only on the relative momentum $\tilde{\mathbf{k}} \equiv \mathbf{k}_{1}-\mathbf{k}_{\mathbf{2}}$, we conclude that the respective central momenta are related by $\mathbf{K}=\left(K_{X}, K_{Y}\right) \equiv\left\langle\mathbf{k}_{1}\right\rangle=-\left\langle\mathbf{k}_{2}\right\rangle$, and minimize the single-hole energies with respect to $\mathbf{K}$. Since $\Lambda_{1, q}(\mathbf{k})$ does not depend on the momentum component $k_{Y}$, the second sine factor in Eq. 63. can be maximized independently. In particular, its maximum $\sin \left[3\left\langle\tilde{k}_{Y}\right\rangle\right]=+1$ corresponds to the ground-state value $K_{Y}=\pi / 12$. Furthermore, if we assume $K_{X} \ll 1$, the first sine factor is approximately $\sqrt{3} K_{X}$. Due to $\sum_{\mathbf{k}}\left\langle\tilde{n}_{1, q}(\mathbf{k})\right\rangle=2 N \rho_{1, q}=N \rho$, the single-hole energies around the central momenta $\left\langle\mathbf{k}_{1,2}\right\rangle= \pm \mathbf{K}$ are then renormalized by $\Delta \Lambda_{1, q}(\mathbf{k}) \sim-J \rho K_{X}$, and the average single-hole energies in Table VIII take the form

$$
\left\langle\Lambda_{1, q}^{\prime}(\mathbf{k})\right\rangle=C^{\prime}(t, J)-\kappa_{0} J \rho K_{X}+\frac{3}{4 \sqrt{5}} t K_{X}^{2},
$$

where $C^{\prime}(t, J)$ and $\kappa_{0} \sim 1$ are independent of $K_{X}$. The minimum of $\left\langle\Lambda_{1, q}^{\prime}(\mathbf{k})\right\rangle$ with respect to $K_{X}$ corresponds to the ground-state value $K_{X} \sim J \rho / t$. By approximating $\sum_{\mathbf{k}} \psi(\mathbf{k})\left\langle\tilde{n}_{1, q}(\mathbf{k})\right\rangle$ with $\psi(\langle\mathbf{k}\rangle) \sum_{\mathbf{k}}\left\langle\tilde{n}_{1, q}(\mathbf{k})\right\rangle$ for any function $\psi(\mathbf{k})$ in terms of the central momenta $\left\langle\mathbf{k}_{1,2}\right\rangle= \pm \mathbf{K}$, and assuming $K_{X} \sim J \rho / t \ll 1$, Eq. (63) reduces to

$$
\begin{aligned}
\Delta \tilde{H}_{a}= & -\frac{J^{2} \rho^{2}}{t} \sum_{q} \sum_{\mathbf{k}} \tilde{\Xi}\left[k_{X}-(-1)^{q} K_{X}\right] \\
& \times \cos \left\{3\left[k_{Y}-(-1)^{q} K_{Y}\right]\right\} \tilde{n}_{1, q}(\mathbf{k}),
\end{aligned}
$$

where $\tilde{\Xi}\left[k_{X}-(-1)^{q} K_{X}\right] \sim 1$ contains all dependence on the momentum component $k_{X}$. Importantly, the renormalized single-hole energies $\Lambda_{1, q}^{\prime}(\mathbf{k})$ resulting from Eq. 65) depend on the momentum component $k_{Y}$ as well. In fact, the single-hole dispersion relations for $q=\{0,1\}$ holes around their respective central momenta $\pm \mathbf{K}$ are quadratic in both the $k_{X}$ and the $k_{Y}$ directions: the leading-order terms are $\sim t\left(k_{X} \mp K_{X}\right)^{2}$ and $\sim J^{2} \rho^{2}\left(k_{Y} \mp K_{Y}\right)^{2} / t$. This implies that the Fermi seas for the two hole types are ellipses of half-axes $\Delta k_{X} \sim \rho \sqrt{J / t}$ and $\Delta k_{Y} \sim \sqrt{t / J}$ centered at $\pm \mathbf{K}$. Note that $\Delta k_{Y} \ll 1$ due to $t \ll J^{8}$ and that $\Delta k_{X} \sim K_{X} \Delta k_{Y} \ll 1$ due to $K_{X} \ll 1$ and $\Delta k_{Y} \ll 1$. Since our calculation resulting in these Fermi ellipses is valid for any hole density $\rho>0$, the Fermi strip described in Sec. VII A is unstable against an arbitrarily small hole interaction. The Fermi ellipses of the interacting treatment and the Fermi strip of the non-interacting treatment are contrasted in Fig. 14

In terms of physical properties, the main difference with respect to the results in Sec. VIIA is a finite electrical conductivity in the $Y$ direction. The conductivities in the $X$ and $Y$ directions are still calculated by Eq. (50), except that we use the renormalized single-hole energies $\Lambda_{1, q}^{\prime}(\mathbf{k})$ and take their second derivatives at the central momenta $\pm \mathbf{K}$. Since the partial hole densities are $\rho_{1, q}=\rho / 2$, and the second derivatives are $\partial^{2} \Lambda_{1, q}^{\prime}(\mathbf{k}) / \partial k_{X}^{2} \sim t$ and $\partial^{2} \Lambda_{1, q}^{\prime}(\mathbf{k}) / \partial k_{Y}^{2} \sim J^{2} \rho^{2} / t$, the conductivities in the two directions become

$$
\sigma_{X}^{*} \sim t \rho e_{*}^{2} \tau, \quad \sigma_{Y}^{*} \sim \frac{J^{2} \rho^{3} e_{*}^{2} \tau}{t} .
$$

Note in particular that $\sigma_{X}^{*} \propto \rho$ and $\sigma_{Y}^{*} \propto \rho^{3}$. Since the ratio of the two conductivities is $\sigma_{Y}^{*} / \sigma_{X}^{*} \sim(J \rho / t)^{2} \ll 1$, the model has a strong conductivity anisotropy that becomes weaker as we increase the hole density $\rho$. 


\section{MOBILE HOLES BEYOND SLOW HOPPING}

By relaxing the condition of slow hopping, we qualitatively describe the Kitaev honeycomb model with mobile holes in the regimes of intermediate hopping $\left(J^{4} \ll t \ll 1\right)$ and fast hopping $(t \gg 1)$. We first consider a single isolated hole and investigate the applicability of the internal quantum numbers $h, q$, and $p$. Since the original definitions of these quantum numbers in Sec. $\mathrm{VB}$ are in terms of the internal modes only, they are not applicable beyond the limit of slow hopping when the excitations in the bulk modes can no longer be neglected. In the regime of intermediate hopping when $t \gg E_{P} \sim J^{4}$, the bulk flux excitations are no longer negligible, and the hole is surrounded by a cloud of fluctuating fluxes. In the regime of fast hopping when $t \gg E_{f} \sim 1$, the bulk fermion excitations are no longer negligible, and the hole is also surrounded by a cloud of fluctuating fermions. On the other hand, the hole combined with these excitation clouds has a well-defined superselection sector that is conserved by the hopping process due to the locality of the exchange operator $\mathcal{E}_{l, l^{\prime}}$. This means that the definitions of the quantum numbers $h$ and $q$ can be generalized in terms of their correspondence to these conserved superselection sectors. Furthermore, the only nontrivial terms in the exchange operator $\mathcal{E}_{l, l^{\prime}}$ are the Heisenberg terms in Eq. 23) that conserve the product of dimer operators $\lambda_{l}=\sigma_{l}^{z} \sigma_{z(l)}^{z}$ and the products of plaquette operators $W_{P}$ both in even stripes and in odd stripes (see Sec. VD). This means that the definitions of all quantum numbers $h, q$, and $p$ can be generalized in terms of these products such that they are conserved by the hopping process: $(-1)^{q+p}=\prod_{l \in A} \lambda_{l}$, $(-1)^{h+p}=\prod_{P \in \eta} W_{P}$, and $(-1)^{p}=\prod_{P \in \mu} W_{P}$, where each product is taken over a sufficiently large region that contains the clouds of fluctuating fluxes and fermions. The quantum numbers $h, q$, and $p$ are then valid if the distances between holes exceed the radii of these excitation clouds.

To provide an upper bound on the radius of each excitation cloud, we notice that the fluctuating fluxes and fermions increase the potential energy and decrease the kinetic energy of the hole. This means that the two radii are determined by a balance between the potential and the kinetic energies. Since the excitation energy of a bulk flux is $E_{P} \sim J^{4}$ and that of a bulk fermion is $E_{f} \sim 1$, the increase in the potential energy is on the order of $R_{P}^{2} E_{P} \sim R_{P}^{2} J^{4}$ for a flux cloud of radius $R_{P}$ and on the order of $R_{f}^{2} E_{f} \sim R_{f}^{2}$ for a fermion cloud of radius $R_{f}$. On the other hand, since the decrease in the kinetic energy due to both excitation clouds is at most $\sim t$, the increase in the potential energy due to either excitation cloud must be bounded by $\lesssim t$. We therefore conclude that the upper bound on the flux cloud radius is $R_{P} \lesssim \sqrt{t / J^{4}}$ and that on the fermion cloud radius is $R_{f} \lesssim \sqrt{t}$.

We are now ready to investigate the ground-state quantum numbers $h$ and $q$ for a finite density of mobile holes. For simplicity, we consider the case of $J^{4} \ll t \ll 1$ when only the plauqettes are fluctuating around the holes. To ensure that the quantum numbers $h$ and $q$ are valid, we assume a small hole density $\rho \ll R_{P}^{-2}$ and neglect any hole interactions. Since the hopping matrix elements are independent of the quantum numbers $p$, we also set $p=q$ for each hole without loss of generality. Due to the lack of broken dimers in the isolated dimer limit, the plaquette sector is then conserved by all hopping processes along $z$ bonds. Since the hopping processes along $x$ and $y$ bonds either flip no plaquettes or two plaquettes in each of two neighboring stripes (see Fig. 6), this implies that the number of excited plaquettes has a conserved parity in each stripe. If there are an odd number of excited plaquettes in any stripe around any hole, the given hole can hop in the $Y$ direction only if it leaves behind an excited plaquette in the given stripe. Since the kinetic energy decreases by $\sim t$ for each hole that can hop in the $Y$ direction, this process happens spontaneously for $t \gg J^{4}$, and there remain an even number of excited plaquettes in each stripe around each hole. Due to the relations $\prod_{P \in \eta} W_{P}=(-1)^{h+q}$ and $\prod_{P \in \mu} W_{P}=(-1)^{q}$, this means that any hole with quantum numbers other than $h=0$ and $q=0$ is unstable against a spontaneous decay into a hole with quantum numbers $h=0$ and $q=0$. Note that this result remains valid away from the isolated dimer limit and in the case of $t \gg 1$ when the fermions are also fluctuating. Holes with quantum numbers $h=0$ and $q=0$ are then energetically favorable because their hopping is the least constrained in the $Y$ direction.

In conclusion, the quantum numbers $h, q$, and $p$ generalize beyond the regime of slow hopping, but they are valid only for smaller hole densities due to the clouds of fluctuating excitations around holes. Furthermore, any hole with quantum numbers other than $h=0$ and $q=0$ is unstable against a spontaneous decay into a hole with quantum numbers $h=0$ and $q=0$. This means that all holes in the ground state have quantum numbers $h=0$ and $q=0$, and that the ground state is identical to that in the case of $J^{8} \ll t \ll J^{4}$. The only difference is that there are clouds of fluctuating excitations around each hole. Importantly, when the hole density becomes $\rho \gtrsim R_{P, f}^{-2}$, the clouds of fluctuating fluxes (fermions) around different holes merge, and the holes hop in an entire lattice of fluctuating fluxes (fermions).

\section{COMPARISON WITH MEAN-FIELD RESULTS}

\section{A. Holes in the parton description}

We now discuss the relation between our exact results for the Kitaev honeycomb model with mobile holes and the corresponding mean-field results in Ref. 23. In their description, the physical operators $\mathbf{c}_{l, \uparrow}^{(\dagger)}$ and $\mathbf{c}_{l, \downarrow}^{(\dagger)}$ that annihilate (create) a spin-up and a spin-down particle at site $l$, respectively, are expressed in terms of the fermionic spinon operators $\mathbf{f}_{l, \uparrow}^{(\dagger)}$ and $\mathbf{f}_{l, \downarrow}^{(\dagger)}$ and the bosonic holon operators $\mathbf{b}_{l, 1}^{(\dagger)}$ and $\mathbf{b}_{l, 2}^{(\dagger)}$. The resulting relations between the physical operators and the parton (holon and spinon) operators can be summarized in the matrix form $\mathbf{C}_{l}=\mathbf{F}_{l} \cdot \mathbf{B}_{l} / \sqrt{2}$, where the physical-operator matrix is

$$
\mathbf{C}_{l}=\left(\begin{array}{cc}
\mathbf{c}_{l, \uparrow} & -\mathbf{c}_{l, \downarrow}^{\dagger} \\
\mathbf{c}_{l, \downarrow} & \mathbf{c}_{l, \uparrow}^{\dagger}
\end{array}\right)
$$


while the spinon-operator and the holon-operator matrices are

$$
\mathbf{F}_{l}=\left(\begin{array}{cc}
\mathbf{f}_{l, \uparrow} & -\mathbf{f}_{l, \downarrow}^{\dagger} \\
\mathbf{f}_{l, \downarrow} & \mathbf{f}_{l, \uparrow}^{\dagger}
\end{array}\right), \quad \mathbf{B}_{l}=\left(\begin{array}{cc}
\mathbf{b}_{l, 1}^{\dagger} & -\mathbf{b}_{l, 2} \\
\mathbf{b}_{l, 2}^{\dagger} & \mathbf{b}_{l, 1}
\end{array}\right) .
$$

Importantly, the physical-operator matrix $\mathbf{C}_{l}$ is invariant under the combined gauge transformation $\mathbf{F}_{l} \rightarrow \mathbf{F}_{l} \cdot \mathbf{G}_{l}$ and $\mathbf{B}_{l} \rightarrow \mathbf{G}_{l} \cdot \mathbf{B}_{l}$ for any $\mathbf{G}_{l} \in \mathrm{SU}(2)$. Since a physical state should also be invariant under such an $\mathrm{SU}(2)$ gauge transformation at any site $l$, it must satisfy $\mathcal{K}_{l}^{\alpha}=0$ for all $\mathrm{SU}(2)$ generators $\mathcal{K}_{l}^{\alpha}$ with $\alpha=\{x, y, z\}$. If the spinon operators are related to the Majorana fermions introduced in Sec. IIIC by $\mathbf{f}_{l, \uparrow}=\left(c_{l}+i b_{l}^{z}\right) / 2$ and $\mathbf{f}_{l, \downarrow}=\left(i b_{l}^{x}-b_{l}^{y}\right) / 2$, these $\mathrm{SU}(2)$ generators take the form [see Eq. (20) in Ref. 23]

$\mathcal{K}_{l}^{\alpha}=\frac{i}{4} b_{l}^{\alpha} c_{l}-\frac{i}{8} \sum_{\alpha_{1}, \alpha_{2}} \tilde{\epsilon}_{\alpha \alpha_{1} \alpha_{2}} b_{l}^{\alpha_{1}} b_{l}^{\alpha_{2}}-\frac{1}{2} \sum_{\zeta_{1}, \zeta_{2}} \mathbf{b}_{l, \zeta_{1}} \tilde{\sigma}_{\zeta_{1} \zeta_{2}}^{\alpha} \mathbf{b}_{l, \zeta_{2}}^{\dagger}$,

where $\tilde{\sigma}^{\alpha}$ are the Pauli matrices, $\tilde{\epsilon}$ is the completely antisymmetric tensor, and the summations are over $\alpha_{1,2}=\{x, y, z\}$ and $\zeta_{1,2}=\{1,2\}$. For a single site $l$, there are only three physical states: the empty hole state $\left|\times_{l}\right\rangle$, the spin-up particle state $\left|\uparrow_{l}\right\rangle$, and the spin-down particle state $\left|\downarrow_{l}\right\rangle$. The projection of any state in the parton description onto the physical subspace with $\mathcal{K}_{l}^{\alpha}=0$ is then a superposition of $\left|\times_{l}\right\rangle,\left|\uparrow_{l}\right\rangle$, and $\left|\downarrow_{l}\right\rangle$. In terms of the parton operators, these three physical states are given by [see Eq. (18) in Ref. 23]

$$
\begin{aligned}
\left|\times_{l}\right\rangle & =\frac{1}{\sqrt{2}}\left(\mathbf{b}_{l, 1}^{\dagger}+\mathbf{b}_{l, 2}^{\dagger} \mathbf{f}_{l, \uparrow}^{\dagger} \mathbf{f}_{l, \downarrow}^{\dagger}\right)\left|\mathbf{0}_{l}\right\rangle, \\
\left|\uparrow_{l}\right\rangle & =\mathbf{c}_{l, \uparrow}^{\dagger}\left|\times_{l}\right\rangle=\mathbf{f}_{l, \uparrow}^{\dagger}\left|\mathbf{0}_{l}\right\rangle, \\
\left|\downarrow_{l}\right\rangle & =\mathbf{c}_{l, \downarrow}^{\dagger}\left|\times_{l}\right\rangle=\mathbf{f}_{l, \downarrow}^{\dagger}\left|\mathbf{0}_{l}\right\rangle,
\end{aligned}
$$

where $\left|\mathbf{0}_{l}\right\rangle$ is the vacuum of the parton operators that is defined by $\mathbf{f}_{l, \uparrow}\left|\mathbf{0}_{l}\right\rangle=\mathbf{f}_{l, \downarrow}\left|\mathbf{0}_{l}\right\rangle=0$ and $\mathbf{b}_{l, 1}\left|\mathbf{0}_{l}\right\rangle=\mathbf{b}_{l, 2}\left|\mathbf{0}_{l}\right\rangle=0$. Note that these three states are indeed physical because they satisfy $\mathcal{K}_{l}^{\alpha}\left|\times_{l}\right\rangle=\mathcal{K}_{l}^{\alpha}\left|\uparrow_{l}\right\rangle=\mathcal{K}_{l}^{\alpha}\left|\downarrow_{l}\right\rangle=0$ for all $\alpha=\{x, y, z\}$.

Before investigating the mean-field treatment, we consider a single stationary hole and aim to make a connection between its parton description and its internal quantum numbers $h, q$, and $p$. In the isolated dimer limit, there is an effective twosite system around the hole consisting of the hole site $l$ and the neighboring site $l^{\prime}=z(l)$. We assume $l \in A$ without loss of generality. Since there is a hole at site $l$ and there is no hole at site $l^{\prime}$, one holon is excited at site $l$ and no holon is excited at site $l^{\prime}$. Due to the four spinons at sites $l$ and $l^{\prime}$ that are either excited or not and the two holons at site $l$ from which exactly one is excited, the Hilbert space of the two-site system in the parton description is then 32 dimensional. However, the physical Hilbert space of the two-site system is only 2 dimensional because it is spanned by the two physical states $\left|x_{l}\right\rangle \otimes\left|\uparrow_{l^{\prime}}\right\rangle$ and $\left|x_{l}\right\rangle \otimes\left|\downarrow_{l^{\prime}}\right\rangle$. This means that the projection of any state in the parton description onto the subspace with $\mathcal{K}_{l}^{\alpha}=\mathcal{K}_{l^{\prime}}^{\alpha}=0$ is a superposition of these two physical states. Since the effective Hamiltonian of the two-site system is $H=-b_{l}^{z} b_{l^{\prime}}^{z} c_{l} c_{l^{\prime}}$, its ground state has expectation values $\left\langle i b_{l}^{z} b_{l^{\prime}}^{z}\right\rangle=\left\langle-i c_{l} c_{l^{\prime}}\right\rangle= \pm 1$. In fact, there are 16 such ground states in the parton description that take the form

$$
\begin{aligned}
\left|\Psi_{\zeta, \pm}^{r_{1}, r_{2}}\right\rangle= & \left(1 \mp i \mathbf{f}_{l, \uparrow}^{\dagger} \mathbf{f}_{l^{\prime}, \uparrow}^{\dagger}\right)\left(\mathbf{f}_{l, \downarrow}^{\dagger}\right)^{r_{1}}\left(\mathbf{f}_{l^{\prime}, \downarrow}^{\dagger}\right)^{r_{2}} \mathbf{b}_{l, \zeta}^{\dagger}\left(\left|\mathbf{0}_{l}\right\rangle \otimes\left|\mathbf{0}_{l^{\prime}}\right\rangle\right) \\
= & {\left[\left(\mathbf{f}_{l, \downarrow}^{\dagger}\right)^{r_{1}} \mathbf{b}_{l, \zeta}^{\dagger}\left|\mathbf{0}_{l}\right\rangle\right] \otimes\left[\left(\mathbf{f}_{l^{\prime}, \downarrow}^{\dagger}\right)^{r_{2}}\left|\mathbf{0}_{l^{\prime}}\right\rangle\right] } \\
& \mp i\left[\mathbf{f}_{l, \uparrow}^{\dagger}\left(-\mathbf{f}_{l, \downarrow}^{\dagger}\right)^{r_{1}} \mathbf{b}_{l, \zeta}^{\dagger}\left|\mathbf{0}_{l}\right\rangle\right] \otimes\left[\mathbf{f}_{l^{\prime}, \uparrow}^{\dagger}\left(\mathbf{f}_{l^{\prime}, \downarrow}^{\dagger}\right)^{r_{2}}\left|\mathbf{0}_{l^{\prime}}\right\rangle\right],
\end{aligned}
$$

where $\zeta=\{1,2\}$ and $r_{1,2}=\{0,1\}$. On the other hand, the projection of the ground state $\left|\Psi_{\zeta, \pm}^{r_{1}, r_{2}}\right\rangle$ onto the subspace with $\mathcal{K}_{l}^{\alpha}=\mathcal{K}_{l^{\prime}}^{\alpha}=0$ is non-zero only if the overlap of $\left|\Psi_{\zeta, \pm}^{r_{1}, r_{2}}\right\rangle$ is non-zero with either of the two physical states $\left|\times_{l}\right\rangle \otimes\left|\uparrow \iota_{l^{\prime}}\right\rangle$ or $\left|\times_{l}\right\rangle \otimes\left|\downarrow_{l^{\prime}}\right\rangle$. For $\zeta=1$, we must choose $r_{1}=0$ and $r_{2}=1$, in which case the first term in Eq. (71) has a non-zero overlap with $\left|\times_{l}\right\rangle \otimes\left|\downarrow_{l^{\prime}}\right\rangle$. For $\zeta=2$, we must choose $r_{1}=1$ and $r_{2}=0$, in which case the second term in Eq. (71) has a nonzero overlap with $\left|\times_{l}\right\rangle \otimes\left|\uparrow_{l^{\prime}}\right\rangle$. This means that the choice of exciting either $\mathbf{b}_{l, 1}^{\dagger}$ or $\mathbf{b}_{l, 2}^{\dagger}$ at the hole site $l$ before the projection determines the local magnetization at the neighboring site $l^{\prime}=z(l)$ after the projection. We therefore conclude that these two different choices correspond to different plaquette quantum numbers $p=\{0,1\}$.

Since the two-site system around the hole has only two physical states that are distinguished by the plaquette quantum number $p$, the remaining quantum numbers $h$ and $q$ are necessarily determined by the spinons around the hole site. In the regime of slow hopping, the definitions of these quantum numbers in Sec. VB are straightforward to express in terms of the Majorana fermions $b_{l}^{\alpha}$ and $c_{l}$, or equivalently, in terms of the spinon operators $\mathbf{f}_{l, \uparrow}^{(\dagger)}$ and $\mathbf{f}_{l, \downarrow}^{(\dagger)}$. Beyond the regime of slow hopping, the exact expressions become more complicated, but the general principle remains the same. For our purposes, it is enough to establish an intuitive picture from the general principle by using the interpretation in which the various hole types with different quantum numbers $h$ and $q$ have different kinds of elementary excitations bound to them. This interpretation has a simple translation in the parton description: some of the spinons around the hole site are bound to the holon at the hole site, and the quantum numbers $h$ and $q$ are in turn determined by the structure of these bound spinons.

\section{B. Mean-field treatment of the model}

In the mean-field treatment of Ref. 23, the Hamiltonian of the model is first expressed in terms of the parton operators and then subjected to an appropriate mean-field decomposition. As a result of this treatment, the mean-field Hamiltonian of the model with a small density $\rho \ll 1$ of mobile holes takes the form [see Eqs. (24) and (25) in Ref. 23] 


$$
\begin{aligned}
\tilde{H}= & \sum_{l \in A} \sum_{\alpha=x, y, z}\left\{\left[J_{\alpha} u_{\alpha}^{\alpha}-\frac{t}{4} \sum_{\zeta=1}^{2}\left(w_{\alpha}^{\zeta}+\text { c.c. }\right)\right] i c_{l} c_{\alpha(l)}-\sum_{\alpha^{\prime}=x, y, z}\left[J_{\alpha} \delta_{\alpha, \alpha^{\prime}} v_{\alpha}+\frac{t}{4} \sum_{\zeta=1}^{2}\left(w_{\alpha}^{\zeta}+\text { c.c. }\right)\right] i b_{l}^{\alpha^{\prime}} b_{\alpha(l)}^{\alpha^{\prime}}\right. \\
& \left.+\frac{t}{4}\left[v_{\alpha}-\sum_{\alpha^{\prime}=x, y, z} u_{\alpha}^{\alpha^{\prime}}\right] \sum_{\zeta=1}^{2}\left[i \mathbf{b}_{l, \zeta}^{\dagger} \mathbf{b}_{\alpha(l), \zeta}+\text { H.c. }\right]\right\}+\sum_{l} \sum_{\alpha=x, y, z} \tilde{\beta}_{l}^{\alpha} \mathcal{K}_{l}^{\alpha}-\tilde{\mu} \sum_{l} \sum_{\zeta=1}^{2} \mathbf{b}_{l, \zeta}^{\dagger} \mathbf{b}_{l, \zeta},
\end{aligned}
$$

where $u_{\alpha}^{\alpha^{\prime}}, v_{\alpha}$, and $w_{\alpha}^{\zeta}$ are the respective expectation values of the generalized bond fermion operators $\hat{u}_{\alpha}^{\alpha^{\prime}} \equiv i b_{l}^{\alpha^{\prime}} b_{\alpha(l)}^{\alpha^{\prime}}$, the generalized matter fermion operators $\hat{v}_{\alpha} \equiv-i c_{l} c_{\alpha(l)}$, and the holon coherence operators $\hat{w}_{\alpha}^{\zeta} \equiv i \mathbf{b}_{l, \zeta}^{\dagger} \mathbf{b}_{\alpha(l), \zeta}$. The constraint $\sum_{l} \sum_{\zeta}\left\langle\mathbf{b}_{l, \zeta}^{\dagger} \mathbf{b}_{l, \zeta}\right\rangle=2 N \rho$ for the total number of holes is enforced by the chemical potential $\tilde{\mu}$, while the softened gauge constraint $\left\langle\mathcal{K}_{l}^{\alpha}\right\rangle=0$ is enforced by the Lagrange multiplier $\tilde{\beta}_{l}^{\alpha}$ for all $l$ and $\alpha$. Importantly, the mean-field Hamiltonian in Eq. (72) is an extension of that in Ref. 23. It is applicable to the gapped phase of the model, where the coupling strengths $J_{\alpha}$ are different: $J_{z}=1$ and $J \equiv J_{x}=J_{y} \ll 1$.

The mean-field Hamiltonian in Eq. (72) can be solved by a self-consistent procedure in terms of the expectation values $u_{\alpha}^{\alpha^{\prime}}, v_{\alpha}$, and $w_{\alpha}^{\zeta}$. In the absence of holes $(\rho=0)$, there is a coupling of strength $J_{\alpha}$ between the bond fermion expectation value $u_{\alpha}^{\alpha}$ and the matter fermion expectation value $v_{\alpha}$ along each bond of $\alpha$ type. Keeping only the lowest-order terms in $J \ll 1$, the self-consistent solutions for these expectation values are $u_{\alpha}^{\alpha}=v_{z}=1$ and $v_{x}=v_{y}=J / 2$. Note that the same expectation values are obtained from the exact solution of the model in Sec. IIII In the presence of holes $(\rho>0)$, the holons all condense into their lowest-energy state at zero momentum, and hence the holon coherence expectation values are $w_{\alpha}^{\zeta} \sim \rho$. This means that the original terms $J_{\alpha} u_{\alpha}^{\alpha}$ and $J_{\alpha} \delta_{\alpha, \alpha^{\prime}} v_{\alpha}$ in the first two square brackets of Eq. (72) are in competition with new terms on the order of $t \rho$. The expectation values $u_{\alpha}^{\alpha}$ and $v_{\alpha}$ for $\rho>0$ are then close to those for $\rho=0$ as long as these new terms are negligible with respect to the original terms. In particular, the bond fermion expectation values $u_{x}^{x}$ and $u_{y}^{y}$ remain close to 1 as long as $t \rho \ll J_{x, y} v_{x, y} \sim J^{2}$, while the bond fermion expectation value $u_{z}^{z}$ and the matter fermion expectation value $v_{z}$ remain close to 1 as long as $t \rho \ll J_{z} v_{z}, J_{z} u_{z}^{z} \sim 1$.

\section{Discussion of ground-state properties}

We are now ready to make a comparison between the meanfield ground state obtained from Eq. (72) and the exact ground state discussed in Secs. VIIA and VIII Although there are general trends in the phase diagram of the model that are common to both approaches, this comparison reveals several interesting discrepancies between the exact description and the mean-field treatment. In particular, there are two significant discrepancies concerning the internal degrees of freedom and the particle statistics of mobile holes.
The most important result of our exact study is that each hole has three internal degrees of freedom and that it can be characterized by three corresponding quantum numbers $h, q$, and $p$. The quantum number $p$ describes a local magnetization around the hole, while the quantum numbers $h$ and $q$ capture the possibility of an elementary excitation (flux or fermion) being bound to it. The parton description in Ref. 23 incorporates the quantum number $p$ via the introduction of two distinct holon species (see Sec. IXA). However, the mean-field treatment is unable to represent the quantum numbers $h$ and $q$ : it ignores the possibility of bound states between holes and elementary excitations as it inherently neglects any correlations between these independent degrees of freedom.

In the regime of slow hopping, it is straightforward to verify explicitly that all holes in the mean-field ground state have quantum numbers $h=0$ and $q=0$ as they have no elementary excitations bound to them. Since $t \ll J^{4}$ and $\rho \ll 1$ in this regime, the conditions $t \rho \ll J^{2}$ and $t \rho \ll 1$ are both satisfied, and hence the mean-field expectation values $u_{\alpha}^{\alpha}$ and $v_{\alpha}$ for $\rho>0$ are close to those at $\rho=0$. On the other hand, these expectation values are the same as those obtained from the exact solution of the undoped model. Since the exact ground state of the undoped model is free of elementary excitations by definition, the mean-field ground state of the doped model has no elementary excitations either. Note that the quantum numbers of the mean-field ground state are then consistent with those of the exact ground state in the case of $J^{8} \ll t \ll J^{4}$ but not in the case of $t \ll J^{8}$ (see Sec. VIIA).

The particle statistics of the various hole types are further important results of our exact study. Unsurprisingly, the particle statistics depends on the quantum numbers $h$ and $q$ as the binding of an elementary excitation can lead to a statistical transmutation. Since only bare holes with $h=0$ and $q=0$ are captured by the mean-field treatment, the relevant comparison is between the bare holes of the exact description and the holons of the mean-field treatment. We find a remarkable discrepancy in this respect: the bare holes of our exact study are fermions, while the holons of the parton description in Ref. 23 are bosons. It would then be interesting to resolve this discrepancy by considering a fermionic analogue of the mean-field treatment in Ref. 23. For example, an appropriate transformation between spinful bosons and spinful fermions ${ }^{28}$ could be used to relate the two species of bosonic holons and the fermionic bare holes with $p=\{0,1\}$. Alternatively, it is natural to ask how an analysis going beyond the mean-field saddle point could provide the correct statistics.

Beyond the regime of slow hopping, we can compare the evolution of the mean-field ground state as a function of $t, J$, 
and $\rho$ with our picture of the exact ground state where holes are surrounded by clouds of fluctuating excitations. In the mean-field treatment, there are two important characteristic scales of $t \rho$. First, the bond fermion expectation values $u_{x}^{x}$, $u_{y}^{y}$, and $u_{z}^{z}$ are all close to 1 only for $t \rho \ll J^{2}$, and flux excitations then start appearing at $(t \rho)_{P} \sim J^{2}$. Second, the matter fermion expectation values $v_{z}$ are close to 1 only for $t \rho \ll 1$, and fermion excitations then start appearing at $(t \rho)_{f} \sim 1$. In the language of the exact description in Sec.VIII the critical value $(t \rho)_{P, f}$ corresponds to the critical density at which the fluctuating fluxes (fermions) around different holes merge. If we assume that our upper bounds on the excitation cloud radii are good estimates so that $R_{P} \sim \sqrt{t / J^{4}}$ and $R_{f} \sim \sqrt{t}$, the corresponding critical values from Sec. VIII are $(t \rho)_{P} \sim J^{4}$ and $(t \rho)_{f} \sim 1$. These results have a simple interpretation: each kind of excitation starts appearing when the kinetic energy density $t \rho$ reaches its excitation energy. However, by using this interpretation, we obtain inconsistent values for the flux excitation energy as it is $E_{P} \sim J^{4}$ in the exact description and $E_{P} \sim J^{2}$ in the mean-field treatment. The reason for this inconsistency is that flux excitations do not appear explicitly in the mean-field treatment but instead are decoupled as independent bond fermion excitations.

At the isotropic point of $J=1$, for which the mean-field theory in Ref. 23 is devised, the regime of slow hopping is unattainable for any hopping amplitude due to the existence of gapless fermionic excitations. It is then not a priori possible to think of each hole as possessing well-defined internal degrees of freedom. ${ }^{15}$ The innocuous choice of a quasiparticle representing the hole can be non-obvious due to the intricate many-body problem posed by the detailed hole dynamics, and in the most extreme scenario, it can even become ill-defined as the coupling between the hole and the gapless excitations renders the quasiparticle description problematic in itself. It is a natural extension of our present work to consider a single isolated hole in the gapless phase of the model and discuss its potential quasiparticle representation along with any internal degrees of freedom possessed by it.

\section{SUMMARY AND OUTLOOK}

In this work, we presented a thorough and controlled microscopic study of slow mobile holes hopping in the spatially anisotropic (Abelian) gapped phase of the Kitaev honeycomb model. We found that the mobile holes in the model have internal degrees of freedom as they can bind the fractional excitations of the model and that the resulting hole types with different fractional excitations bound to them are fundamentally different in terms of their single-particle and multi-particle properties. We now conclude the paper with two suggestions for the future direction of this research.

The interest in doped topological states is in part due to their identification as possible candidates for high-temperature superconductors. ${ }^{2,3}$ If Cooper pairs are formed by extra electrons or missing electrons (holes) in such a doped topological state, the condensation of these Cooper pairs can lead to superconducting behavior. As discussed briefly in Sec. VC the holes in the Kitaev honeycomb model form bound pairs if the Coulomb repulsion is strong enough to counteract phase separation but not strong enough to counteract pair formation. It is then natural to ask what kind of internal degrees of freedom these hole pairs possess and what their manifestations are in the superconducting behavior of hole pairs. Furthermore, the question of superconductivity is of central importance in the complementary mean-field works. ${ }^{22,23}$ Consequently, an exact study of hole pairs could further clarify the relation between the exact description and the mean-field treatments.

The binding of fractional excitations by mobile holes is interesting in part because it provides a controlled way of introducing fractional particles into the model and manipulating the resulting quantum state by exploiting the anyonic statistics of these fractional particles. ${ }^{27}$ Importantly, the Kitaev honeycomb model has even more exotic fractional excitations in its spatially isotropic (non-Abelian) gapped phase. ${ }^{13}$ It is then natural to expect that these fractional excitations with non-Abelian anyonic statistics can also be bound to mobile holes and that the properties of the resulting fractional particles would be interesting to explore.

\section{Acknowledgments}

We thank F. J. Burnell, I. Kimchi, C. R. Laumann, and S. H. Simon for enlightening discussions. This work was supported by the EPSRC under Grant No. EP/I032487/1, and by the Helmholtz Virtual Institute "New States of Matter and Their Excitations".
${ }^{1}$ E. Dagotto, Rev. Mod. Phys. 66, 763 (1994).

2 P. A. Lee, N. Nagaosa, and X.-G. Wen, Rev. Mod. Phys. 78, 17 (2006).

${ }^{3} \mathrm{P} . \mathrm{W}$. Anderson, The Theory of Superconductivity in the High-T $T_{C}$ Cuprates (Princeton University Press, Princeton, 1997).

4 J. G. Bednorz and K. A. Müller, Z. Phys. B: Condens. Matter 64, 189 (1986).

5 G. Misguich and C. Lhuillier, arXiv:cond-mat/0310405.

${ }^{6}$ P. W. Anderson, Science 235, 1196 (1987).

7 P. W. Anderson, Mater. Res. Bull. 8, 153 (1973).

${ }^{8}$ R. Moessner and K. S. Raman, arXiv:0809.3051
${ }^{9}$ S. A. Kivelson, D. S. Rokhsar, and J. P. Sethna, Phys. Rev. B 35, 8865(R) (1987).

10 D. S. Rokhsar and S. A. Kivelson, Phys. Rev. Lett. 61, 2376 (1988); R. Moessner and S. L. Sondhi, Phys. Rev. Lett. 86, 1881 (2001).

11 R. Rajaraman, arXiv:cond-mat/0103366

12 X. G. Wen and Q. Niu, Phys. Rev. B 41, 9377 (1990).

13 A. Y. Kitaev, Ann. Phys. 321, 2 (2006).

14 A. J. Willans, J. T. Chalker, and R. Moessner, Phys. Rev. Lett. 104, 237203 (2010); A. J. Willans, J. T. Chalker, and R. Moessner, Phys. Rev. B 84, 115146 (2011). 
15 F. Trousselet, P. Horsch, A. M. Oleś, and W.-L. You, Phys. Rev. B 90, 024404 (2014).

${ }^{16}$ G. Baskaran, Z. Zou, and P. W. Anderson, Solid State Commun. 88, 853 (1993).

17 M. C. Gutzwiller, Phys. Rev. Lett. 10, 159 (1963); T. A. Kaplan, P. Horsch, and P. Fulde, Phys. Rev. Lett. 49, 889 (1982).

18 X.-G. Wen, Phys. Rev. B 65, 165113 (2002).

${ }^{19}$ G. Baskaran and P. W. Anderson, Phys. Rev. B 37, 580(R) (1988).

20 A. M. Essin and M. Hermele, Phys. Rev. B 87, 104406 (2013).

${ }^{21}$ M. Hermele, T. Senthil, M. P. A. Fisher, P. A. Lee, N. Nagaosa, and X.-G. Wen, Phys. Rev. B 70, 214437 (2004).

${ }^{22}$ F. J. Burnell and C. Nayak, Phys. Rev. B 84, 125125 (2011); T. Hyart, A. R. Wright, G. Khaliullin, and B. Rosenow, Phys. Rev. B 85, 140510(R) (2012); J.-W. Mei, Phys. Rev. Lett. 108, 227207
(2012); R. Schaffer, S. Bhattacharjee, and Y. B. Kim, Phys. Rev. B 86, 224417 (2012); S. Okamoto, Phys. Rev. B 87, 064508 (2013).

23 Y.-Z. You, I. Kimchi, and A. Vishwanath, Phys. Rev. B 86, 085145 (2012).

${ }^{24}$ S. Kivelson, Phys. Rev. B 39, 259 (1989); D. Poilblanc, Phys. Rev. Lett. 100, 157206 (2008).

25 H. Yao, S.-C. Zhang, and S. A. Kivelson, Phys. Rev. Lett. 102, 217202 (2009).

${ }^{26}$ E. H. Lieb, Phys. Rev. Lett. 73, 2158 (1994).

${ }^{27}$ S. Dusuel, K. P. Schmidt, and J. Vidal, Phys. Rev. Lett. 100, 177204 (2008).

${ }^{28}$ L. Balents, M. P. A. Fisher, and C. Nayak, Phys. Rev. B 61, 6307 (2000). 